\title{
AAV-based gene therapy for axonal regeneration in a rat model of rubrospinal tract lesion
}

\author{
Dissertation \\ For the award of the degree \\ "Doctor of Philosophy" \\ Division of Mathematics and Natural Sciences \\ of the Georg-August-Universität-Göttingen, \\ Germany
}

Submitted by

Malleswari Challagundla

Born in

Kothaganeshunipadu (India)

Göttingen, February 2014 
Prof. Dr. Mathias Bähr

Prof. Dr. André Fischer

Prof. Dr. Wolfgang Brück

\section{Extended thesis committee members}

Prof. Dr. Tiago Fleming Outeiro

Dr. Camin Dean

Prof. Dr. Ralf Heinrich 


\section{Declaration}

I hereby declare that the thesis "AAV-based gene therapy for axonal regeneration in a rat model of rubrospinal tract lesion" has been written independently with no other sources and aids otherwise quoted.

Malleswari Challagundla, Göttingen, February 2014. 


\section{TABLE OF CONTENTS}

Declaration $\quad 3$

TABLE OF CONTENTS

1 Introduction $\quad 7$

$\begin{array}{ll}1.1 \text { Spinal cord injury } & 7\end{array}$

$\begin{array}{ll}\text { 1.1.1 Spinal cord anatomy } & 7\end{array}$

1.1.2 Corticospinal and rubrospinal tracts $\quad 8$

$\begin{array}{lr}\text { 1.1.3 Spinal cord lesion and pathophysiology } & 10\end{array}$

$\begin{array}{ll}1.2 & \text { Axonal regeneration and plasticity after SCI } \\ 12\end{array}$

$\begin{array}{ll}\text { 1.3 Barriers of axonal regeneration in the CNS } & 13\end{array}$

$\begin{array}{ll}\text { 1.3.1 Myelin inhibitors } & 13\end{array}$

$\begin{array}{ll}\text { 1.3.2 Scar tissue } & 13\end{array}$

1.4 Current treatment approaches for SCI $\quad 14$

1.5 Anti-scarring treatment (BPY-DCA) 16

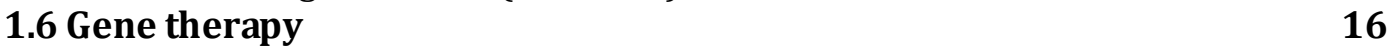

$\begin{array}{ll}\text { 1.6.1 Rho and Rho kinase (ROCK) } & 17\end{array}$

$\begin{array}{ll}\text { 1.6.2 BAG1 } & 20\end{array}$

$\begin{array}{ll}1.6 .3 \text { microRNA-134 } & 21\end{array}$

1.6.4 Reggie1 22

1.7 Aims of the thesis $\quad 23$

2 Materials $\quad 24$

2.1 Instruments $\quad 24$

2.2 Chemicals and Consumables $\quad 25$

2.3 Solutions and Buffers $\quad 26$

2.3.1 Mowiol $\quad 26$

2.3.2 Paraformaldehyde (PFA) 4\% 26

2.3.3 1x PBS 26

$2.3 .410 \%, 20 \%, 30 \%$ of sucrose (sucrose for cryoprotection) 26

2.3.5 10\% Normal Goat Serum (NGS) 27

$\begin{array}{ll}\text { 2.3.6 Citrate buffer (antigen retrieval solution) } & 27\end{array}$

$\begin{array}{ll}\text { 2.3.7 Blocking solution for immunohistochemistry } & 27\end{array}$

2.3.8 TRIS buffer, $20 \mathrm{mM}$, pH $8.1 \quad 27$

2.3.9 BPY-DCA (2,2'-bipyridine-5, 5'-dicarboxylic acid), 40 mM, pH 7.4 27

$\begin{array}{ll}2.4 \text { Primary Antibodies } & 28\end{array}$

$\begin{array}{ll}2.5 \text { Secondary Antibodies } & 28\end{array}$

2.6 Anterograde tracing dyes $\quad 29$

3 Methods $\quad 30$

3.1 Animals $\quad 30$

$\begin{array}{ll}3.2 \text { Cloning of viral vectors } & \mathbf{3 0}\end{array}$

3.3 Experimental set-up and animal groups $\mathbf{3 2}$

3.3.1 Pilot project: Effects of the combination of BAG1 overexpression and antiscarring treatment with BPY-DCA in a rat model of dorsal spinal cord hemisection.

3.3.2 Anterograde tracing of RST before and after dorsal spinal cord hemisection

3.3.3 Effects of AAV.BAG1, AAV.reggie1, AAV.miRNA-134 and AAV.sh.ROCK2 on RST in a rat model of dorsal spinal cord hemisection 36

$\begin{array}{ll}3.4 \text { Animal surgeries } & 37\end{array}$ 
3.4.1 Stereotactic injection of AAV into the brain

3.4.2 Right dorsal hemisection of the spinal cord

3.4.3 Osmotic mini-pump implantation for the anti-scarring treatment delivery to

$\begin{array}{ll}\text { the spinal cord after dorsal hemisection } & 40\end{array}$

3.4.4 Pre- and post-operative care of the animals 43

3.4.5 Perfusions and tissue processing 43

3.5 Histology

$\begin{array}{ll}3.5 .1 \text { Cryosectioning } & 44\end{array}$

3.5.2 Immunohistochemistry $\quad 45$

$\begin{array}{ll}\text { 3.6 Data analysis and quantifications } & \mathbf{4 6}\end{array}$

3.6.1 NeuN counting for RN cell survival quantification $\quad 46$

$\begin{array}{ll}\text { 3.6.2 Quantification of axonal sprouting } & 46\end{array}$

3.6.3 Quantification of lesion size and axonal degeneration 47

$\begin{array}{ll}\text { 3.7 Behavioral studies } & \mathbf{4 8}\end{array}$

$\begin{array}{ll}\text { 3.7.1 BBB - open field test } & 48\end{array}$

$\begin{array}{ll}\text { 3.7.2 Horizontal ladder rung test } & 49\end{array}$

$\begin{array}{ll}\text { 3.7.3 CatWalk } & \text { TM gait analysis } \\ \end{array}$

$\begin{array}{ll}3.8 \text { Statistics } & 51\end{array}$

4 Results $\quad 52$

4.1 Pilot project: Combination treatment with AAV.BAG1 and BPY-DCA for 16 weeks after dorsal spinal cord hemisection in rats $\quad 52$

4.1.1 Viral injections and spinal cord lesion 52

4.1.2 Effect of combination treatment with AAV.BAG1 and AST on axonal sprouting at 16 weeks after dorsal spinal cord hemisection $\quad 55$

4.1.3 Osmotic mini-pump implantation resulted in increased lesion size at 16 weeks after dorsal spinal cord hemisection

4.1.4 Effect of combination treatment with AAV.BAG1 and AST on motor behavior

at 16 weeks after dorsal spinal cord hemisection. $\quad 60$

4.2 Anterograde tracing of RST after dorsal spinal cord hemisection $\quad 62$

4.2.1 Anterograde tracing of the RST with AAV.EGFP before and BDA or

AAV.DsRed after the right dorsal spinal cord hemisection 62

4.2.2 Anterograde tracing of RST immediately after dorsal spinal cord hemisection with AAV.EGFP

4.3 Gene therapy with AAV.BAG1,AAV.reggie1,AAV.miRNA-134, and

AAV.sh.ROCK2 at 12 weeks after dorsal spinal cord hemisection

4.3.1 Experimental set up for gene therapy with AAV.BAG1, AAV.reggie1, AAV.miRNA-134, and AAV.sh.ROCK2 at 12 weeks after dorsal spinal cord hemisection

4.3.2 Effect of AAV.reggie1 and AAV.miRNA-134 on the red nucleus after dorsal

spinal cord hemisection

4.3.3 AAV.BAG1 and AAV.sh.ROCK2 improve survival of RN neurons 12 weeks

after dorsal spinal cord hemisection

4.3.4 Axonal sprouting at cervical SC is enhanced by treatment with AAV. BAG1 and AAV.sh.ROCK2

4.3.5 Axonal sprouting at thoracic SC is enhanced by treatment with AAV. BAG1 and AAV.sh.ROCK2

4.3.6 Effects of AAV.BAG1 and AAV.sh.ROCK2 on lesion size and axonal degeneration

4.3.7 Effect of AAV.BAG1 and AAV.sh.ROCK2 on motor recovery 83

\section{Discussion}

5.1 Effects of a combination therapy with AAV.BAG1 and AST at 16 weeks after dorsal spinal cord hemisection 
5.1.1 Effects of anti-scarring treatment

5.1.2 Effects of osmotic mini-pump implantation on lesion size 87

5.2 Optimization of anterograde RST tracing

5.3 Effects of gene therapy with AAV.BAG1 and AAV.reggie1,AAV.miRNA-134 and ROCK2.sh.RNA

5.3.1 Toxic effects of AAV.miRNA-134 and AAV.reggie1 expression $\quad 89$

5.3.2 Effects of AAV.BAG1 and AAV.sh.ROCK2 on RN neuronal survival 90

5.3.3 Effects of AAV.BAG1 and AAV.sh.ROCK2 on axonal sprouting and axonal regeneration

5.3.4 Effects of AAV.BAG1 and AAV.sh.ROCK2 on axonal degeneration and lesion size

5.3.5 Effects of AAV.BAG1 and AAV.sh.ROCK2 on motor recovery 92

$\begin{array}{ll}5.4 \text { Conclusion and perspectives } & 93\end{array}$

6 Summary and conclusions $\quad 94$

$\begin{array}{ll}7 \text { References } & 96\end{array}$

8 Annexes $\quad 110$

$\begin{array}{lr}\text { List of Abbreviations } & 110\end{array}$

$\begin{array}{lr}\text { Supplementary images } & 113\end{array}$

$\begin{array}{lr}\text { Acknowledgements } & 116\end{array}$

Curriculum vitae Fehler! Textmarke nicht definiert. 


\section{Introduction}

\subsection{Spinal cord injury}

\subsubsection{Spinal cord anatomy}

The brain and spinal cord are referred to as the central nervous system (CNS). The Spinal cord descends down from the brain until the lower back and is protected by bony vertebral columns. It is surrounded by a clear fluid called, cerebrospinal fluid (CSF). CSF acts like a cushion and protects the delicate spinal tissue from damage, which may occur due to banging against bony vertebrae. The vertebral column and spinal canal in humans is divided into 5 segments, namely 7 cervical (C), 12 thoracic (Th), 5 lumbar (L), 5 sacral (S) and 1 coccygeal (Co) segments. The Co segment is mainly vestigial in humans. In rats the spinal cord is made up of 8 cervical $(C), 13$ thoracic (Th), 6 lumbar (L), and 4 sacral (S) and 3 coccygeal (Co) segments. The spinal cord is composed of gray and white matter. The gray matter is mainly composed of neuronal cell bodies. In the transverse section of the spinal cord, the gray matter appears as butterfly or " $\mathrm{H}$ "-shaped structure. The white matter is composed mainly of axons and glial cells. Generally, a tract is defined as a group of nerve fibers of the same origin, course and termination, e.g. corticospinal tract (CST), rubrospinal tract (RST). A pathway is defined as a group of tracts with functional relation, e.g. motor pathways. The pathways carry the information to and from the brain and body organs. Based on the information they carry, they are divided into ascending and descending pathways. Tracts of the ascending pathway carry sensory information such as touch and pain from body organs to the brain, while the tracts of descending pathway send the response or modulate the information to motor action from brain to other parts of the body (Anderson et al., 2009). Figure 1 shows ascending and descending tracts of the rat and human cervical spinal cord. 
a

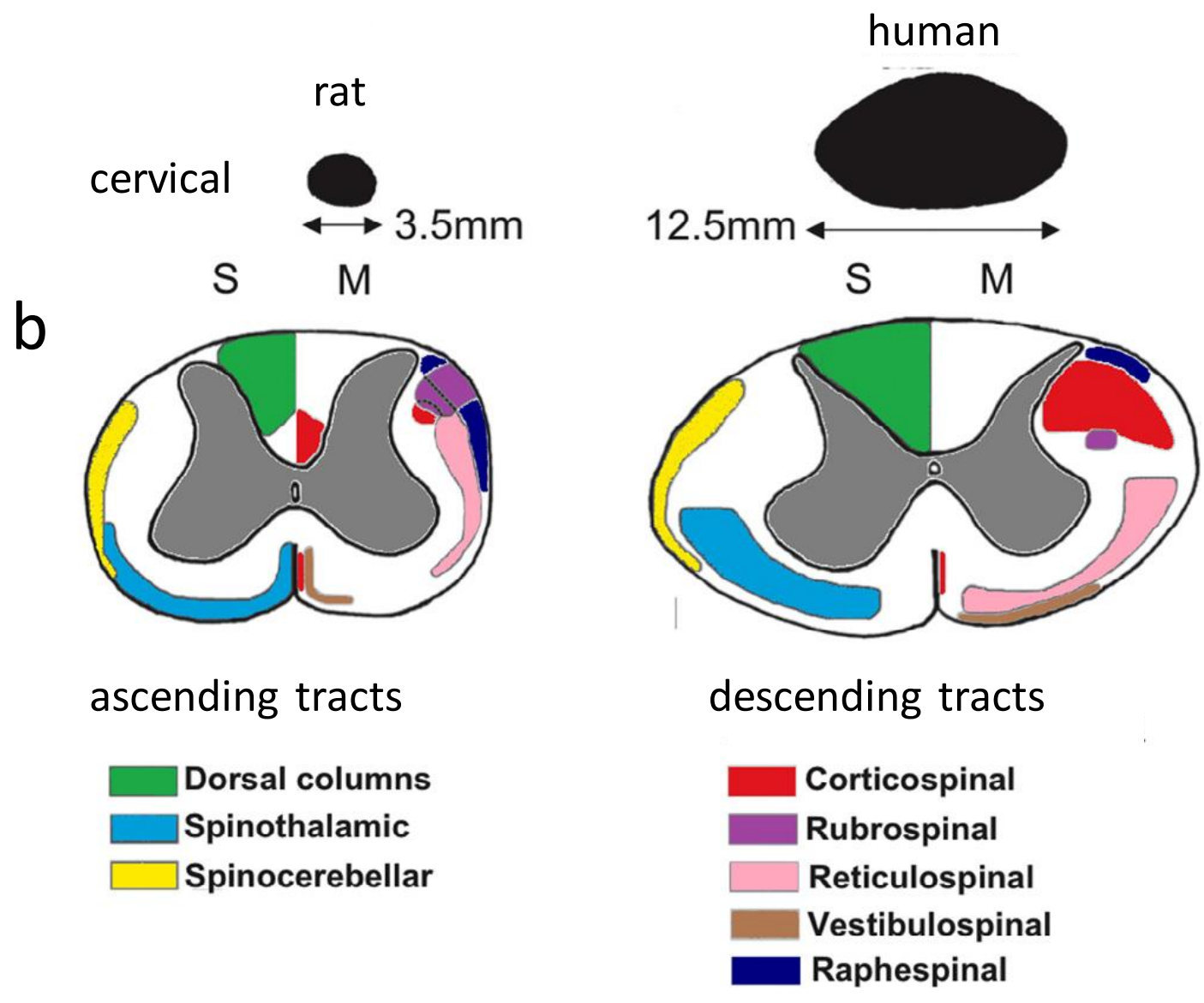

Figure 1. Anatomy of the rat and human spinal cord transverse section. (a). Comparative cross-section size of the rat and human cervical spinal cord. (b). Schematic diagram of the major ascending and descending tracts in the rat and human spinal cord. $S=$ sensory tracts; $M=$ motor tracts. Image adapted from (Anderson et al., 2009; Silva et al., 2014).

\subsubsection{Corticospinal and rubrospinal tracts}

Rodent studies of axonal regeneration and plasticity in the CNS mainly focus on two descending motor tracts: the corticospinal tract (CST) and the rubrospinal tract (RST). The CST, which is the major motor tract, originates from layer $\mathrm{V}$ pyramidal cells in the primary sensorimotor cortex. The CST is located primarily at the ventromedial base of the dorsal columns in the rat spinal cord. CST projections are also seen in dorsolateral white matter and also in a small ipsilateral, ventromedial component, which comprises $10-20 \%$ of the descending fibers. CST fibers are found at all levels of the spinal cord 
in a rat, and mainly control the voluntary, fast and precise paw and finger movements, such as grasping for food. A major difference between the rat and human spinal cord is the location of the CST. In the rat the CST is located in the dorsal white matter, whereas in humans, it is located in the lateral white matter (Gemma et al., 1987; Metz and Whishaw, 2002; Schwartz et al., 2005). Figure 2 shows the anatomy of the rat CST and RST.

a

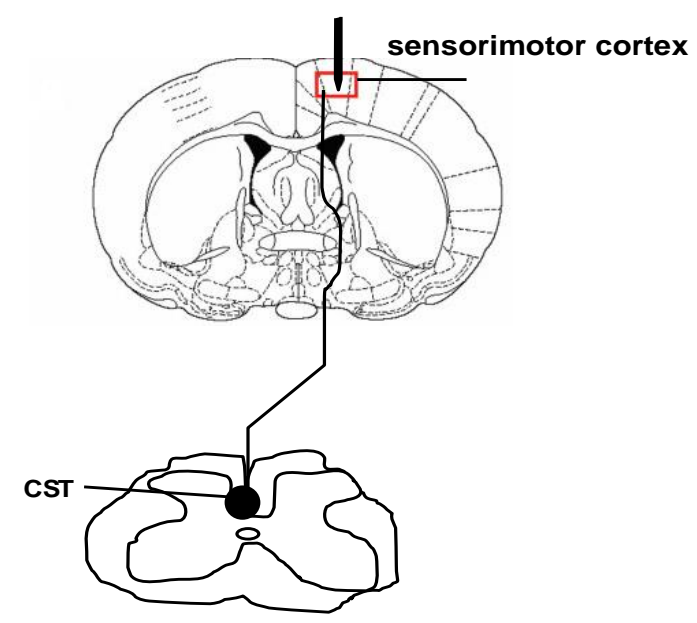

b

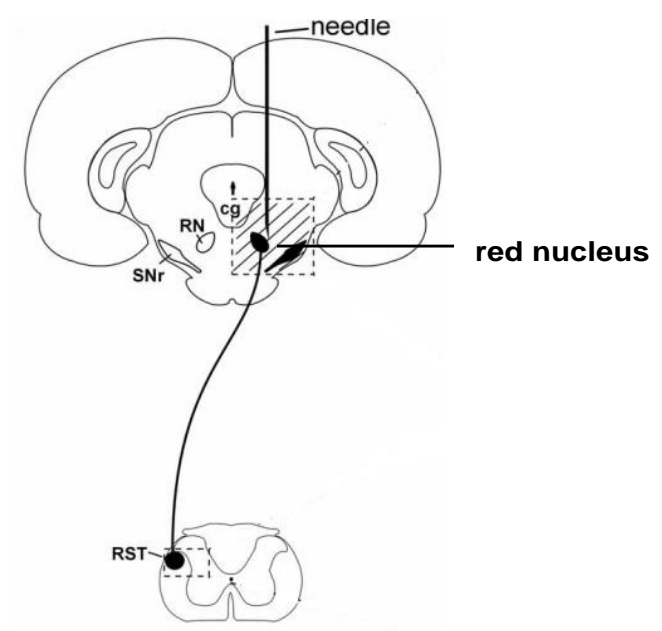

Figure 2. Anatomy of the rat corticospinal (CST) and rubrospinal tracts (RST). (a) The CST starts at the sensorimotor cortex layer $V$ of the brain and runs in the contralateral dorsal and lateral white matter of the spinal cord. (b) The RST starts at the red nucleus (RN) of the midbrain and runs in the contralateral dorsal and lateral white matter of the spinal cord. $\mathrm{SNr}=$ substantia nigra pars reticulata; $c g=$ central gray matter; Images (a) and (b) were adapted from Paxinos and Watson, 1998; Ruitenberg et al., 2002.

The RST originates from the magnocellular portion of the red nucleus (RN) of the midbrain, running through the dorsal lateral white matter of the rat spinal cord then through all levels of the spinal cord. The RST terminals are mainly distributed in Rexed's laminae V and VI as well as in the dorsal part of lamina $\mathrm{VII}$ in rat spinal cord (SC). Most rubrospinal axonal projections are found to descend contralaterally in the dorsolateral funiculus while few of the projections descend in the ipsilateral dorsolateral funiculus. Unlike the CST, the RST is entirely confined to the dorsal lateral funiculus of the rat SC and dorsal lateral hemisection can completely lesion the entire RST (Figure 1b). 
This makes the RST an excellent model lesion target for regeneration studies with partial lesions. In addition to this, the small size of the RN makes it an ideal target for therapeutic proof of concept studies (Blits et al., 2010; Brown, 1974; Koda et al., 2004; Küchler et al., 2002; Ruitenberg et al., 2002, 2004). The RST is a well-defined spinal nerve tract and its function is to control fore and hind-limb muscles, over-ground locomotion and skilled movements in rats, hence injury to the RST results in functional deficits of the afore mentioned limbs and movements (Hendriks et al., 2006; Webb and Muir, 2003).

\subsubsection{Spinal cord lesion and pathophysiology}

Spinal cord injury (SCl) is a devastating and largely irreversible clinical condition, which can lead to permanent sensory and motor functional disabilities. Worldwide, an estimated 2.5 million people suffer from $\mathrm{SCl}$ and more than 130,000 new injuries are reported annually (Kundi, 2013; Rossignol et al., 2007; Thuret et al., 2006). SCl can be categorized into (1) complete $\mathrm{SCl}$ leading to none or a few signals passing from the brain to the site of injury and, (2) incomplete lesions which, due to partial cut or crush, result in an incomplete loss of signals with low chances of partial recovery of motor or sensory function over time. Depending on the level of the $\mathrm{SCl}$ functional deficits and complications vary largely.

The pathophysiology of the human $\mathrm{SCl}$ can be described as a two-step mechanism, which includes primary and secondary lesions. Primary lesions result as a direct damage to the axons, nerve cells and blood vessels, which lead the physiological tissue deformation. However, primary lesions trigger the activation of several biochemical signalling pathways leading to secondary lesion formation (Bunge et al., 1993). The secondary lesion can be subdivided into an immediate, acute, intermediate and chronic phase (Rowland et al., 2008). The immediate phase (0-2 hrs.) includes transection of axons, death of neuronal and glial cells and spinal shock leading to instant functional loss in case of complete $\mathrm{SCl}$. Acute axonal degeneration (AAD) was reported in mice in the first hours after $\mathrm{SCl}$, which includes swelling of 
the axons and haemorrhage in the central grey matter of the SC (Hayes et al., 2007; Norenberg et al., 2004; Wang et al., 2012). The events that take place during 2 to 48 hours after $\mathrm{SCl}$ are categorized as the early acute phase. This phase is mainly characterized by several biochemical events such as oedema, inflammation, continuous haemorrhage, excitotoxicity, ionic dysregulation, free radical production, demyelination, increase in the blood brain barrier permeability and apoptotic cell death (Crowe et al., 1997; Emery et al., 1998; Mautes et al., 2000; Schnell et al., 1999; Tator and Fehlings, 1991; Tator and Koyanagi, 1997). From day 2 to the end of week 2 follows the subacute phase which shows an active phagocytic response and delayed astrocyte activation forming the glial scar, which becomes a barrier for axonal regeneration (Donnelly and Popovich, 2008; Hagg and Oudega, 2006). The intermediate phase, which lasts for 2 to 6 months, is characterised by a continuous development of the glial and fibrous scar with spontaneous regenerative axonal sprouting. In the chronic phase (6 months onwards), stabilization of the lesion, scar, cyst formation and continuation of Wallerian degeneration takes place (Coleman and Perry, 2002; Ehlers, 2004).

Understanding the molecular and cellular events that take place after the $\mathrm{SCl}$ is important for the development of animal models to target different pathophysiological phenomena that are specific to each individual phase and to eventually develop successful therapeutic strategies for SCI (Figure 3). 


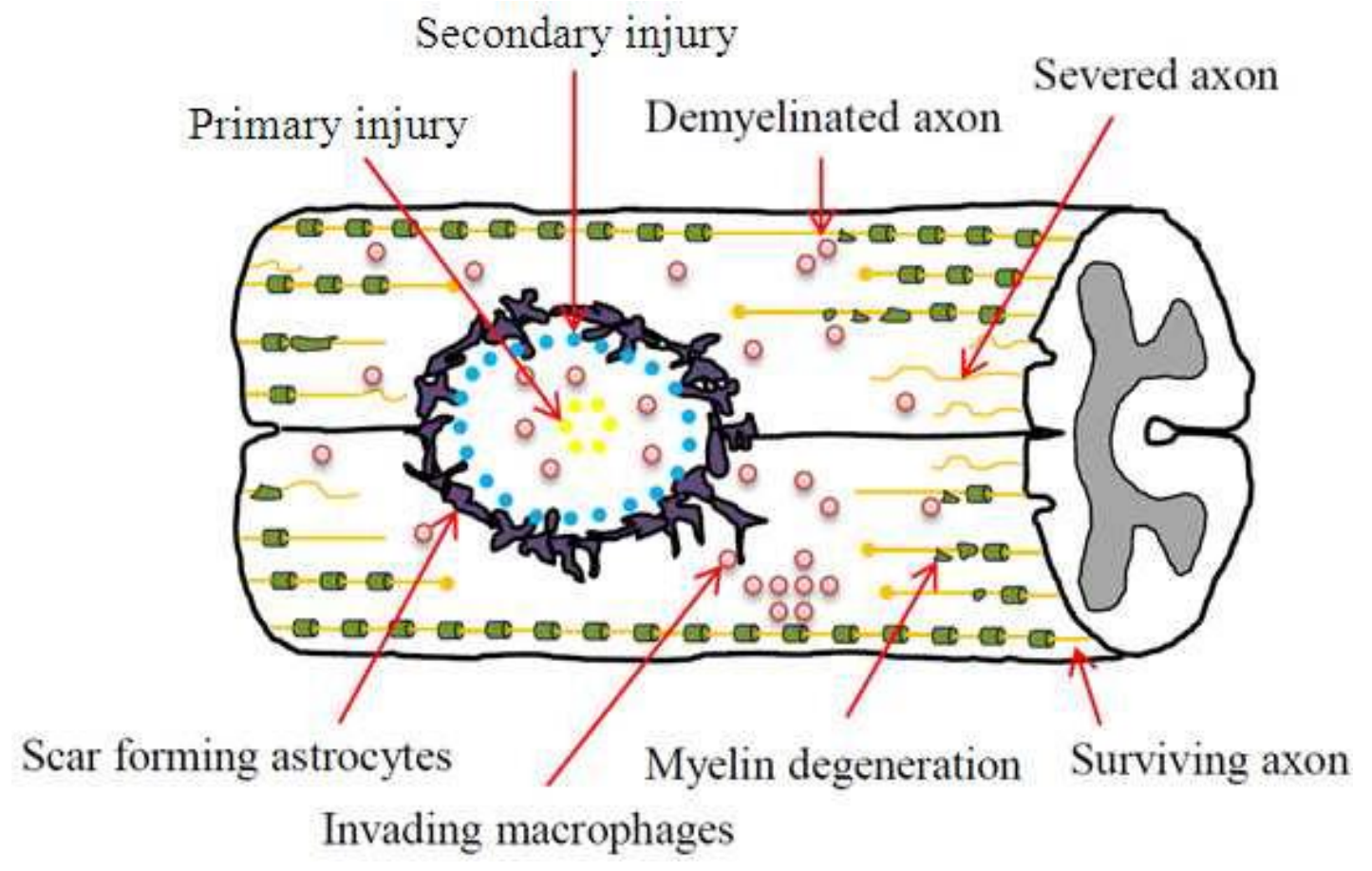

Figure 3. Schematic representation of the pathological events after SCI. Rapid degeneration of neuronal cell bodies as a result of SCl is accompanied by local bleeding at the lesion epicenter further leading to ischemia, excitotoxicity, free-radical generation, inflammation, oedema and programmed cell death, which contribute to secondary degeneration and increase in the lesion core size with time. Elevated levels of collagen and chondroitin sulphate proteoglycans (CSPG) by reactive astrocytes contribute to scar formation. Image was adapted from Kundi, 2013.

\subsection{Axonal regeneration and plasticity after $\mathrm{SCl}$}

The mammalian adult CNS is extremely limited in axonal regeneration. However, some limited spontaneous collateral axonal sprouting is observed as a compensatory mechanism by the lesioned or spared axons in partial SC lesions (Schwab, 1996; Weidner et al., 2001). Axonal sprouts were reported within 6 to 48 hours of SCl in mice (Kerschensteiner et al., 2005). In the later phases after $\mathrm{SCl}$ (2 weeks onwards) spontaneous axonal sprouting was also reported, but the extent and duration of the sprouting varies in different spinal tracts (Hagg and Oudega, 2006; Hill et al., 2001). However, spontaneous axonal sprouting is not sufficient enough to sustain functional recovery and in most cases this limited sprouting is aborted due to different inhibitory molecules and the scar tissue in the chronic stage. 


\subsection{Barriers of axonal regeneration in the CNS}

\subsubsection{Myelin inhibitors}

Research focussing on the repair of the adult CNS had extensively studied the intrinsic factors contributing to regenerative failure as well as the environment of the lesion. The myelin sheath around the axons, which is formed by oligodendrocytes and whose main function is to ensheath the healthy axon in order to permit saltatory conductivity, is a rich source of many inhibitory molecules. After lesion, severed axons are exposed to various myelin-associated inhibitory molecules such as Nogo A, myelin-associated glycoprotein (MAG) and oligodendrocyte myelin glycoprotein (OMGp) (Caroni and Schwab 1988; McKerracher, David et al. 1994; Mukhopadhyay, Doherty et al. 1994), chondroitin sulfate proteoglycans (Rolls et al., 2009; Yiu and $\mathrm{He}$, 2006), semaphorins, and ephrins (Benson et al., 2005; Goldshmit et al., 2004) which act via the activation of the GTPase RhoA. Downstream of RhoA, Rho-associated kinase (ROCK) propagates the inhibitory signal and thus plays a key role in axonal growth inhibition (Yamashita et al., 2002).

\subsubsection{Scar tissue}

In the sub acute and chronic phase of $\mathrm{SCl}$, inflammatory cells are recruited at the lesion site and forms a scar. Scar mainly composed of a glial scar component and a fibrous scar component. The glial scar in the perilesion area consists predominantly of reactive astrocytes, microglia/macrophages, extracellular matrix (ECM) molecules and chondroitin sulphate proteoglycans (CSPGs) (Yiu and He 2006; Rolls, Shechter et al. 2009). The fibrous scar in the lesion core consists rich basement membrane embedded with collagen type IV (Shearer and Fawcett, 2001). The lesion scar in the SCI remains to be a challenging pathophysiological barrier because of its heterogeneity with its both harmful and beneficial properties on axonal regeneration. Most of the regeneration failure studies found the axon growth arrest and misrouting of axons at the lesion boarder. Studies of axon-astroglial interactions propose that other than glial scar, basement membrane of fibrous scar might be a 
major axon growth inhibition factor of scar tissue (Fawcett et al., 1989; Frisén et al., 1993; Stichel and Müller, 1994; Stichel et al., 1999)

The long-term goal of $\mathrm{SCl}$ research is to institute treatments improving axonal regeneration and establishment of neuronal reinnervation by targeting those identified barriers of axonal regeneration. Figure 4 illustrates possible ways of axonal reinnervation after SCl.

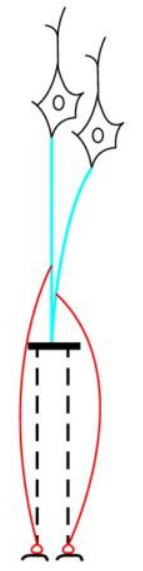

a

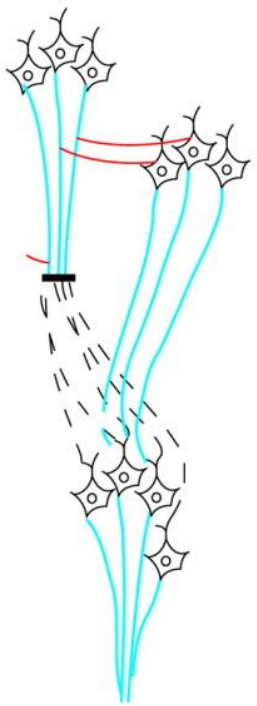

b

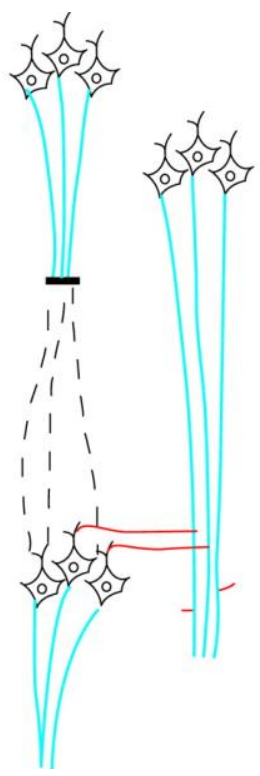

C

Figure 4. Scheme of possible axonal reinnervation ways following SCI. (a) Long-distance axonal regeneration. Following the $\mathrm{SCl}$, the distal segment of the axon undergoes Wallerian degeneration (dotted black lines) and new axons (red) sprout from the proximal axonal segment (blue) to reestablish synaptic contacts with distal axons. (b) Short-distance growth of lesioned axons. Collateral sprouts (red) form synaptic contacts with neighboring axons to by-pass the lesion site. (c) Sprouting of spared distal axons to maintain synaptic connectivity beyond the injury site. Image was adapted from Giger et al., 2010.

\subsection{Current treatment approaches for SCI}

Studies in the last 20 years have made an enormous progress in the understanding of molecular and cellular pathways that are triggered following $\mathrm{SCl}$ and provided insights into key mechanisms of tissue damage and regeneration failure in the CNS. This basic understanding had led to various strategies to repair the $\mathrm{SCl}$ and to increase the functional gain. The main goals for the $\mathrm{SCl}$ research are to improve neuroprotection, increase axonal 
regeneration and foster functional restitution. To achieve these aims different approaches including pharmacological interventions, cell-based therapies, tissue engineering with scaffolds and tissue bridges, rehabilitation with robotics and target-based therapies for scar and myelin inhibitors have been tested in experimental settings.

Cell-based therapies aim to bridge the lesion cyst or cavity with neuroprotective or remyelinating cells to create a favorable environment for axonal regeneration. Various cell types such as olfactory ensheathing glia, Schwann cells and embryonic adult stem cells have been used extensively and are tested in ongoing clinical trials (Geffner et al., 2008; Guest et al., 2013; Knoller et al., 2005; Mackay-Sim et al., 2008). A variety of pharmacological agents such as corticosteroids, GM-1 ganglioside (GM-1), nimodipine, thyrotropin-releasing hormone (TRH), and naloxone showed beneficial effects in preclinical studies of SCl. While their investigated in controlled clinical trials have been failed to demonstrate convincing neurological benefit due to side effects (James W. Rowland et al., 2008; Silva et al., 2014).

One of the most challenging strategies for $\mathrm{SCl}$ treatment is to target major obstacles of axonal regeneration: the myelin inhibitors and the scar tissue in the CNS. Targeting these obstacles could ameliorate the intrinsic neuroprotective and axonal regeneration capacity after the lesion. For example, anti-scarring treatment with the iron chelator BPY-DCA or chondroitinase ABC ameliorated axonal regeneration in vivo (García-Alías et al., 2008; Klapka et al., 2005; Schiwy et al., 2009; Starkey et al., 2012; Tester and Howland, 2008). Targeting myelin inhibitory molecules such as Nogo-A, MAG and downstream effector molecules Rho and ROCK2 improved axonal regeneration in various models of spinal cord injury (Buchli and Schwab, 2005; Dergham et al., 2002; Kastin and Pan, 2005; McKerracher, 2001; Rowland et al., 2008). Ongoing clinical trials are thus focused on Nogo antibodies and BA-210 (Cethrin(®), which is a Rho inhibitor (Fehlings et al., 2011; Filli and Schwab, 2012). 


\subsection{Anti-scarring treatment (BPY-DCA)}

The structure of the fibrous scar component is impermeable for the regrowth of the axons at the lesion site (Fawcett et al., 1989; Frisén et al., 1993; Stichel and Müller, 1994; Stichel et al., 1999). In order to create growth permissive environment at the lesion site, scar suppression treatments are essential. Scar suppression treatments interfering with the synthesis of collagen, a supramolecular backbone of the basement membrane structure of ECM, were found to be an effective strategy to promote axonal growth at the lesion site (Stichel et al., 1999). 2,2'- dipyridine-5, 5'-dicarboxylic acid (BPY-DCA) is an iron chelator that inhibits prolyl-4-hydroxylase, a key enzyme of collagen biosynthesis. Previous studies of anti-scarring treatment with BPY-DCA in combination with 8-bromo-cyclic adenosine monophosphate (8-Br-cAMP) have proven beneficial effects on fibrous scarring and also improved axonal regeneration in a rat spinal cord lesion model (Klapka, Hermanns et al. 2005; Schiwy, Brazda et al. 2009).

\subsection{Gene therapy}

A crucial question in $\mathrm{SCl}$ treatment is the delivery of target cell specific therapeutics to modulate the specific pathways activated after injury. The principle of gene therapy is to use DNA as a drug to express the therapeutic genes in the target cell via a safe vector system. Viral vectors are the efficient tools that could deliver long-lasting cell-specific expression of the therapeutic genes. Adeno-associated viruses (AAV) are small (25 nm in diameter) dependoviruses from the Parvoviridae family. They could replicate only in the presence of a helper virus (either adenovirus or herpes virus). The ability of these viruses to infect dividing and non-dividing cells with stable expression makes them attractive tools for gene therapy (Buller et al., 1981; Daya and Berns, 2008). Recombinant AAV production generates AAV vectors free of helper viral genome while keeping the inverted terminal repeats (ITRs), which are necessary for transgene cassette expression. Because of the lack of viral genome especially the "rep" gene, which is responsible for site-specific integration into chromosomal DNA, it remains episomal and becomes a safe vector for gene therapy studies. Different serotypes (AAV1, 2, 3 etc.) are 
produced based on differences in capsid types, which also affect the expression (Blits et al., 2010; Ruitenberg et al., 2002). The AAV vector system is widely used in CNS research because of its efficient transduction with minimal toxicity and immune response (Blits et al., 2003, 2003; Kaplitt et al., 1994; McCown, 2005, 2005; McCown et al., 1996; Peel and Klein, 2000; Ruitenberg et al., 2004; Taymans et al., 2007; Wu et al., 2006; Zolotukhin et al., 2013). To date AAV gene therapy has been successfully used in animal models of several CNS disorders including spinal cord injury. For example, improved cell survival was observed with AAV vector-mediated expression of the glial cell line-derived neurotrophic factor (GDNF) and Bcl-2 expression in ALS mice (Azzouz et al., 2000; Mandel et al., 1997). Improved neuronal survival and axonal regeneration was also observed in an optic nerve crush model through AAV-mediated over expression of BAG1 or reggie1 (Koch et al., 2013; Planchamp et al., 2008). AAV-mediated BDNF gene therapy ameliorated injured neuronal survival, increased regeneration associated genes such as GAP-43 and Blll-tubulin, and thereby increased axonal sprouting in CST and RST lesion models (Blits et al., 2004; Kobayashi et al., 1997; Kwon et al., 2002a, 2007; Ruitenberg et al., 2004; Vavrek et al., 2006).

Thus, AAV-based gene therapy seems to be a promising strategy for targeted therapies to the red nucleus (RN) in rat models of spinal cord lesion. In this study, the AAV1/2 hybrid serotype that has AAV2 inverted terminal repeats (ITRs) and AAV1 Rep and Cap genes (AAV1/2) was used to induce efficient neuronal expression of the target genes in the CNS (Kiyota et al., 2009; Koch et al., 2013).

\subsubsection{Rho and Rho kinase (ROCK)}

Axon growth and regeneration are regulated by actin cytoskeleton dynamics of the neuronal growth cone and Rho family members are important regulators of actin cytoskeletal dynamics. Rho signal transduction depends on the activation of downstream effector kinases such as ROCK. Thus Rho and ROCK signaling play important roles in the nervous system for axonal 
regeneration and sprouting (Forgione and Fehlings, 2013; Samuel and Hynds, 2010).

ROCK is a serine/threonine protein kinase with a molecular mass of $\sim 160 \mathrm{kDa}$. ROCK has two isoforms, namely ROCK1 and ROCK2, which are encoded by two different genes. Despite similarities in protein sequence, the tissue distribution of ROCK1 and ROCK2 is quite different, representing the functional differences of these isoforms in vivo. ROCK1 is predominantly expressed in non-neuronal tissues such as heart, lung and skeletal muscle. ROCK2 is mainly expressed in the brain tissue and high levels of ROCK2 expression were reported during postnatal development of the mouse brain, especially in the Purkinje cells of the cerebellum, the cerebral cortex and hippocampal neurons (Hashimoto et al., 1999; Komagome et al., 2000; Matsui et al., 1996; Nakagawa et al., 1996).

One of the reported mechanisms of action of ROCK on spine stability and growth cone rearrangement is via phosphorylation of LIM-kinase 1 (LIMK1), which in turn inactivates cofilin, an actin depolymerizing factor (Arber et al., 1998; Ohashi et al., 2000). Recently, upregulation of ROCK2 was reported after spinal cord injury in vivo (Wu et al., 2013). It has been shown previously that inhibition of Ras homolog gene family member A (RhoA) or ROCK can improve axonal outgrowth in vitro and axonal regeneration after CST lesion and optic nerve crush injury in vivo (Dergham et al., 2002; Lehmann et al., 1999; Lingor et al., 2007). Lentiviral-mediated expression of dominantnegative ROCK demonstrated increased axonal sprouting and partial motor recovery in an RST lesion model in vivo (Wu et al., 2009). Most importantly, inhibition of ROCK has also been demonstrated to improve neuronal survival in animal models of Parkinson's disease and amyotrophic lateral sclerosis (Tönges et al., 2012, 2014). Thus modulation of ROCK represents a promising therapeutic target in $\mathrm{SCl}$ research (Tönges et al., 2011; Watzlawick et al., 2013). Figure 5 depicts the scar tissue and mechanism of Rho-ROCK signaling and myelin inhibitory pathways on growth cone arrest after spinal cord lesion. 


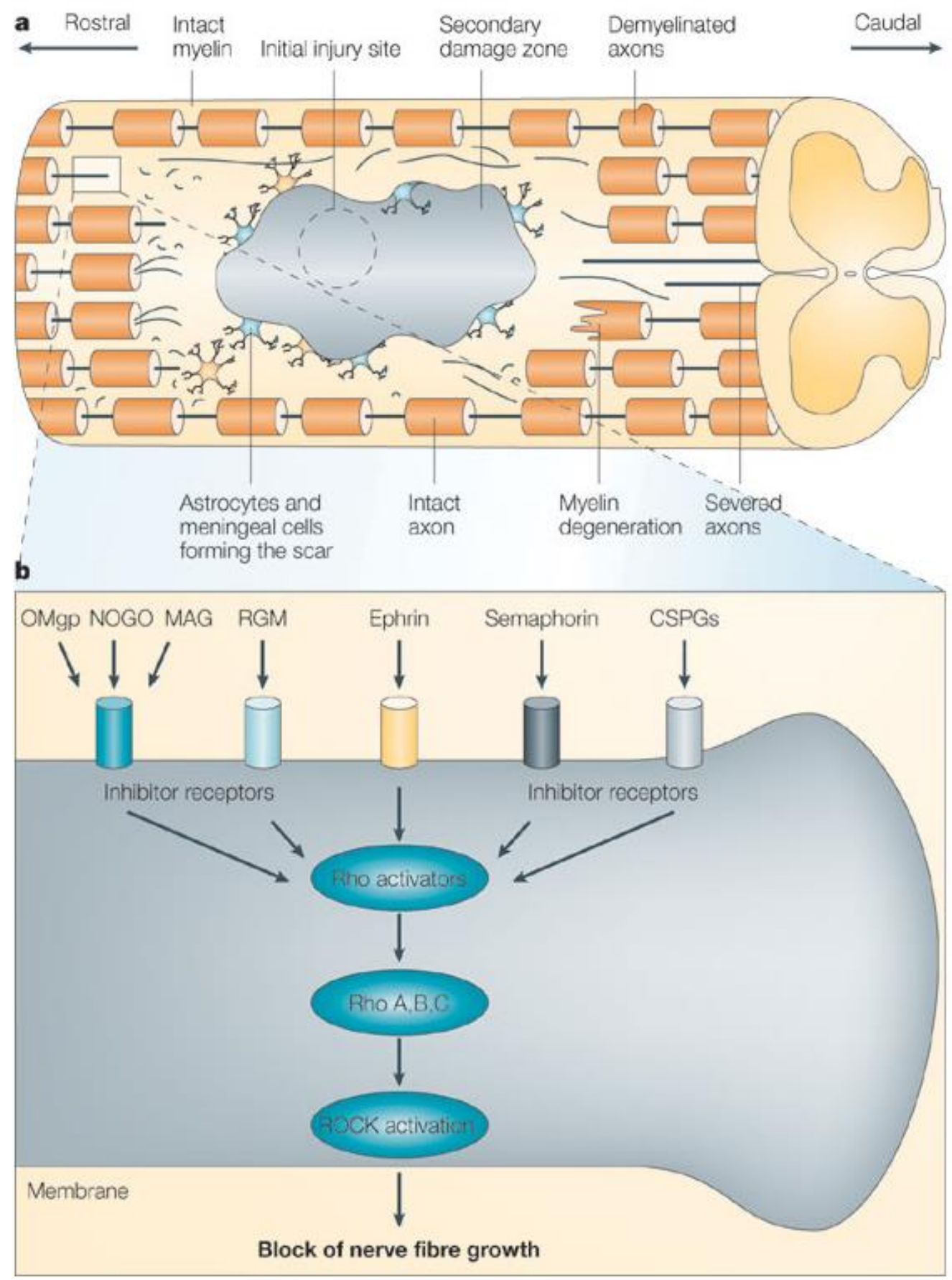

Figure 5. Inhibitory pathways for axonal regeneration following SCl. (a) Schematic view of the SCI displaying scar tissue formation by the activated astrocytes and meningeal cells, which form a growth impermeable environment for axons. (b) Activation of myelin inhibitors OMgp, Nogo, MAG, CSPGs and inhibitory guidance cues, e.g. ephrins and semaphorins, results in subsequent activation of the Rho-ROCK pathway finally leading to the inhibition of nerve fiber growth. Image was adapted from Mueller et al., 2005. 


\subsubsection{BAG1}

Bcl-2-associated athanogene-1 (BAG1) is a multifunctional protein binding to Hsp70/Hsc70, which was previously shown to be neuroprotective, antiapoptotic and to promote neuronal differentiation (Götz et al., 2005; Takayama et al., 1995). Overexpression of BAG1 had neuroprotective effects in rodent stroke models via its anti-apoptotic activity and ameliorated huntingtin toxicity and aggregation in Huntington's disease models in vitro and in vivo (Jana and Nukina, 2005; Sroka et al., 2009). In addition to its neuroprotective effects, BAG1 also induced increased neurite lengths in vitro (Kermer et al., 2002). BAG1 mediates its effects on cell growth and differentiation via activating the rapidly accelerated fibrosarcoma-1 (Raf-1) kinase and its downstream extracellular signal regulated kinase (ERK) (Song et al., 2001). Studies in an optic nerve crush model demonstrated that BAG1 overexpression in retinal ganglion cells resulted in improved cell survival and axonal regeneration via activation of Raf- 1 signalling and reduction of ROCK activity (Planchamp et al., 2008) (Figure 6). 


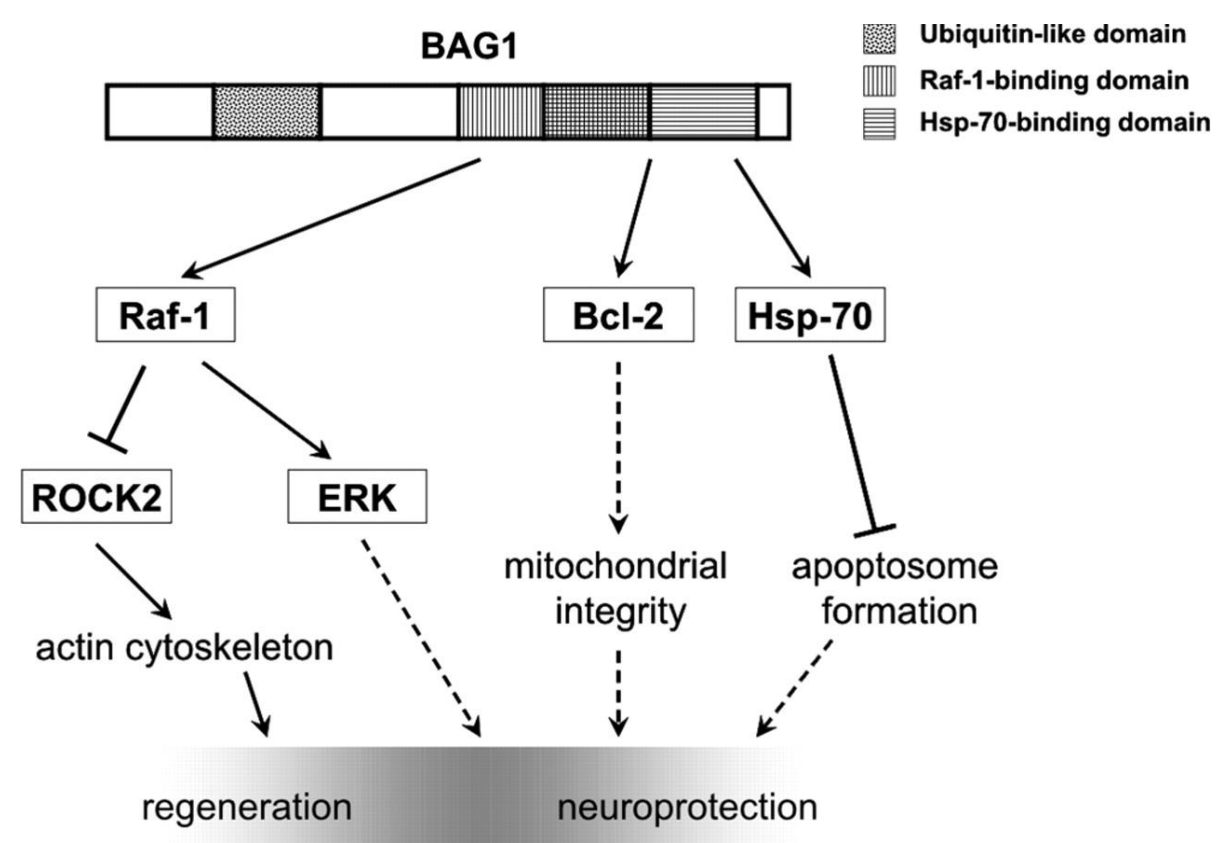

Figure 6. Schematic model of BAG1-mediated neuroprotection and axonal regeneration. BAG1 mediates the activation of the ERK pathway and inhibition of the ROCK activity via Raf-1 activation. Activation of ERK may enhance the intrinsic capability of neuronal outgrowth, while decrease in ROCK activity may regulate actin cytoskeleton rearrangement in the grouth cones. BAG1 activates Bcl-2 and Hsp-70 by its Hsp70-binding domain thus enhancing neuronal survival. Full lines: Direct interaction; Dashed lines: Indirect interaction. Pointed end: stimulation, bar end: inhibition. Image was adapted from Planchamp et al., 2008.

\subsection{3 microRNA-134}

Micro-RNAs (miRNAs) are a family of small non-coding RNAs ( 21-25 nucleotides) that regulate mRNA translation by imperfect base pairing interaction with protein-coding mRNAs. Depending on the degree of sequence complementarity at the $3^{\prime}$-untranslated region of the mature mRNAs they can lead to either a cleavage or a reduction in its translational efficiency of the target mRNA (Zhang et al., 2012). miRNA-134 is a brainspecific miRNA, which is expressed in the rat dendrite spine compartments of hippocampal pyramidal neurons. It was reported to regulate cell proliferation and apoptosis in the lung septa development (Zhang et al., 2012). miRNA134 was also reported to regulate dendrite spine development via BDNF and LIMK1, which is a direct target of miRNA-134 (Numakawa et al., 2011; 
Schratt et al., 2006). LIMK1 is a kinase, which is known to regulate the actin cytoskeleton and could thus be responsible for miRNA-134 effects on axonal regeneration (Numakawa et al., 2011; Schratt et al., 2006). Since LIMK1 is a well-known target of ROCK, miRNA-134 indirectly can regulate the same pathways, which are regulating axonal outgrowth following injury (Ohashi et al., 2000). This renders miRNA-134 an especially interesting target for studying axonal regeneration in the CNS.

\subsubsection{Reggie1}

Reggie proteins (reggie1 and reggie2) are two cell surface proteins, which were first discovered in retinal ganglion cells (RGC) of the goldfish during axonal regeneration (Schulte et al., 1997). In an independent study, they were also identified as insoluble floating lipid raft fraction the membranes, hence called flotillins (Bickel et al., 1997) and are (reggie1/flotillin2 and reggie2/flotillin1) used as lipid raft markers. Both proteins are evolutionary conserved, ubiquitously expressed in various cell types and are well-known for selected cargo transport to specific target cells areas such as cell-cell contacts, T-cell caps, and growth cones (Stuermer, 2010). Recent studies had given some insight on the possible molecular mechanism of reggie in axonal regeneration in mammals. Down-regulation of reggie leads to abnormally bulged, malformed growth cones, reduced axonal regeneration, growth cone elongation in zebra fish and reduced cell differentiation of hippocampal neurons (Munderloh et al., 2009). Further, down-regulation of reggie has also led to an imbalance in activation of Rho GTPases, Rac, Rho, and cdc42, which in turn activated downstream effectors such as Ras, cofilin and focal adhesion kinase (FAK) (Langhorst et al., 2008; Munderloh et al., 2009; Schrock et al., 2009). In support of this data, reggie was also reported to recruit cellular prion protein (PrPc)-mediated $\mathrm{N}$-cadherin at cell-cell contacts, which further strengthens its role in controlling actin cytoskeleton turnover through Rho-GTPases (Ren et al., 2013; Solis et al., 2012; Stuermer, 2010). Recently, expression of reggie1/flotillin2 was reported to be down-regulated following acute $\mathrm{SCl}$, but upregulated in later phases and intrathecal supply of reggie1 antisense-oligonucleotide improved functional 
recovery in a rat model (Santiago et al., 2013). In contrast, our laboratory has shown that AAV-mediated over-expression of reggie1/flotillin2 improved axonal regeneration in an optic nerve crush model of rat (Koch et al., 2013).

\subsection{Aims of the thesis}

The current study was designed to evaluate the potential of AAV-based gene therapy by overexpression of BAG1, reggie1, miRNA-134, or shRNAmediated downregulation of ROCK2 alone and in combination with an antiscarring treatment (AST) in an established CNS lesion model, the dorsal spinal cord hemisection. The target molecules were chosen to combine both neuroprotective and pro-regenerative properties and the AST with the ironchelating agent BPY-DCA was chosen to ameliorate the scar formation further improving axonal regeneration. To this point, several substudies were designed:

1. A pilot study aimed at investigating the effects of a combination treatment with AAV-based over-expression of BAG1 and local application of BDY-DCA on axonal sprouting and motor recovery at 16 weeks after dorsal spinal cord hemisection in rats.

2. A further study aimed to evaluate the method of anterograde tract tracing of the RST with biotinylated dextran amine (BDA) and AAVmediated expression of the fluorophores DsRed (AAV.DsRed) and EGFP (AAV.EGFP) after the dorsal spinal cord hemisection in the rat.

3. Finally the effect of different target gene therapies by AAV-based overexpression of BAG1, reggie1, miRNA-134 and ROCK2-shRNA was investigated. Here, the effect of AAV-mediated gene therapy on RN neuronal survival, axonal sprouting of RST and motor recovery at 12 weeks after unilateral dorsal spinal cord hemisection was studied. In addition to the evaluation of the target gene therapies, we aimed to demonstrate feasibility and limitations of AAV-mediated protein overexpression vs. shRNA- or miRNA-mediated downregulation in this model of spinal cord injury. 


\section{Materials}

\subsection{Instruments}

Table 1. List of the instruments used in this study.

Instrument

ApotomeTM

Autoclave

Dental drill

CCD camera AxioCam

Cryostat

Freezers $\left(-80^{\circ} \mathrm{C}\right)$

Ice machine

Micro4 smart controller

Microwave oven

Nanoliter2000 injector

Peristaltic pump, IP4

$\mathrm{pH}$-meter

Pipettes

Refrigerators $\left(4^{\circ} \mathrm{C}\right.$ and $-20^{\circ}$ C)

Scales

Stereotactic frame

Surgery microscope

Surgical and dissection tools

Surgical suture

Osmotic mini-pumps

Table centrifuges

Ultracentrifuge Sorvall

Video camcorder

Vortex mixer

Water bath

Water purifier, PureLab Plus
Company

Zeiss, Göttingen, Germany

Systec, Greiz, Germany

P10 burr, Meisingen, Germany

Zeiss, Göttingen, Germany

Leica, Nussloch, Germany

Heraeus, Hanau, Germany

Scotman® Frimont, Milan, Italy

World Precision Instruments, Florida, USA

Bosch, Stuttgart, Germany

World Precision Instruments, Florida, USA

Ismatec, Glattbrugg,Switzerland

Sartorius, Göttingen, Germany

Gilson, Villiers le Bel, France

Liebherr, Biberach, Germany

Sartorius, Göttingen, Germany

David Kopf Instruments, California, USA

Zeiss, Göttingen, Germany

Fine Sceince Tools, Heidelberg, Germany

Ethicon, Johnson-Johnson, Livingston, U.K

Model-2004, ALZET ${ }^{\circledR}$, Cupertino, CA

Eppendorf, Hamburg, Germany

DuPont Instruments, Bag Homburg, Germany

Canon LEGRIA-HF M36, Miami, USA

NeoLab, Heidelberg, Germany

GFL, Burgwedel, Germany

ElgaLabwaters, Ransbach-Baumbach, Germany 


\subsection{Chemicals and Consumables}

Table 2. List of chemicals and consumables used in this study.

Product

Bovine serum albumine (BSA)

Brauno|ß

2,2'-bipyridine-5, 5'dicarboxylic acid (BPY-DCA)

Cryomatrix

D (+)-glucose

D (+)-sucrose

Ethanol absolute

Formaldehyde

Glycerine

Glycerol

Glycine

Hydrogen peroxide $\left(\mathrm{H}_{2} \mathrm{O}_{2}\right)$

Magnesium chloride $\left(\mathrm{MgCl}_{2}\right)$

Mowiol

Normal goat serum

N-propyl-gallate (NGS)

Paraformaldehyde (PFA)

PBS (1x Dulbecco's, powder)

Sodium acetate

Sodium azide $\left(\mathrm{NaN}_{3}\right)$

Sodium bicarbonate $\left(\mathrm{Na}_{2} \mathrm{CO}_{3}\right)$

Sodium chloride $(\mathrm{NaCl})$

Sodium hydroxide $(\mathrm{NaOH})$

TRIS-base

Triton X-100

Tween20
Reference

Sigma, Taufkirchen, Germany

(B.Braun Melsungen AG, Berlin, Germany)

Sigma, Taufkirchen, Germany

Cryomatrix (ThermoScientific, Schwerte, Germany),

Sigma, Taufkirchen, Germany

AppliChem, Darmstadt, Germany

AppliChem, Darmstadt, Germany

Merck, Darmstadt, Germany

Sigma, Taufkirchen, Germany

Roth, Karlsruhe, Germany

AppliChem, Darmstadt, Germany

Merck, Darmstadt, Germany

AppliChem, Darmstadt, Germany

CalbioChem, La Jolla, CA, USA

PAA, Coelbe, Germany

Sigma, Taufkirchen, Germany

AppliChem, Darmstadt, Germany

AppliChem, Darmstadt, Germany

Fluka, Taufkirchen, Germany

Sigma, Taufkirchen, Germany

Sigma, Taufkirchen, Germany

AppliChem, Darmstadt, Germany

AppliChem, Darmstadt, Germany

AppliChem, Darmstadt, Germany

Sigma, Taufkirchen, Germany

Fluka, Taufkirchen, Germany 
All general laboratory chemicals and consumables were purchased from AppliChem, (Darmstadt, Germany) and Sarstedt (Nümbrecht, Germany) unless otherwise specified.

\subsection{Solutions and Buffers}

\subsubsection{Mowiol}

$5 \mathrm{~g}$ of mowiol were dissolved in $20 \mathrm{ml}$ sterile PBS under constant stirring for 4 $\mathrm{h}$ at $4^{\circ} \mathrm{C}$, to which $10 \mathrm{ml}$ of glycerine were added and dissolved on a shaker for $16 \mathrm{~h}$ at $4^{\circ} \mathrm{C}$. The mixture was then centrifuged for $15 \mathrm{~min}$ at $4000 \mathrm{rpm}$ $(1699 \mathrm{~g})$ and the supernatant was removed carefully. The $\mathrm{pH}$ was adjusted to 8.0 and finally $0.3 \mathrm{ml}$ of sodium azide $(2 \% \mathrm{w} / \mathrm{v})$ and a tip of $\mathrm{N}$-propyl-gallate were added to $10 \mathrm{ml}$ of the solution, which was centrifuged for $20 \mathrm{~min}$ at 4000 rpm (1699 g).

\subsubsection{Paraformaldehyde (PFA) 4\%}

$40 \mathrm{~g}$ of PFA were dissolved in $700 \mathrm{ml}$ of filtered PBS under constant heating $\left(55^{\circ} \mathrm{C}\right)$. A small pellet of $\mathrm{NaOH}$ was added to the PBS to enhance the dissolution of PFA. pH was carefully adjusted to 7.4 and the solution was made up to $1000 \mathrm{ml}$ with PBS was stored at $4^{\circ} \mathrm{C}$.

\subsubsection{1x PBS}

$9.55 \mathrm{~g}$ of PBS powder were dissolved in $1000 \mathrm{ml}$ of distilled $\mathrm{H}_{2} \mathrm{O}$, sterilized and filtered.

\subsection{4 $10 \%, 20 \%, 30 \%$ of sucrose (sucrose for cryoprotection)}

$10 \mathrm{~g}, 20 \mathrm{~g}$ or $30 \mathrm{~g}$ of sucrose were dissolved in $100 \mathrm{ml}$ of PBS to prepare 10 $\%, 20 \%$ and $30 \%$ of sucrose, respectively. 


\subsubsection{0\% Normal Goat Serum (NGS)}

$1000 \mu \mathrm{l}$ of NGS were dissolved in $10 \mathrm{ml} 1 \times$ PBS.

\subsubsection{Citrate buffer (antigen retrieval solution)}

$0.21 \mathrm{~g}$ of citric acid monohydrate $(0.1 \mathrm{M})$ were dissolved in $100 \mathrm{ml}$ of distilled $\mathrm{H}_{2} \mathrm{O}$. The $\mathrm{pH}$ was adjusted to 6 with $1 \mathrm{M} \mathrm{NaOH}$.

\subsubsection{Blocking solution for immunohistochemistry}

$100 \mu \mathrm{l}$ of $10 \%(\mathrm{w} / \mathrm{v})$ normal goat serum, $0.01 \mathrm{~g}$ of bovine album serum, $0.3 \%$ $(\mathrm{w} / \mathrm{v})$ Triton $\mathrm{X}-100$ were added to $1 \mathrm{ml}$ of $1 \times$ PBS.

\subsubsection{TRIS buffer, $20 \mathrm{mM}, \mathrm{pH} 8.1$}

$2.1 \mathrm{~g}$ of TRIS base $\left(\mathrm{C}_{4} \mathrm{H}_{11} \mathrm{NO}_{3}\right)$ was dissolved in $1000 \mathrm{ml}$ of distilled $\mathrm{H}_{2} \mathrm{O}$. The $\mathrm{pH}$ was adjusted to 7.9 with $\mathrm{HCl}$.

2.3.9 BPY-DCA (2,2'-bipyridine-5, 5'-dicarboxylic acid), 40 mM, pH 7.4 Solution 1: $20.1 \mathrm{mg}$ of BPY-DCA powder were dissolved in $2.06 \mathrm{ml}$ TRIS buffer ( $\mathrm{pH} 5.5)$.

Solution 2: $20 \mu \mathrm{l} 5 \mathrm{M} \mathrm{NaOH}(\mathrm{pH}$ 6.1) were added to $1.9 \mathrm{ml}$ of solution 1 . Solution 3: $5 \mu \mathrm{l} 5 \mathrm{M} \mathrm{NaOH}(\mathrm{pH} 8.4$ ) were added to $1.7 \mathrm{ml}$ of solution 2 and finally the $\mathrm{pH}$ was adjusted to 7.4 with $\mathrm{HCl}$. 


\subsection{Primary Antibodies}

Table 3. List of primary antibodies used in this study

\begin{tabular}{ll} 
Antibody & Company \\
\hline Mouse anti-NeuN (1:200) & Millipore (Billerica, USA) \\
Goat anti-ROCK2 (1:50) & Santa Cruz Biotechnology (Texas, USA) \\
Rabbit anti-BAG1 (1:250) & Santa Cruz Biotechnology (Texas, USA) \\
Rabbit anti-GAP43 (1:500) & Abcam (Cambridge, UK) \\
Rabbit anti-synaptophysin (1:1000) & Sigma Aldrich (St. Louis, USA) \\
Rabbit anti-GFAP (1:200) & Dako (Hamburg, Germany) \\
Chicken anti-GFP (1:250) & Abcam (Cambridge, UK)
\end{tabular}

\subsection{Secondary Antibodies}

Table 4. List of secondary antibodies used in this study.

\begin{tabular}{|c|c|}
\hline til & y \\
\hline $\begin{array}{l}\text { Alexa Fluor }{ }^{\circledR} 488 \text { conjugated goat } \\
\text { anti-chicken antibody }\end{array}$ & $\begin{array}{l}\text { Invitrogen-Molecular Probes } \\
\text { (Carlsbad, USA) }\end{array}$ \\
\hline $\begin{array}{l}\text { Alexa Fluor }{ }^{\circledR} 647 \text { conjugated donkey } \\
\text { anti-goat antibody }\end{array}$ & $\begin{array}{l}\text { Invitrogen-Molecular Probes } \\
\text { (Carlsbad, USA) }\end{array}$ \\
\hline Cy5 conjugated goat anti-mouse antibody & Dianova (Hamburg, Germany) \\
\hline Су3 conjugated goat anti-mouse antibody & Dianova (Hamburg, Germar \\
\hline Сy3 conjugated goat anti-rabbit & Dianova $(\mathrm{H}$ \\
\hline Cy2 conjugated goat anti-rabbit antibody & Dianova (Hamburg, Germany) \\
\hline y2 conjugated goat anti-mouse antibody & Dianova (Hamburg, Germany) \\
\hline
\end{tabular}


2.6 Anterograde tracing dyes

Table 5. List of anterograde tracers used in this study.

Tracer Dye Company

Biotinylated dextran amine (BDA) Invitrogen-Molecular Probes

MW 10, 1000 (Carlsbad, USA)

Alexa Fluor ${ }^{\circledR} 647$ conjugated cholera Invitrogen-Molecular Probes toxin subunit $B(C T B)$ (Carlsbad,USA) 


\section{Methods}

\subsection{Animals}

Adult female Wistar rats weighing 230-250g were used for all experiments (Charles River, Germany). Animals were maintained at 12/12 h light and dark cycle with ad libitum food and water. All animals were sacrificed by $\mathrm{CO}_{2}$ insufflations followed by transcardial perfusions. All animal experiments were performed following the regulations and legislation of the local animal research council of the Lower Saxony State (Braunschweig), Germany.

\subsection{Cloning of viral vectors}

The AAV1/2 vector expressing BAG1 and the fluorophore EGFP under control of a human synapsin promoter (AAV.BAG1) was kindly provided by Sebastian Kügler (Kügler et al., 2001; Planchamp et al., 2008). All other AAV were kindly provided by Uwe Michel and Jan Koch. Viral vector maps are shown in Annexes, supplementary images.

The control vectors AAV1/2-EGFP (AAV.EGFP) (Gen-Bank ID: HQ416702) and AAV1/2-DsRed (AAV.DsRed) (Gen-Bank ID: AY640633) expressing EGFP or DsRed driven by a human synapsin promoter and the nonsense protein CytBAS under control of a $\mathrm{H} 1$ promoter were used. The cloning of these vectors has been described earlier by Koch et al., 2011.

The AAV1/2-reggie1 (AAV.reggie1) expressing a fusion-protein of reggie-1 and EGFP under control of a human synapsin promoter was used and been described earlier by Koch et al., 2011.

The AAV1/2-shRNA-ROCK2 (AAV.sh.ROCK2), AAV1/2-shRNA-firefly (AAV.sh.firefly) and AAV1/2-miRNA-134 (AAV.miRNA-134)) were expressing the respective shRNA or miRNA-134 under control of a $\mathrm{H} 1$-promoter and the fluorophore EGFP driven by a human synapsin promoter were used. They were cloned according to a protocol published elsewhere (Michel et al., 2005). In brief, $200 \mathrm{pmol}$ of the forward and reverse primers containing the previously tested and confirmed shRNA sequence were dissolved in $25 \mu \mathrm{l}$ 
water and $25 \mu$ l of $2 x$ annealing buffer (20 mM TRIS, pH 7.8, $100 \mathrm{mM} \mathrm{NaCl}$, and $0.2 \mathrm{mM}$ EDTA). The solution was boiled for $5 \mathrm{~min}$ and then cooled to room temperature. Annealed oligos $(200 \mathrm{fmol})$ were ligated to $22.5 \mathrm{fmol}$ of the Bgl2/Hind3-cut backbone fragment of pSUPER-hSyn-EGFP-CytB-AS (GenBank ID: AY640629; Michel et al., 2005).

The sequences of the shRNA primers were as follows (in bold: sequence of the respective siRNA-sense and -antisense strand, in italics: sequence of the hairpin turn):

For AAV.sh.ROCK2:

ROCK2-shRNA forward primer:

GATCCCC-TGCAAAGTTTATTATGATATA-CTTCCTGTCA-

TATATCATAATAAACTTTGCA-TTTTTGGAAA;

ROCK2-shRNA reverse primer:

AGCTTTTCCAAAAA-TGCAAAGTTTATTATGATATA-

TGACAGGAAG- TATATCATAATAAACTTTGCA-GGG.

For AAV.sh.firefly (see Malik et al., 2006):

Luciferase-2 forward primer:

GATCCCCGTGCGCTGCTGGTGCCAACCCCTTCCTGTCAGGGTTG GCACCAGCAGCGCACTTTTTGGAAA.

Luciferase-2 reverse primer

AGCTTTTCCAAAAAGTGCGCTGCTGGTGCCAACCCTGACAGGA AGGGGTTGGCACCAGCAGCGCACGGG;

For AAV.miRNA-134:

miRNA-134 forward primer

CAGGGTGTGTGACTGGTTGACCAGAGGGGCGTGCACTTTGTTC ACCCTGTGGGCCACCTAGTCACCAACCCTC;

miRNA-134 reverse primer

GAGGGTTGGTGACTAGGTGGCCCACAGGGTGAACAAAGTGCAC GCCССTCTGGTCAACCAGTCACACACCCTG. 
This step resulted in the vectors pSUPER-hSyn-EGFP-H1-ROCK2-shRNA and pSUPER-hSyn-EGFP-H1-luc2 that were then digested with BstB1/Hind3. The resulting inserts containing the $\mathrm{H} 1$-promoter and the ROCK2- or fireflyshRNA were cloned into a BstB1/Hind3-cut pAAV-9(5)hSyn-EGFP-CytbASohneNot (Gen- Bank ID: HQ416702) resulting in the vector pAAV-9(5)hSynEGFP-H1-ROCK2-shRNA or pAAV-9(5)hSyn-EGFP-H1-firefly-shRNA or pAAV-9(5)hSyn-EGFP-miRNA-134 which was then used for AAV production.

Production of AAV (serotype 1/2) was performed as reported before (Zolotukhin S et al., 1999). Briefly, 293-HEK cells were transfected with calcium phosphate, HEPES buffered saline and a plasmid mix of pAAV-RC, pH21, pHELPER and the respective pAAV expression vector in a 0.5:0.5:1:1 molar ratio. The $\mathrm{pH} 21$ vector, expressing AAV serotype 1 capsids, was a gift from Helen Fitzsimons (Neurologix, Inc. OSU Comprehensive Cancer Center, Columbus, $\mathrm{OH}$ ) and Matthew During (Molecular Virology, Immunology, and Medical Genetics, Columbus, $\mathrm{OH}$ ). $48 \mathrm{~h}$ after transfection, cells were harvested and AAV were purified by dialysis and virus gradient centrifugation in iodixanol. Fast protein liquid chromatography (FPLC) was performed to obtain high titer viral stocks. The virus stocks were then tested on primary cortical neurons for transduction efficacy and toxicity and viral titers were determined using qPCR.

\subsection{Experimental set-up and animal groups}

3.3.1 Pilot project: Effects of the combination of BAG1 overexpression and anti-scarring treatment with BPY-DCA in a rat model of dorsal spinal cord hemisection.

A list of treatment groups is given in Table 6 and the sketch of the experimental plan is shown in Figure 7. 
Table 6. List of treatment groups for combination of BAG1 with antiscarring treatment

\begin{tabular}{|c|c|c|c|c|}
\hline Group & $\begin{array}{l}\text { AAV injections in the } \\
\text { RN }\end{array}$ & $\begin{array}{l}\text { Short name } \\
\text { used in this } \\
\text { thesis }\end{array}$ & 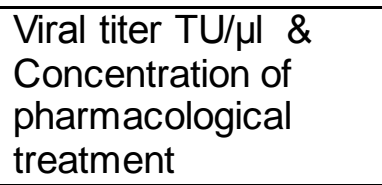 & $\begin{array}{l}\text { Number } \\
\text { of } \\
\text { animals }\end{array}$ \\
\hline$A$ & AAV1/2-BAG1-EGFP & AAV.BAG1 & $1 \times 10^{8}$ & 6 \\
\hline$B$ & $\begin{array}{l}\text { AAV1/2-BAG1-EGFP } \\
+ \text { BPY-DCA }\end{array}$ & $\begin{array}{l}\text { AAV.BAG1 + } \\
\text { AST * }\end{array}$ & $\begin{array}{l}1 \times 10^{8}+40 \mathrm{mM}(\mathrm{pH} \\
7.4)\end{array}$ & 6 \\
\hline $\mathrm{C}$ & $\begin{array}{l}\text { AAV1/2-BAG1-EGFP } \\
+ \text { TRIS buffer }\end{array}$ & $\begin{array}{l}\text { AAV.BAG1 + } \\
\text { ASB * }\end{array}$ & $\begin{array}{l}1 \times 10^{8}+20 \mathrm{mM}(\mathrm{pH} \\
8.1)\end{array}$ & 6 \\
\hline D & $\begin{array}{l}\text { AAV1/2-EGFP (as a } \\
\text { control of A) }\end{array}$ & AAV.EGFP & $3.4 \times 10^{7}$ & 6 \\
\hline$E$ & $\begin{array}{l}\text { AAV1/2-EGFP + } \\
\text { BPY-DCA (as a } \\
\text { control of B) }\end{array}$ & $\begin{array}{l}\text { AAV.EGFP + } \\
\text { AST* }^{*}\end{array}$ & $\begin{array}{l}3.4 \times 10^{\prime}+40 \mathrm{mM}(\mathrm{pH} \\
7.4)\end{array}$ & 6 \\
\hline$F$ & $\begin{array}{l}\text { AAV1/2-EGFP + } \\
\text { TRIS buffer (as a } \\
\text { control of C) }\end{array}$ & $\begin{array}{l}\text { AAV.EGFP + } \\
\text { ASB }^{*}\end{array}$ & $\begin{array}{l}3.4 \times 10^{\prime}+20 \mathrm{mM}(\mathrm{pH} \\
8.1)\end{array}$ & 6 \\
\hline
\end{tabular}

${ }^{*} \mathrm{AST}=$ anti-scarring treatment (BPY-DCA)

${ }^{*} A S B=a n t i-s c a r r i n g ~ b u f f e r$ (TRIS buffer)

All animals were also injected in the cortex with AAV1/2.DsRed $\left(1.4 \times 10^{8}\right.$ TU) in addition to the AAV.BAG1 or AAV.EGFP injections into the RN. The number of animals that were used for data evaluation in each group (after elimination because of foot lesion and insufficient viral transduction at $R N$ ) were AAV.BAG1=3; AAV.EGFP=2; AAV.BAG1 + AST=2; AAV.EGFP + AST $=1 ; A A V \cdot B A G 1+A S B=2 ; A A V \cdot E G F P+A S B=1$. (AST=anti-scarring treatment (BPY-DCA); ASB=anti-scarring buffer (TRIS buffer)).

Elimination criteria of the animals from the study were detailed in the results section 4.1.1. 
a

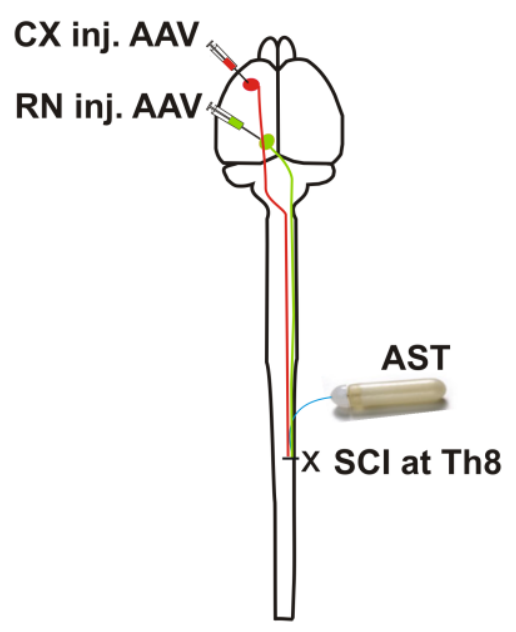

b

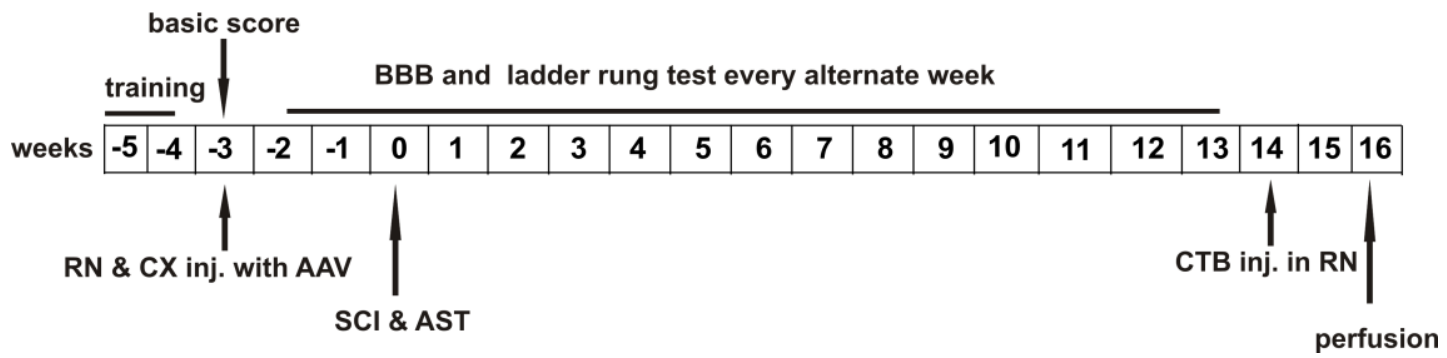

Figure 7. Experimental set-up for the combination treatment with AAV.BAG1 and AST in a rat model of spinal cord dorsal hemisection. (a) Scheme of the experimental set-up showing stereotactic AAV injections in the $R N$ and cortex $(C X)$, dorsal hemisection and osmotic mini-pump implantation (for AST) at thoracic level 8 (Th8). b) Time course of the experiments. $\mathrm{RN}=$ red nucleus; $\mathrm{CX}=\mathrm{cortex} ; \mathrm{AAV}=$ adeno associated virus; $\mathrm{SCl}=$ spinal cord injury, AST=anti-scarring treatment; CTB=cholera toxin B.

3.3.2 Anterograde tracing of RST before and after dorsal spinal cord hemisection

List of treatment groups is given in Table $7 \& 8$. The model sketch of the experiment and the time plan of the experiment are shown in Figures 8 \& 9 
Table 7. List of treatment groups for tracing of RST before and after dorsal spinal cord hemisection.

\begin{tabular}{|c|c|c|}
\hline 1st RN injection & 2nd $\mathrm{RN}$ injection & $\begin{array}{l}\text { Number of } \\
\text { animals }\end{array}$ \\
\hline $\begin{array}{l}\text { AAV1/2-EGFP }\left(3.4 \times 10^{\prime}\right. \\
T U / \mu l)\end{array}$ & $\mathrm{BDA}$ & 3 \\
\hline 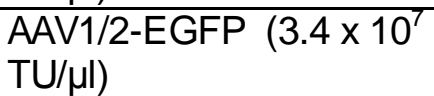 & AAV1/2-DsRed $\left(1.4 \times 10^{8}\right)$ & 2 \\
\hline
\end{tabular}

a

b
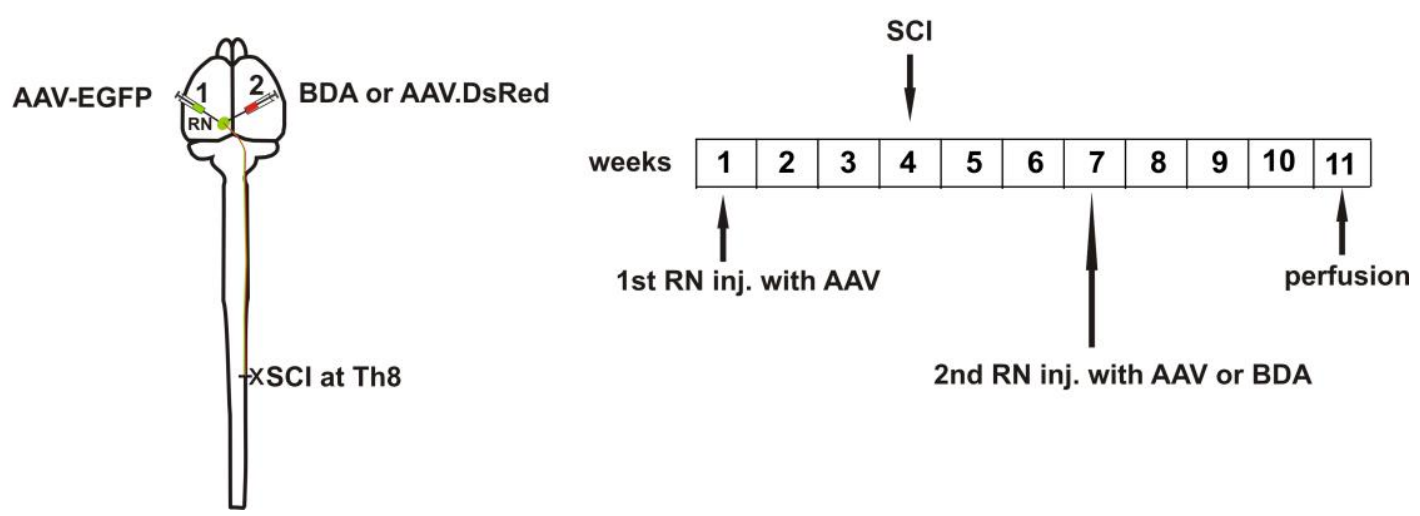

2nd RN inj. with AAV or BDA

Figure 8. Experimental set-up of anterograde tracing of RST before and after dorsal spinal cord hemisection. (a) Scheme of the experimental approach showing stereotactic AAV injections in the $R N$ and dorsal hemisection at thoracic level 8 (Th8). (b) Time course of the experiments. $\mathrm{RN}=$ red nucleus; $\mathrm{AAV}=$ adeno associated virus; $\mathrm{SCl}=$ spinal cord injury; $\mathrm{BDA}=$ biotinylated dextran amine.
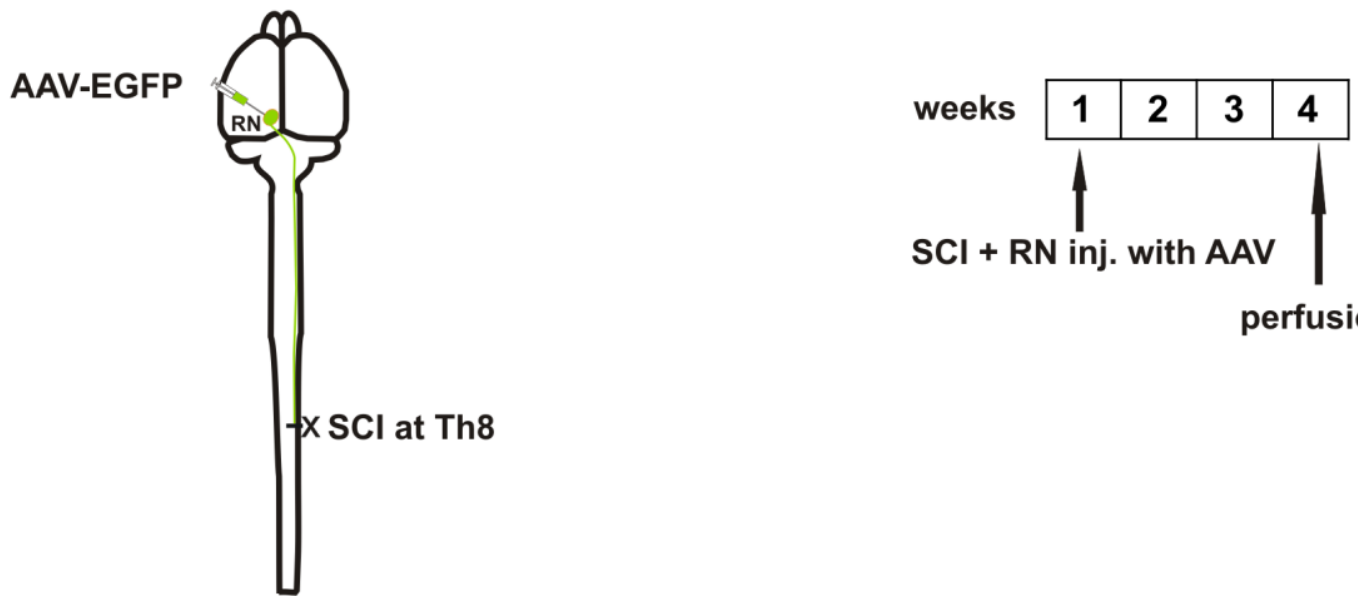

perfusion 
Figure 9. Experimental set-up of anterograde tracing of RST immediately after dorsal spinal cord hemisection. (a) Scheme of the experimental approach showing stereotactic AAV injections in the RN and dorsal hemisection at thoracic level 8 (Th8) (b) Time course of the experiments. $\mathrm{RN}=$ red nucleus; $\mathrm{AAV}=$ adeno associated virus; $\mathrm{SCl}=$ spinal cord injury.

Table 8. List of treatment groups for tracing of the RST immediately after dorsal spinal cord hemisection

\begin{tabular}{|l|l|}
\hline $\begin{array}{l}\text { Anterograde tracing with RN injection immediately after dorsal } \\
\text { spinal cord hemisection }\end{array}$ & $\begin{array}{l}\text { Number of } \\
\text { animals }\end{array}$ \\
\hline AAV1/2-EGFP $\left(3.4 \times 10^{7} \mathrm{TU} / \mathrm{\mu l}\right)$ & 3 \\
\hline
\end{tabular}

3.3.3 Effects of AAV.BAG1, AAV.reggie1, AAV.miRNA-134 and $A A V . s h . R O C K 2$ on RST in a rat model of dorsal spinal cord hemisection List of treatment groups were listed in Table 9. The model sketch of the experiment and the time plan of the experiment are shown in Figure 10.

Table.9 List of treatment groups for gene therapy with AAV.BAG1, AAV.reggie1, AAV.miRNA-134 and AAV.sh.ROCK2 experiment.

\begin{tabular}{|c|l|l|l|c|}
\hline Group & Virus & $\begin{array}{l}\text { Short name used in this } \\
\text { thesis }\end{array}$ & $\begin{array}{l}\text { Viral titer } \\
\text { TU/ } \mu l\end{array}$ & $\begin{array}{l}\text { Number } \\
\text { of } \\
\text { animals }\end{array}$ \\
\hline A & $\begin{array}{l}\text { AAV1/2-EGFP- } \\
\text { miRNA-134 }\end{array}$ & AAV.miRNA-134 & $7.2 \times 10^{8}$ & 10 \\
\hline B & $\begin{array}{l}\text { AAV1/2-EGFP- } \\
\text { BAG1 }\end{array}$ & AAV.BAG1 & $1 \times 10^{8}$ & 15 \\
\hline C & $\begin{array}{l}\text { AAV1/2-EGFP- } \\
\text { ROCK2- } \\
\text { shRNA }\end{array}$ & AAV.sh.ROCK2 & $3.5 \times 10^{8}$ & 15 \\
\hline D & $\begin{array}{l}\text { AAV1/2-EGFP- } \\
\text { reggie1 }\end{array}$ & AAV.reggie1 & $4.2 \times 10^{8}$ & 9 \\
\hline E & $\begin{array}{l}\text { AAV1/2-EGFP- } \\
\text { firefly-shRNA } \\
\text { (as a control of } \\
\text { A\&C) }\end{array}$ & AAV.sh.firefly & $9.4 \times 10^{8}$ & 11 \\
\hline F & $\begin{array}{l}\text { AAV1/2-EGFP } \\
\text { (as a control of } \\
\text { B \& D) }\end{array}$ & AAV.EGFP & $3.4 \times 10^{\prime}$ & 10 \\
\hline
\end{tabular}


a
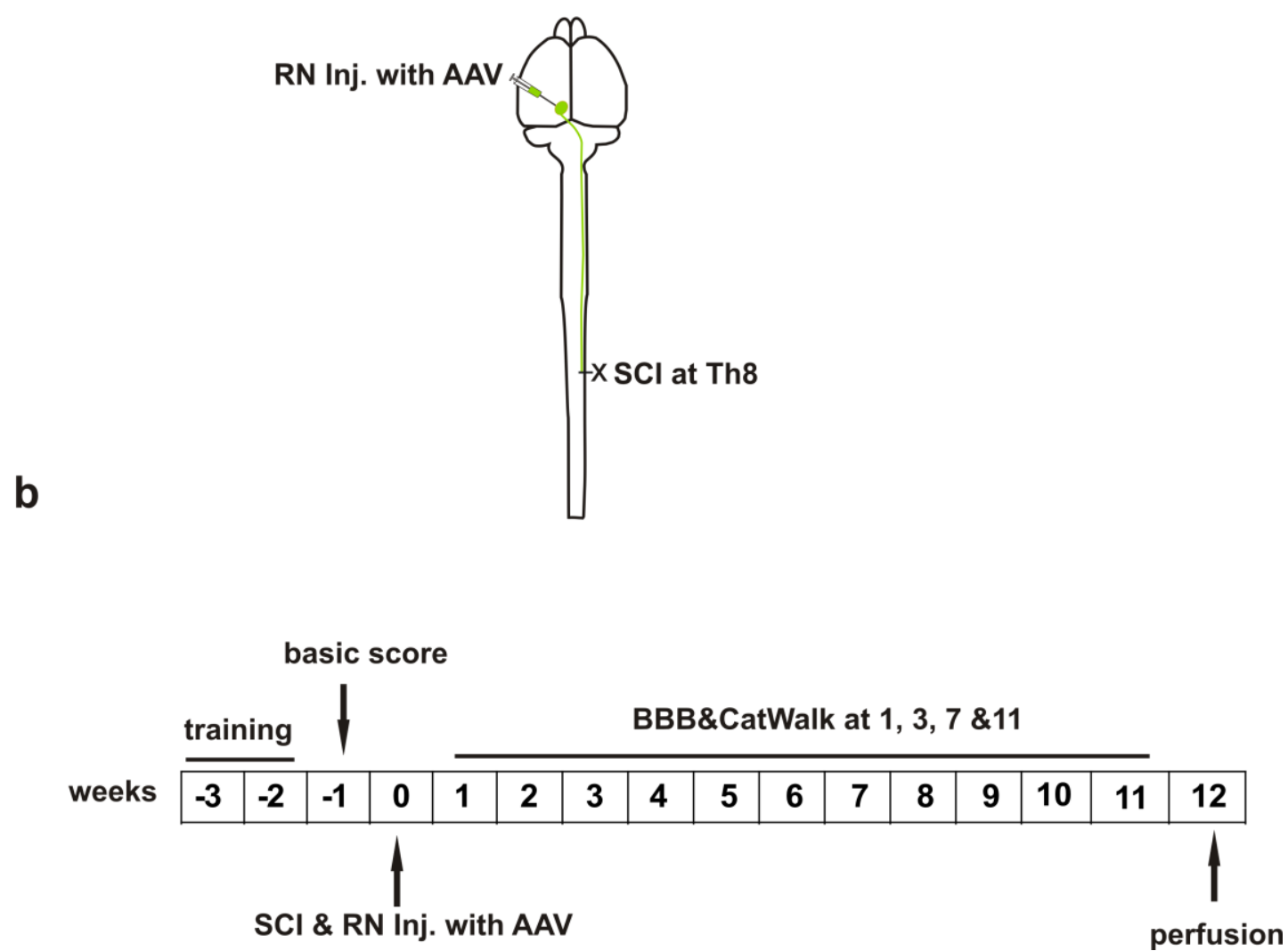

Figure 10. Experimental set-up for anterograde tracing of RST immediately after dorsal spinal cord transection. (a) Scheme of the experimental approach showing stereotactic AAV injections in the $R N$ and dorsal hemisection at thoracic level 8 (Th8). (b) Time course of the experiments. $\mathrm{RN}=$ red nucleus; $\mathrm{AAV}=$ adeno associated virus; $\mathrm{SCl}=$ spinal cord injury.

The number of rats that were used for quantification of the data in this experiment (after elimination of rats due to foot lesion and partial lesion) were AAV.EGFP, $n=4 ;$ AAV.BAG1, $n=8$, AAV.sh.firefly, $n=7$, AAV.sh.ROCK2, $n=8$, AAV.reggei1, $n=7$, AAV.miRNA-134, $n=5$.

\subsection{Animal surgeries}

\subsubsection{Stereotactic injection of AAV into the brain}

Successful gene therapy studies rely on the efficient transduction of the target tissue. The viral injections for the $\mathrm{RN}$ and $\mathrm{CX}$ projections were done according to a previously published protocol (Ruitenberg et al., 2002; 
Wiessner et al., 2003). Rats were deeply anesthetized using 2-3\% Isoflurane anaesthesia in $\mathrm{O}_{2}$ and $\mathrm{NO}_{2}(1: 2)$ and were placed in a stereotaxic frame with horizontal skull position fixed by ear and tooth bars. To expose the skull, an incision in the rostro-caudal direction through the skin was made. The defined stereotactic coordinates for injections were measured with the intersection points of lambdoid and sagittal suture lines (Figure 11), according to the rat brain atlas (Paxinos and Watson, 1998). The heights of lambda and bregma were adjusted to obtain a horizontal skull position. The bregma coordinates for the $\mathrm{RN}$ injections were antereoposterior (AP): -6.0 $\mathrm{mm}$; mediolateral $(\mathrm{ML}):+0.7 \mathrm{~mm}$; and dorsoventral (DV): $-6.8 \mathrm{~mm}$ from the dura mater. The bregma coordinates for the cortex injections were: AP: -2.0 $\mathrm{mm}$; $\mathrm{ML}:+2.4 \mathrm{~mm}$; and $\mathrm{DV}:-1.2 \mathrm{~mm}$ from the dura mater. The distance between the bregma and lambda was measured for all the animals before measuring the coordinates for the injections. The general bregma and lambda distance for the adult Wistar rats was $9 \mathrm{~mm}$ (Paxinos et al., 1985). Based on this distance the coordinates for the RN injections were; (AP): -6.0 $\mathrm{mm}$; mediolateral $(\mathrm{ML}):+0.7 \mathrm{~mm}$; and dorsoventral (DV): $-6.8 \mathrm{~mm}$. The bregma coordinates for the cortex injections were: AP: $-2.0 \mathrm{~mm}$; ML: +2.4 $\mathrm{mm}$; and DV: $-1.2 \mathrm{~mm}$. For any variations in the bregma and lambda distance (generally less than $9 \mathrm{~mm}$ in our case), the coordinates for the $\mathrm{RN}$ and $\mathrm{CX}$ injections were adjusted in correlation to the bregma and lambda length to avoid possible anatomical variations among animals (Paxinos et al., 1985). For e.g., if the bregma and lambda distance was measured as $8.5 \mathrm{~mm}$, the coordinates for the RN injections would be AP: $-5.66 \mathrm{~mm}, \mathrm{ML}:+0.66 \mathrm{~mm}$ and DV: $-6.42 \mathrm{~mm}$. After measuring the coordinates, the target location was marked and a hole was made using a dental drill at the marked reference points. The virus was injected using a heat pulled glass capillary attached to nanolitre2000. The controlled and gradual infusion of AAV into the brain was performed using a micro injector pump system at a speed of $0.3 \mu \mathrm{l} / \mathrm{min}$. The applied volume of virus (Table 6, 7, 8 and 9) or anterograde tracing dye BDA or CTB was $2 \mu$ l. Finally, the skull was closed by suturing the skin using a 3/0 non-absorbable surgical suture. After the experiment, the animals were kept on a warming pad until awake and were then brought back to the home cage. The animals were given postoperative care with an analgesic agent 
(Buprenorphine; 0,05 $\mathrm{mg} / \mathrm{kg}$ body weight) for 3 days.

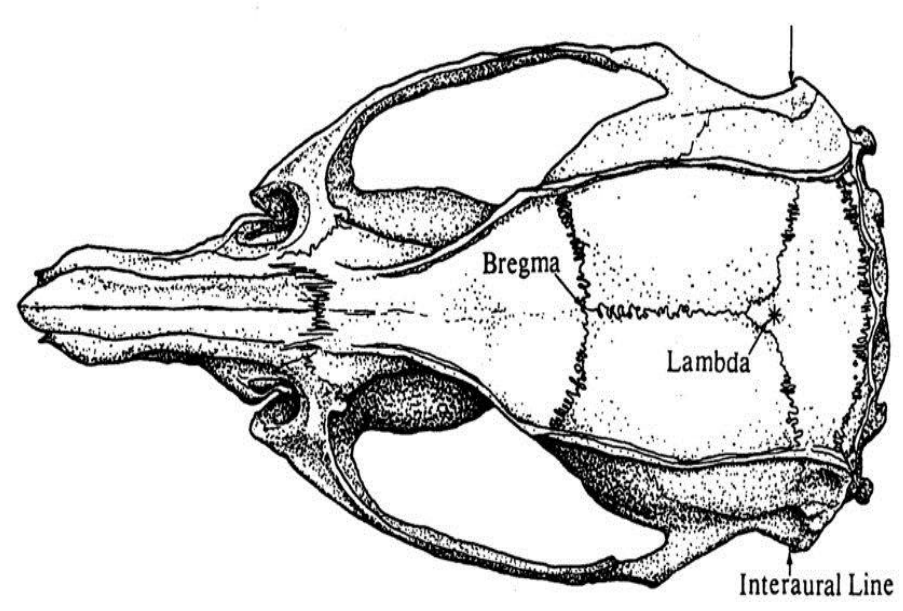

Figure 11. Rat skull suture lines locating the bregma and lambda intersect points for calculating coordinates for stereotactic brain injections. Figure from Paxinos and Watson, 1998.

\subsubsection{Right dorsal hemisection of the spinal cord}

The spinal cord lesion was performed as reported by (Ruitenberg et al., 2004). In brief, under 2-3\% Isoflurane anaesthesia in $\mathrm{O}_{2}$ and $\mathrm{NO}_{2}$ (1:2), the back of the animal was shaved and disinfected and a longitudinal injection was made using a scalpel. The fat layer on the back muscles was gently separated from the muscles and fixed aside with the help of a hook. The back muscles were then split and gently pulled aside to expose the target vertebra. Counting back the spines from the large vertebra Th2 until Th8 identified the target spines for the laminectomy. A big blood vein running between Th4 and Th5 (in 80\% of the rats) could also be used to locate Th5 (Figure 12). This has been used as further confirmation for counting target vertebra (de Medinaceli, 1986). After identifying the target vertebrae, laminectomy was performed at thoracic level 8 (Th8) using a bone cutter to expose the spinal cord. Care was taken not to damage the spinal cord with the rough edges of the bone after laminectomy by trimming off the rough edges. The dorsal right half of the spinal cord was transected at Th8 using a pair of micro-scissors marked at a depth of $1.25 \mathrm{~mm}$. Finally the back muscles were stitched and the skin was closed at the operation site. The 
operation site was disinfected with Brauno|ß. During the whole operation, the body temperature of the animal was maintained at $37^{\circ} \mathrm{C}$ using a heating pad. After the operation, animals were given $5 \mathrm{ml}$ of Ringer solution subcutaneously to substitute for loss of fluids during the operation. Postoperatively, an antibiotic (Cotrimoxazol; $15 \mathrm{mg} / \mathrm{kg}$ body weight) and an analgesic agent (Buprenorphine; 0,05 $\mathrm{mg} / \mathrm{kg}$ body weight) were applied for 3 days once daily. The bladder was emptied manually if necessary. Animals were taken care post-operatively as described in section 3.4.4.

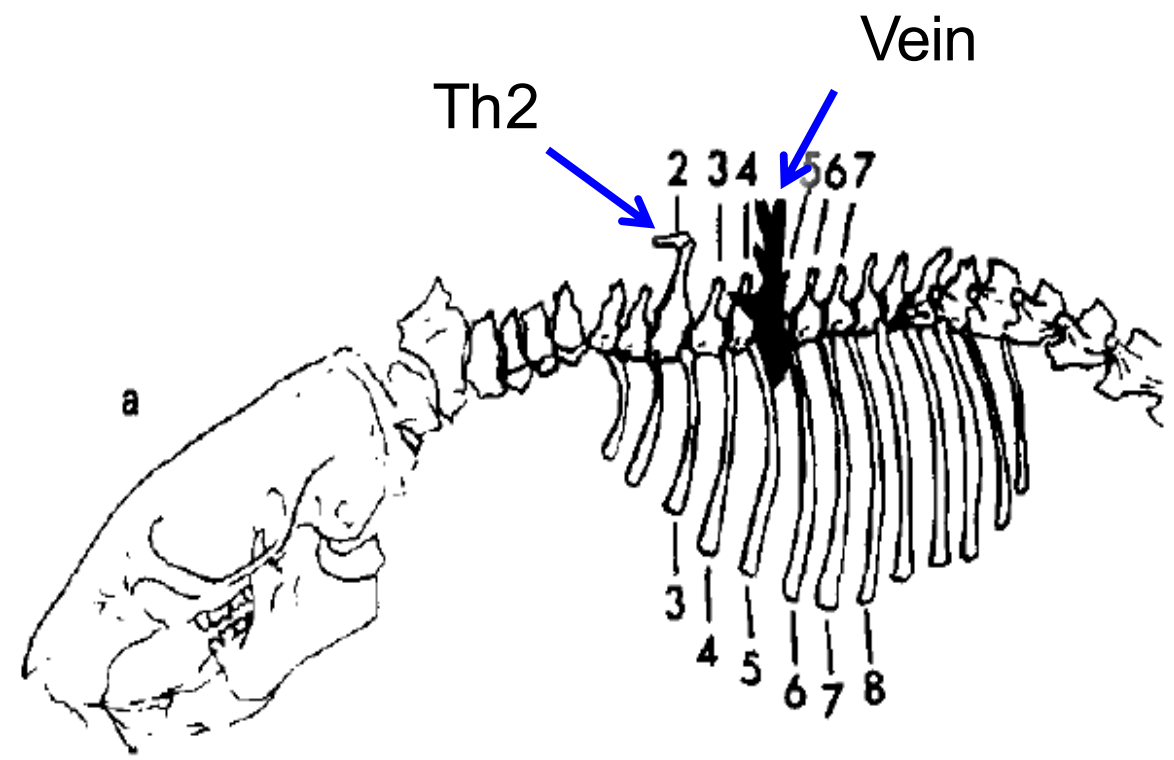

Figure 12. Rat spinal landmarks for counting the spines at thoracic level. Blue arrow located the Th2 and big blood vein running between Th4 and Th5. Figure modified from de Medinaceli, 1986.

\subsubsection{Osmotic mini-pump implantation for the anti-scarring treatment delivery to the spinal cord after dorsal hemisection}

Osmotic mini-pumps were used to continuously deliver pharmacological treatments (anti-scarring treatment) to the spinal cord lesion site. The osmotic mini-pump implantations were done in collaboration with the lab of Prof. Dr. Hans Werner Müller, Düsseldorf according to (Klapka et al., 2005). A schematic illustration of the osmotic pump implantation is given in Figure 13. In brief, osmotic mini-pumps were filled with either BPY-DCA or TRIS buffer according to manufacturer's instructions (ALZET ${ }^{\circledR}$, Cupertino, CA) before surgery. Using Isoflurane anesthesia (2-3\% in $\mathrm{O}_{2}$ and $\mathrm{NO}_{2}$ at a ratio 
of 1:2) spinal cord injury was performed as described in section 3.4.2 with minor alterations. In brief, laminectomy of vertebrae at thoracic levels 8, 9 at Th11 were performed. After right dorsal hemisection at thoracic level 8 (Th8), the dura mater was stitched with a 10/0 surgical suture to prevent leakage of CSF and the pharmacological fluid applied. One end of the catheter was inserted at Th11 and pulled to the direction of lesion via Th10 and a small piercing was made through the dura mater rostral to the lesion to insert the catheter. The catheter was placed onto the lesion through the piercing and was stabilized by stitching it to the dura mater. The other end of the catheter was connected to the osmotic mini-pump filled with the respective pharmacological treatments (anti-scarring treatment (AST) with BPY-DCA or anti-scarring buffer treatment (ASB)). Muscles and the skin were sutured on the lesion side by placing the pump on the back of the animal. $5 \mathrm{ml}$ of Ringer solution were injected subcutaneously immediately after the operation to substitute lost fluids during operation and rats were kept warm under a warming light. Postoperative care was taken as mentioned in section 3.4.4. 
a

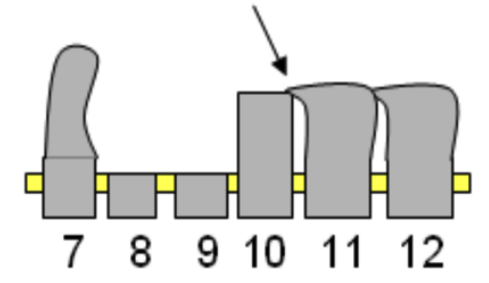

C

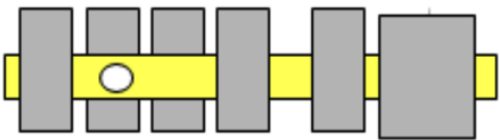

e

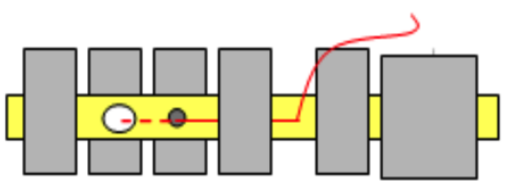

b

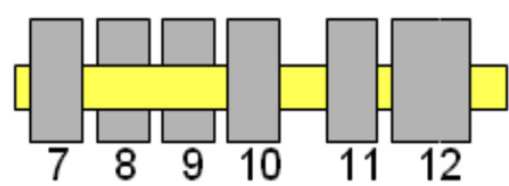

d

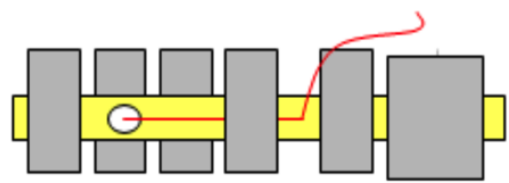

$f$

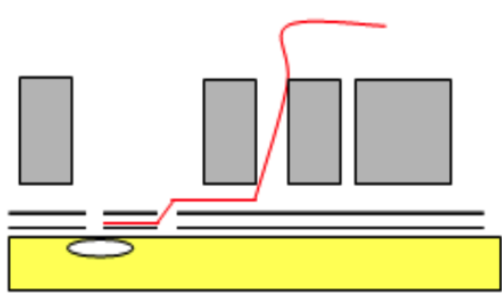

catheter

g

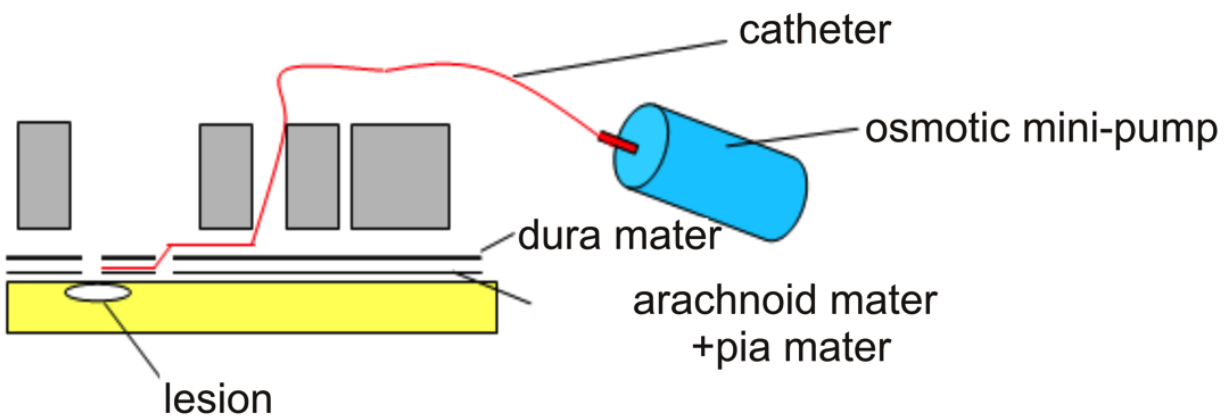

Figure 13. Schematic representations of the osmotic mini-pump implantation in the spinal cord of rats. (a) Laminectomy at thoracic vertebrae 8 and 9, and another laminectomy between vertebrae 10 and 11 (arrow), lateral view. (b) Laminectomy of the vertebrae 8, 9 and part of the vertebrae 11, top view. (c) Spinal cord lesion at Th8. (d) Insertion of the catheter between the vertebrae 10 and 11. (e) $A$ hole was made approximately $5 \mathrm{~mm}$ away from the lesion in the outer meninges (dura mater) from where the catheter intrathecally runs to the lesion site beneath the dura mater. (f) Lateral view of the catheter above and below the dura mater. (g) After inserting the catheter, the osmotic minipump was connected to the catheter. Image was adapted from Schiwy, 2010. 


\subsubsection{Pre- and post-operative care of the animals}

All animals were given Metamizole (Metapyrin ${ }^{\circledR} 1.33 \mathrm{mg} / \mathrm{ml}$ ) in drinking water 2 days pre-operation to let the animals get used to the taste of the drug. This treatment was continued for 5 days after spinal cord lesion and osmotic pump implantation in addition to the injectable analgesic. 30 minutes prior to the surgery, using Isoflurane anaesthesia, an analgesic (Buprenorphine; 0.05 $\mathrm{mg} / \mathrm{kg}$ body weight) was given subcutaneously. Immediately after the operation, $5 \mathrm{ml}$ of sterile saline water (Ringer solution) were given subcutaneously to substitute lost fluids during the operation. The animal was kept warm under a warming lamp until awake and was then returned to the home cage provided with moist food in a petridish for 3 days. Post-operation, an antibiotic (Cotrimoxazol; $15 \mathrm{mg} / \mathrm{kg}$ body weight, once a day) and an analgesic agent (Buprenorphine; $0.05 \mathrm{mg} / \mathrm{kg}$ body weight, twice a day) were applied for 3 days. The bladder was emptied manually if necessary and the animals were checked regularly for general health conditions and autophagy of the toes. If animals were found with foot lesion or toes biting, an oral treatment was given with Meloxydyl (Metacam ${ }^{\circledR} 0.5 \mathrm{mg} / \mathrm{kg}$ body weight once a day orally) and a topical wound spray applied to the affected limbs up to 57 days. Animals showed no health improvements (with swollen toes and foot) were sacrificed and eliminated from the study.

\subsubsection{Perfusions and tissue processing}

Transcardial perfusions allows uniform and rapid fixation of the tissue for the immunohistochemistry and the animals circulatory system distribute the fixative throughout body allowing preservation of tissues ultrastructure and stabilizes protein and peptide confirmation (Gage et al., 2012). For the brain and spinal cord cryosections, transcardial perfusions were performed in all animals. The animal was deeply anaesthetized by insufflation of $\mathrm{CO}_{2}$. Immediately after cessation of breathing, the animal was fixed upside down on a rack and a $\sim 10 \mathrm{~cm}$ long cut along the sternum was made. The diaphragm was cut laterally on both the sides towards the head across ribs and in parallel to the lungs. A small pair of rat tooth forceps was used to grasp the ventral tip of the heart and the left ventricle was pierced with a 
scalpel. Then the cannula was inserted in to the slit and directed up through the left ventricle into the ascending aorta. Clamping the aorta with mosquito hemostat forceps stabilized the cannula. With the aid of a peristaltic pump cold PBS ( $\mathrm{pH}$ 7.4) was injected through the cannula to flush out the circulatory blood. Once the blood flow from the nose outlet became clear or colorless (after running around $200 \mathrm{ml}$ of PBS), PBS flow was replaced by cold $4 \%$ PFA in $1 \times$ PBS ( $\mathrm{pH}$ 7.4). A successful PFA-perfusion was indicated by the stiffness of the tail and neck of the animal. The spinal cord and the brain samples were dissected carefully and post-fixed in $4 \%$ PFA overnight. The samples were then dehydrated in gradient sucrose solutions of $10 \%, 20$ $\%$ and $30 \%$ (24 hrs. in each concentration) and finally preserved at $-20^{\circ} \mathrm{C}$ until cryosectioned.

\subsection{Histology}

\subsubsection{Cryosectioning}

Following embedding the brain and the spinal cord sections in the cryomatrix, the coronal brain sections at a thickness of $30 \mu \mathrm{m}$ (Figure 14) were made at the level of RN (approximately at bregma 5.6 to 6.4, anatomy guidelines from rat brain atlas of Paxinos and Watson, 1998) to check the viral transduction at $\mathrm{RN}$ and for the quantification of RN neurons. The cryomatrix embedded spinal cord tissue was dissected into three blocks, a block of $1 \mathrm{~cm}$ diameter with the lesion in the center, a $2 \mathrm{~cm}$ cervical block (C2-C6) and a $2 \mathrm{~cm}$ thoracic block (Th1-Th5) proximal to the lesion (Figure 14). The lesion block was then cryosectioned horizontally (slice thickness $30 \mu \mathrm{m}$ ) at the level of RST. Transverse sections of cervical block (C2-C6) and thoracic block (Th1Th5) were made at a thickness of $30 \mu \mathrm{m}$ and all cryosections were dried at $37^{\circ} \mathrm{C}$ for $30 \mathrm{~min}$ and then stored at $-20^{\circ} \mathrm{C}$ until further use. 


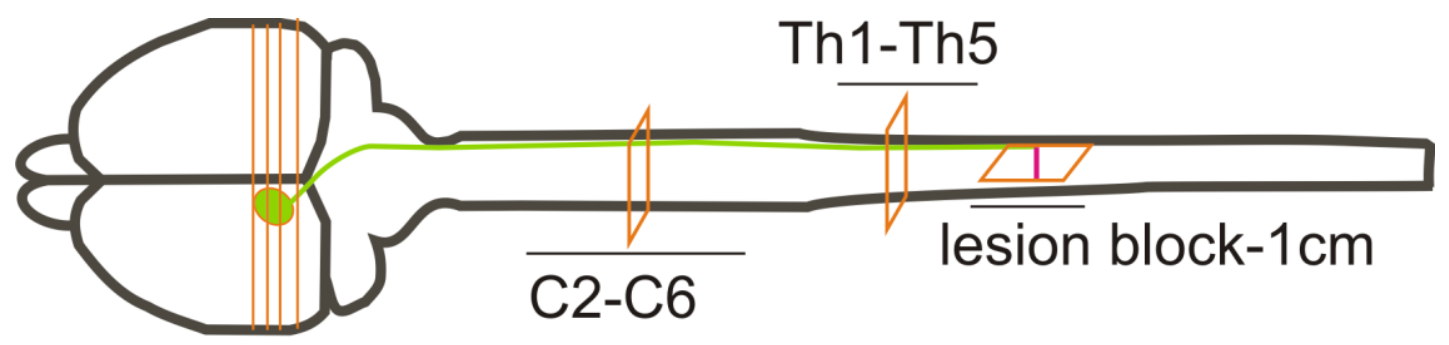

bregma 5.6 to 6.4

Figure 14. Schematic representation of the cryosectioning strategy of the brain and spinal cord. Schematic image representing the location of the EGFP-labeled RN and RST (green) coronal brain sections were made at the $R N$ level (orange lines). The lesion block of the spinal cord $(1 \mathrm{~cm})$ was cut into horizontal sections and the thoracic spinal cord (Th1-Th5) and cervical levels (C1 to C5) were cut into transverse sections. The orange squares represent the orientation of the sectioning.

\subsubsection{Immunohistochemistry}

Immunohistochemistry is a process of detecting proteins localized within a tissue by exploiting the principle of antibodies that bind specifically to the protein of interest. A secondary antibody conjugated to a fluorophore that binds specifically to the primary antibody thereby allows specific visualization of the target protein.

All specimens for immune staining were thawed at $37^{\circ} \mathrm{C}$, washed in PBS and were outlined on the slides with a Pap Pen to prevent the spilling of liquids. All immunohistological stainings were performed using the heat-mediated antigen retrieval method with citrate buffer at $\mathrm{pH} 6$ followed by blocking in $10 \%$ NGS, $1 \%$ BSA and $0.3 \%$ TritonX-100 for $1.5 \mathrm{hr}$ at room temperature (RT). Subsequently, the sections were incubated with respective primary antibodies over night at $4^{\circ} \mathrm{C}$ in a moist chamber. The spinal cord horizontal sections were incubated with polyclonal rabbit anti-GFAP antibody (1:200), polyclonal rabbit anti-GAP43 antibody (1:500). The spinal cord transverse sections were incubated with polyclonal rabbit anti-GFAP antibody (1:200), mouse monoclonal anti-NeuN antibody (1:200), rabbit polyclonal antisynaptophysin (1:1000) or chicken polyclonal anti-GFP (1:250). The midbrain sections were incubated with rabbit polyclonal anti-BAG1 antibody (1:250), goat polyclonal anti-ROCK2 antibody (1:50) and mouse monoclonal 
anti-NeuN antibody $(1: 200)$. All the sections were then incubated with respective secondary antibodies coupled to Cy3 (1:500) or Cy5 (1:250) or Alexa Fluor ${ }^{\circledR} 488$ for $1 \mathrm{hr}$ at RT (please refer to Table $3 \& 4$ for the list of the antibodies used). After further washing thrice with PBS, sections were counter stained with DAPI (4',6-Diamidino-2-phenylindole) for nuclear labeling. All the sections were air-dried, mounted with Mowiol and were stored at $4^{\circ} \mathrm{C}$ for the microscopic imaging.

\subsection{Data analysis and quantifications}

\subsubsection{NeuN counting for $R \mathbf{N}$ cell survival quantification}

For the RN neuron number and soma size quantification, midbrain sections were immunolabelled with anti-NeuN antibody for consistent labeling of the atrophied RN neurons after axotomy, as shown by (Kwon et al., 2002b). The $\mathrm{RN}$ was identified by the distinct morphology of magnocellular RN neurons with the adjacent midbrain structures of the oculomotor nerve fibers and the substantia nigra as anatomical references (Carter et al., 2011). Three of the brain sections per animal at the level of the $\mathrm{RN}$ were used for quantification. Images were sampled using a fluorescence microscope (AxioPlan equipped with a CCD camera and MosaiX software, Zeiss, Goettingen, Germany) using a $10 \times$ optical lens. The number and size of NeuN-positive cells with clear DAPI nuclei were quantified at the magnocellular portion of the RN using AxioVision LE software. NeuN quantifications were made in the injected RN (lesioned RST) and uninjected RN (unlesioned RST), which served as internal control in each animal. $\mathrm{RN}$ neurons were classified according to their size. The comparisons among the groups were always made with AAV.BAG1 vs. AAV.EGFP and AAV.sh.ROCK2 vs. AAV.sh.firefly as respective control groups.

\subsubsection{Quantification of axonal sprouting}

Sprouting was defined as projections or branches of RST axons towards the gray matter (Liu et al., 1999; Steward et al., 2003). Axonal sprouting for the 
pilot experiment (section 3.3.1) was quantified in the spinal cord horizontal sections at the level of the RST. A total of 3 to 4 sections per animal were analyzed and quantification was performed at $2 \mathrm{~mm}$ proximal to the lesion. The length of EGFP-labeled rubrospinal axons spreading on to the gray matter was measured using NeuronJ plug-in of ImageJ software $(\mathrm{NIH}$, Maryland, USA) (Meijering et al., 2004). The quantification was normalized to the number of EGFP-labeled axons counted at $2 \mathrm{~mm}$ proximal to the lesion in each animal to avoid the transduction variation bias among the animals and the comparisons were done among all the groups. For the experimental setup evaluating gene therapy with AAV.BAG1, AAV.reggie1, AAV.miRNA-134 and AAV.sh.ROCK2 (Table 3.3.3), the quantification of the axonal sprouting was performed in the spinal cord transverse sections at cervical (C2-C6) and thoracic levels (Th1-Th5) proximal to the lesion. Digital images were constructed using AxioVision MosaiX software (Zeiss, Göttingen, Germany) at $10 \times$ magnification. An Axioplan microscope equipped with an Apotome module was used to visualize the axonal sprouting with higher magnification. A total of 5 sections covering each level of the sectioned spinal cord were used for the quantification. Axonal sprouting was quantified by counting the EGFP-positive axonal sprouts that extended from the RST bundle on to the gray matter at defined distances (Rexed's laminae $\mathrm{V}$ to VIII) until the central canal (Figure 28). In order to avoid bias by viral transduction variability among animals, an axonal sprouting index was determined by normalization of sprouts to the total EGFP-positive axon number counted at high cervical level (C2) in each animal.

\subsubsection{Quantification of lesion size and axonal degeneration}

Lesion quantification for the pilot experiment (section 3.3.1) was done in a total of 3 to 4 horizontal spinal cord sections counterstained with DAPI. We could not use an additional immunolabel for these sections against GFAP due to multiple native fluorescences derived from the AAV-tracing of the RST (with EGFP) and CST (with DsRed) and further immunostaining against GAP43 with Cy5-conjugated antibody. Therefore, we evaluated the lesion size mainly based on the tissue morphology and the strongly DAPI-positive 
area indicating an accumulation of cells (glial or inflammatory cells) around the lesion in general. The lesion area was measured using ImageJ software $(\mathrm{NIH}$, Maryland, USA). For lesion quantification in the experimental set-up of gene therapy with AAV.BAG1, AAV.reggie1, AAV.miRNA-134 and AAV.sh.ROCK2 (section 3.3.3) a total of 4 horizontal spinal cord sections at RST level (60 $\mu \mathrm{m}$ apart) were immunostained against GFAP to define the lesion area. Digital images were constructed using AxioVision MosaiX software or AxioVision Panorama ${ }^{\mathrm{TM}}$ software (Axioplan, Zeiss, Göttingen). Areas with little or no GFAP immunoreactivity were defined as the lesion (Steward et al., 2003) and were measured using AxioVision LE software. Axonal degeneration was quantified at defined distances starting at the proximal lesion border to the EGFP-positive RST axon bundle. Again, the quantification was normalized to total EGFP-labeled RST axons in order to avoid bias due to viral transduction variability.

\subsection{Behavioral studies}

\subsubsection{BBB - open field test}

All behavioral experiments were performed in a blinded fashion. BBB is one of the fundamental tests for the evaluation of spinal cord lesion models and it allows the analysis of various foot parameters with the score points from 0 to 21 ("0" means no observable hind limb movements and "21" means the hind limb has consistent plantar stepping, coordination, toe clearance, trunk stability and parallel paw placement and the tail is always up). The motor behavior analysis for the BBB was performed according to (Basso et al., 1995) where the animals were allowed to walk freely for 4 minutes in an open field box made of Plexiglas $(75 \times 30 \mathrm{~cm})$. The observations of the foot parameters were scored manually for both hind limbs (RHL-lesioned and LHL-unlesioned). A basic score was taken before the $\mathrm{SCl}$ and after the $\mathrm{SCI}$ scores were collected at regular time points (Figure $7 b$ \& 10b). In general, BBB is a broad functional test for complete SCI models. In our test, being a partial lesion model, to increase the sensitivity of the BBB test, we assessed some specific foot parameters such as toe clearance, predominant paw position and trunk stability as a sub score. 


\subsubsection{Horizontal ladder rung test}

The ladder rung test is used to analyse the motor function both quantitatively and qualitatively (Metz and Whishaw, 2002, 2009). Initially, the rats were trained for two weeks to get used to walk on a $1 \mathrm{~m}$ horizontal ladder separated by metal rungs. After the training, a ladder rung test was performed for 13 weeks after the lesion and animals were videotaped with a fixed video camcorder (Figure 7b). Each animal was given 5 runs for each trial. From trial to trial, by varying the space between the rungs, the rung pattern was changed to prevent the animals from getting used to a certain rung pattern. The qualitative measurement by the foot fault scoring normally differentiates the placement of fore- and hind limbs in six different scores, but in the model which is used here, only the hind limbs ( $\mathrm{HL}$ ) were taken into account and only three scores were counted as miss steps: "Total miss" is defined as the hind limb falling between the rungs and leading to posture and balance disturbance of the animal, the "deep slip" is defined as the limb touching the rung but not holding the weight which causes a fall of the rat, and the "slight slip" is defined as a placing of the limb on the rung and slipping off but not resulting in a fall of the rat (Figure 15).

All the video recordings were analysed and the missed steps were counted in a frame-by-frame slow motion. For statistical analysis, the error rate was counted in five runs per animal (errors/step) and the average calculated for the right hind limb (RHL, lesioned). The difference of the RHL score among the groups from basic to several weeks was used for the statistical analysis. 


\section{Foot Fault Score}
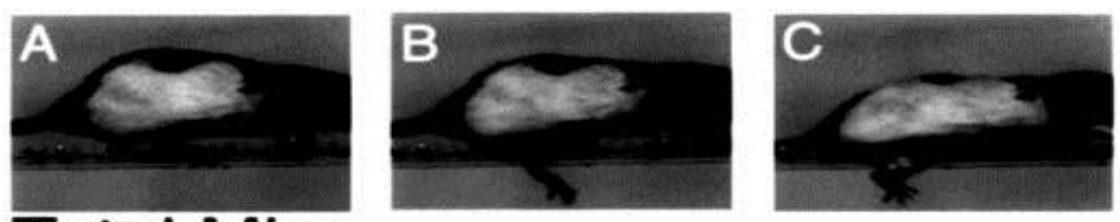

\section{Total Miss}
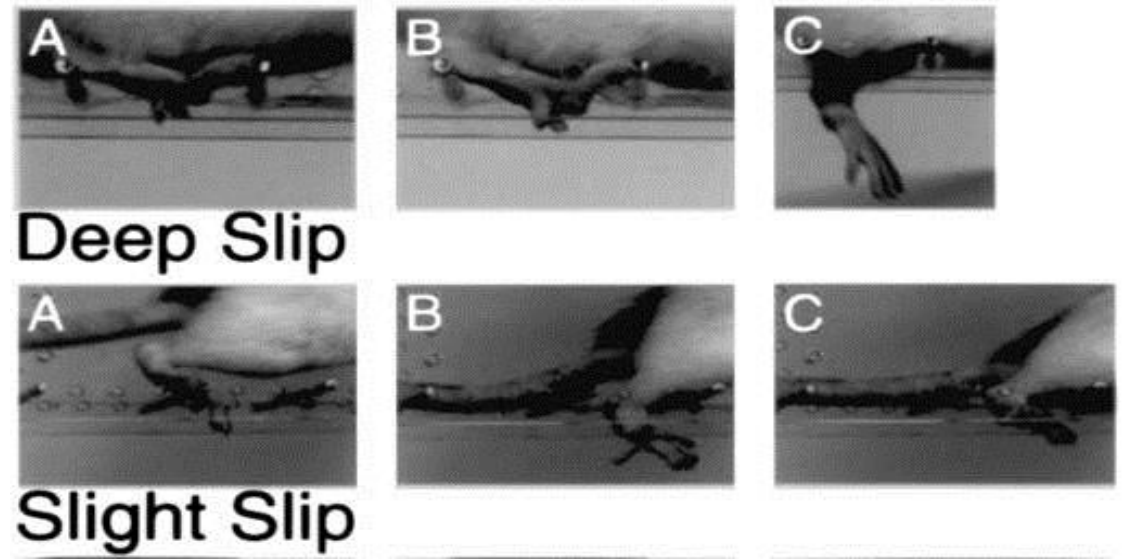

Figure 15. Horizontal ladder rung test. Consecutive photographs illustrating the categories of foot falls: total miss, deep slip and slight slip during the ladder-rung walk. Image was modified from Metz and Whishaw, 2002.

\subsubsection{CatWalk ${ }^{T M}$ gait analysis}

In order to evaluate the gait of the animals more precisely, a CatWalk ${ }^{\mathrm{TM}}$ analysis was performed using a CatWalk ${ }^{\mathrm{TM}}$ apparatus (Noldus, Weiningen, Netherlands) according to (Hamers et al., 2001). Before the SCl, all animals were trained for 2 weeks on every alternate day to run on the CatWalk ${ }^{\mathrm{TM}}$ apparatus. A basic score was taken once the animals were able to perform 3 flawless runs on the walkway. Animals were allowed to run on the CatWalk ${ }^{\mathrm{TM}}$ glass floor $(90 \times 10 \mathrm{~cm})$ and the refractions of the illuminated footprints on the glass floor were captured by a video camera below the runway. After the lesion, the score was assessed at regular time points (Figure 10b). The CatWalk ${ }^{\mathrm{TM}}$ XT9 Software was used for foot print classification and analysis of various parameters such as base of support, regularity index, print area and maximum intensity (paw pressure). 


\subsection{Statistics}

Multiple group comparisons were analyzed by one-way ANOVA followed by Tukey's post-hoc test and two group comparisons were performed by an independent $t$-test. For comparing one variable with others within the group one-way ANOVA followed by Dunnett's test was performed. All the statistics were performed using SPSS Statistics V.21 (BMM, Armonk, NY). Data are represented as mean \pm SEM as indicated. Significances are indicated with ${ }^{*} p<0.05 ;{ }^{* *} p<0.01 ;{ }^{* * *} p<0.001$. 


\section{Results}

\subsection{Pilot project: Combination treatment with AAV.BAG1 and BPY-DCA for 16 weeks after dorsal spinal cord hemisection in rats}

\subsubsection{Viral injections and spinal cord lesion}

To demonstrate sufficient transduction of the red nucleus, cortex and the respective RST and CST and to assure the expected extent of the lesion, animals were stereotactically injected with AAV vectors and three weeks later a dorsal spinal cord hemisection was performed at Th8. Additionally, animals were subjected to osmotic pump implantation immediately after lesion to apply the AST with BPY-DCA (Figure 7 and Table 6). The cortical injections with AAV.DsRed were used in this study to evaluate if any compensatory spontaneous axonal sprouting from CST towards the RST rostral to the SC lesion could be observed. Sixteen weeks after the dorsal hemisection the animals were perfused. Analysis of the EGFP and DsRed fluorophores expressed by the respective AAV vectors could visualize that the stereotactically injected AAV successfully transduced a defined area of the midbrain including the RN (Figure 16a) and the sensorimotor cortex (Figure 16b) as well as the projecting axons in the contralateral RST (EGFP, green) and CST (DsRed, red) (Figure 16c\&d). The ipsilateral RST was also partly labeled in addition to the contralateral (lesioned) RST as some of the RN axonal projections are known to descend ipsilaterally in the dorsolateral funiculus of the spinal cord (Küchler et al., 2002). Additionally, this could be correlated to a wider spread of the virus around the injection site in the $\mathrm{RN}$ in some animals. The right dorsal hemisection resulted in a complete transection of the RST and CST (Figure 16d).

In the pilot project, several animals were found to have severe foot lesions due to autophagy and licking of the toes of the non-lesioned hind limb. This self-mutilating behavior was reported in rodents after experimental spinal cord lesions and could be due to a dysfunction of the gamma-aminobutyric acid class B (GABA-B) system and affection of sensory inputs after the spinal cord lesion (Rahimi-Movaghar; Yezierski, 1996; Zhang et al., 2001). Animals with severe foot lesions (swollen feet and toes) even after treatment with 
topical antiseptic wound spray and with Meloxidyl were sacrificed and excluded from the study. In addition to this, some AAV injections in the RN were not successful and the injections were found to be above the RN and failed to label the RN and subsequently the RST. These animals with very low or no RST transductions were also eliminated from the study (see section 3.3.1). Due to the low number of remaining animals a statistical conclusion could not be drawn in this pilot experiment. 

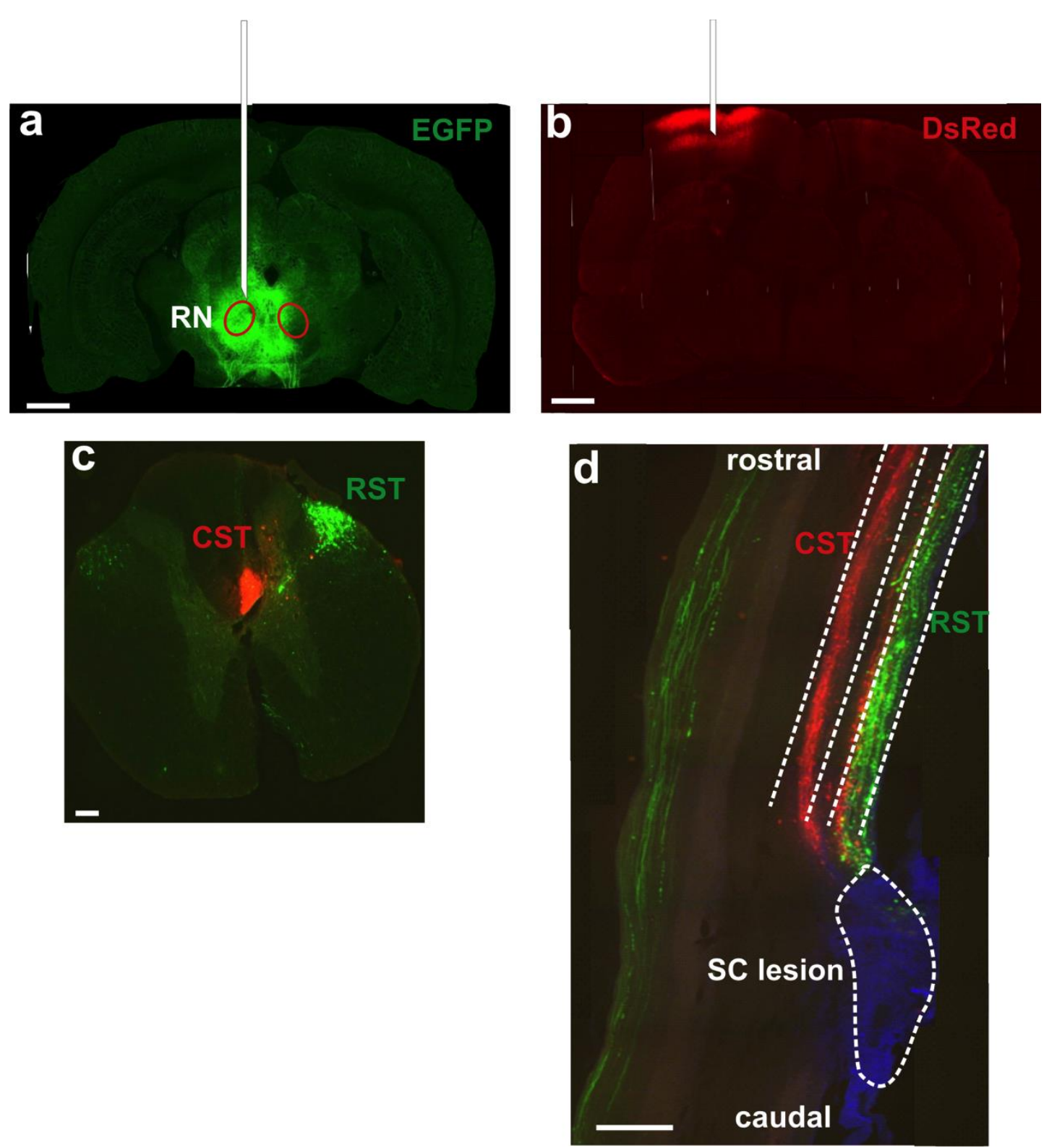

Figure 16. Experimental paradigm for combination treatment with AAV.BAG1 and AST. (a) Representative coronal brain section showing $A A V$-mediated EGFP-expression (green) at 16 weeks after AAV injection to the ipsilateral RN. Scale bar $1000 \mu m$. (b) Representative coronal brain section showing AAV-mediated DsRed-expression (red) at 3 weeks after $A A V$ injection to the ipsilateral sensorimotor cortex. Scale bar $1000 \mu \mathrm{m}$. (c) Representative transverse spinal cord section showing anterograde labeling of RST by AAV-mediated EGFP expression and CST by AAV-mediated DsRed-expression. (d) Representative longitudinal spinal cord section showing anterograde labeling of RST by AAV-mediated EGFP-expression and CST by AAV-mediated DsRed-expression and DAPI (blue). White dashed lines mark the lesion. Scale bar $500 \mu m$. AAV= adeno-associated virus; $R N=$ red nucleus; $C X=$ cortex; $R S T=$ rubrospinal tract; $S C l=$ spinal cord injury; $C S T=$ cortico spinal tract. 


\subsubsection{Effect of combination treatment with AAV.BAG1 and AST on axonal sprouting at 16 weeks after dorsal spinal cord hemisection}

We addressed the question if the anti-scarring treatment (AST) with BPYDCA in combination with the anti-apoptotic treatment with AAV.BAG1 would be beneficial for axonal regeneration, sprouting and functional motor behavior after $\mathrm{SCl}$. The experiment was performed as described in section 4.1.1. For quantification of axonal sprouting, a total of 3 to 4 horizontal spinal cord sections per animal at the level of RST and CST were analyzed. EGFPlabeled axonal sprouts extending into the gray matter of the spinal cord $2 \mathrm{~mm}$ rostral to the SC lesion were identified (Figure 17a) and the length of the axonal sprouts was measured using NeuronJ plug in of ImageJ software ( $\mathrm{NIH}$, Maryland, USA) (Meijering et al., 2004). Axonal sprouting was normalized to the number of EGFP-positive axons in each animal to avoid a bias due to variations in the transduction efficiencies among the animals. We found a trend to more axonal sprouting in the AAV.BAG1 treated groups either alone or in combination with the anti-scarring treatment (AST) or antiscarring buffer (ASB) (Figure 17b). Anti-scarring treatment alone did not show much influence on the axonal sprouting and we did not find any CST axons sprouting caudal to the lesion. The CTB injections in the RN were unable to label the regenerating RST axons after the SC lesion. We found that the distribution of the CTB tracer was minimal in the brain tissue and possibly the atrophied $\mathrm{RN}$ neurons were unable to take up the dye after the lesion. We also immunolabeled the spinal cord sections against GAP-43 to visualize any regenerating axons. However, the GAP-43 staining appeared rather unspecific and we could not identify any regenerating axons (Figure 18). Taking this outcome into consideration, GAP-43 immunolabeling was not considered useful for our quantifications of sprouting or axonal regeneration. Due to the limitations in animal numbers caused by foot lesions and unsuccessful RN injections, we could not draw robust statistical conclusions about the combination of AAV.BAG1 and AST with BPY-DCA in this experiment (see section 3.3.1). 
a
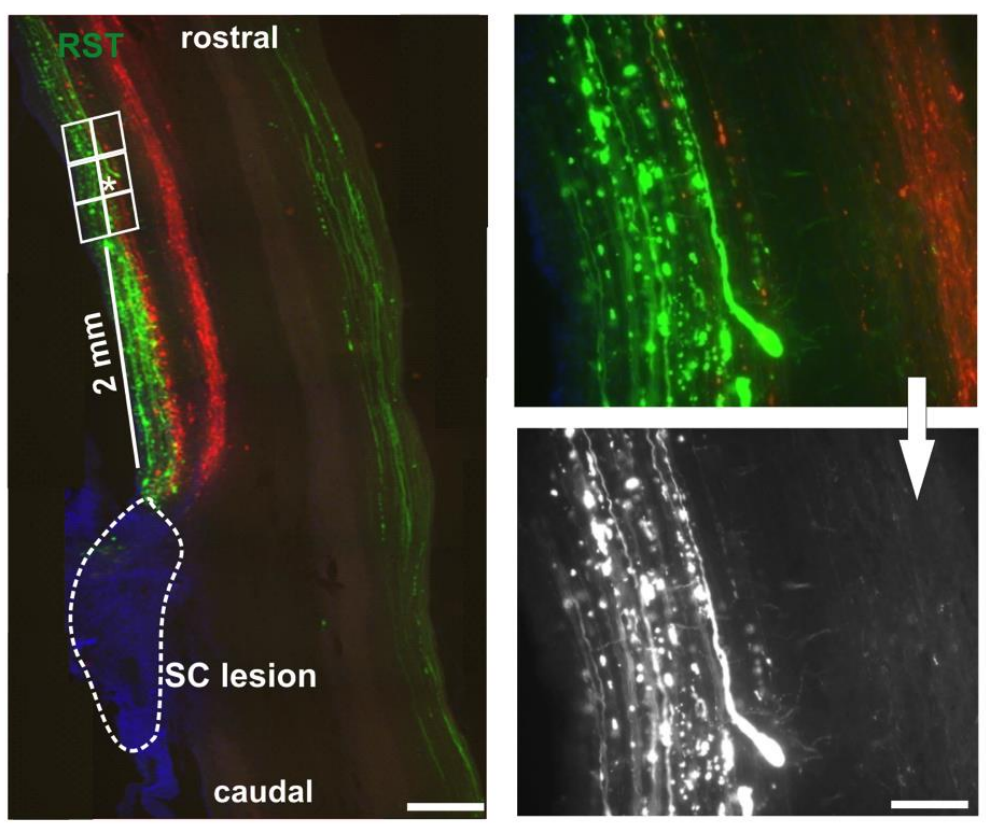

b

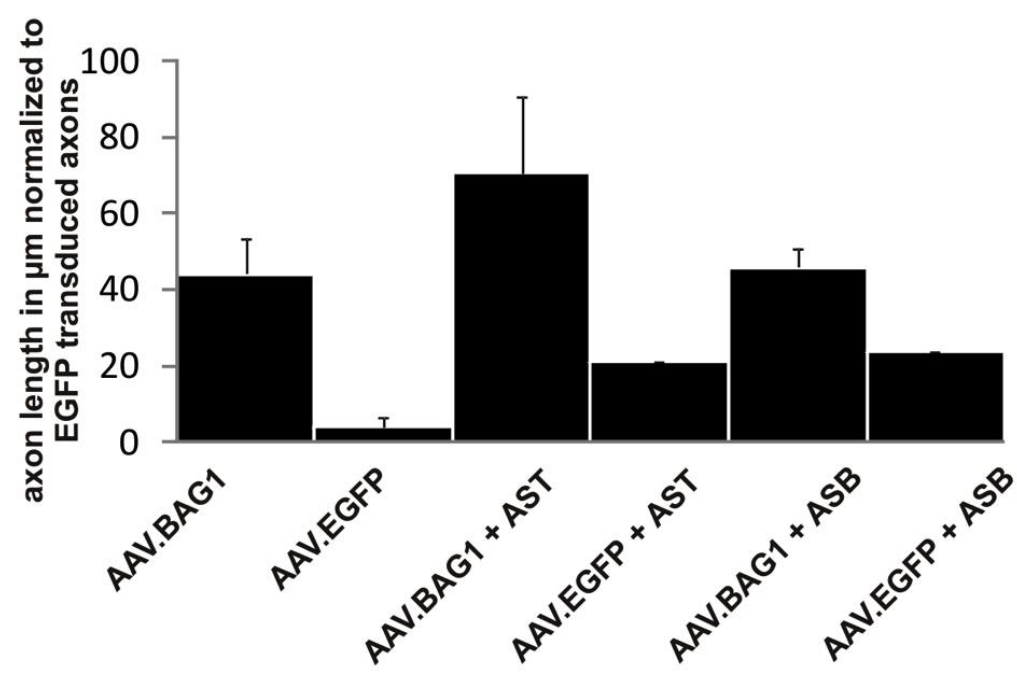

Figure 17. Effect of combination treatment with AAV.BAG1 and AST on axonal sprouting 16 weeks after dorsal spinal cord hemisection. (a) Representative longitudinal section of the spinal cord depicting the model of axonal sprouting quantification and anterograde labeling of RST with AAVEGFP (green) and CST with AAV.DsRed (red) and DAPI (blue). White dashed lines mark the lesion. Square white boxes represent the areas used for quantification of axonal sprouting $2 \mathrm{~mm}$ rostral to the lesion. The higher magnification of one square (marked with a star) is shown as fluorescent and grayscale image (for quantification in ImageJ). Scale bar horizontal spinal cord $500 \mu \mathrm{m}$; higher magnification image, $100 \mu \mathrm{m}$. (b) Axonal sprouting quantification. Bars represent the means \pm SEM. AAV.BAG1, $n=3$; AAV.EGFP, $n=2 ; \quad A A V . B A G 1+A S T, \quad n=2 ; \quad A A V . E G F P+A S T, \quad n=1$; $A A V . B A G 1+A S B, n=2 ; A A V . E G F P+A S B, n=1$. 
In this experiment, the AAV.BAG1 treatment showed a clear trend towards more axonal sprouting compared to other treatments in spite of the limited number of animals. In addition to this, the need for a better anterogradetracing agent for the tracking of axonal regeneration after spinal cord lesion was noted for further experiments.

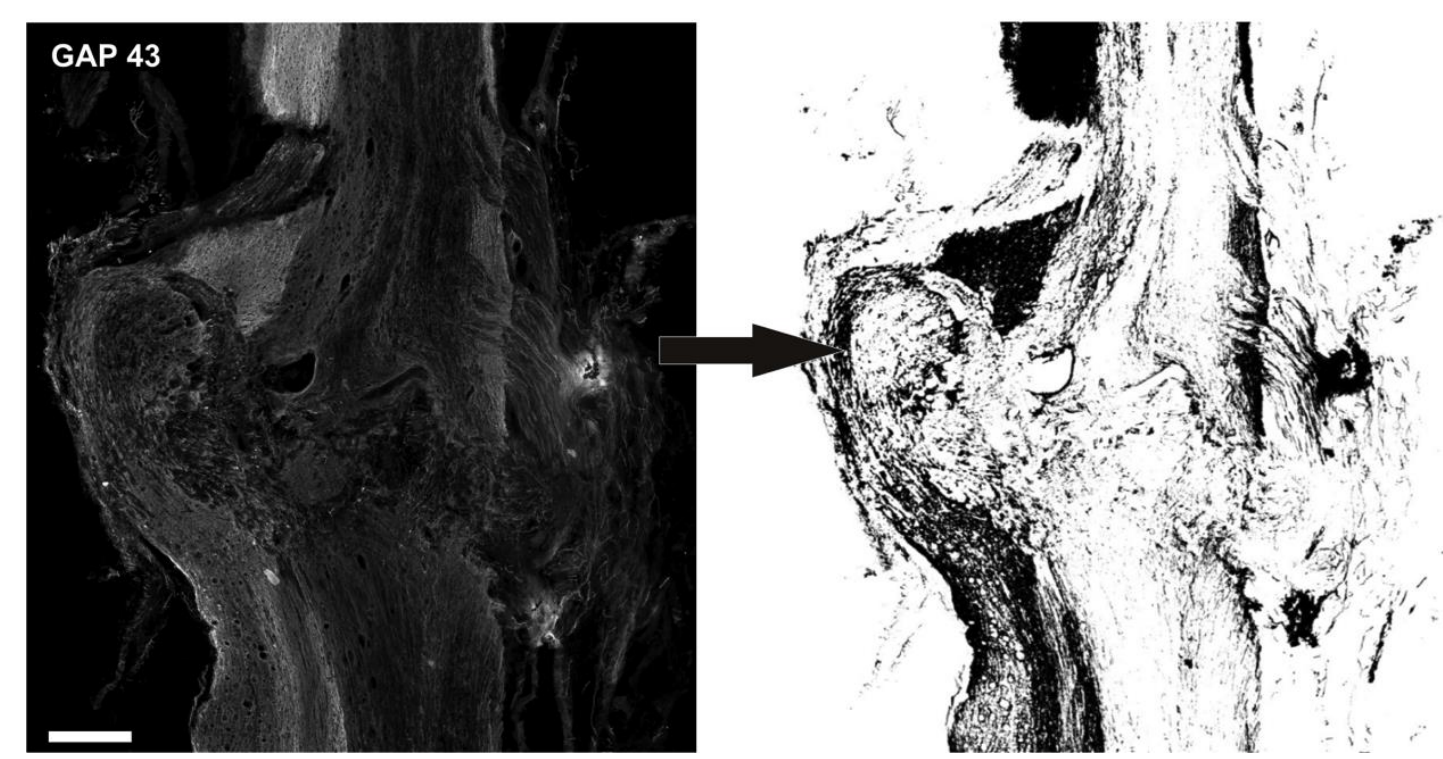

Figure 18. GAP-43 immunolabeling for visualization of regenerating RST axons at 16 weeks after dorsal spinal cord hemisection. Representative longitudinal section of the spinal cord with GAP-43 labeling (Cy5). The corresponding binary image (arrow) depicts the dark lines (axons) immunolabeled for GAP-43, but a massive unspecific staining is also observed. A clear identification of regenerating axons therefore was not considered feasible on the basis of this staining result.

\subsubsection{Osmotic mini-pump implantation resulted in increased lesion size}

\section{at 16 weeks after dorsal spinal cord hemisection}

Osmotic mini-pumps were used to deliver the antiscaring treatment (BPYDCA or TRIS buffer as control) continuously at a controlled speed and over a specific time period. This osmotic pump implantation was reported to be safe in several other models (Doucette et al., 2000; Verma et al., 2004). As this osmotic mini-pump was used for the first time in our experimental paradigm, the effect of osmotic mini-pump implantation on the lesion size was analyzed. 
A total of 3 to 4 spinal cord horizontal sections per animal were subjected to a DAPI counter stain for nuclear staining. Due to multiple native colors from the AAV tracing of the RST (EGFP) and CST (DsRed) and further immunostaining of GAP-43 with Cy5 conjugated antibody, we could not additionally stain the sections with GFAP for determination of lesion size. Therefore, in this pilot experiment the lesion quantification was performed based on tissue morphology and DAPI staining. The area of $1 \mathrm{~cm}$ around the lesion was photographed with AxioVision Panorama ${ }^{\mathrm{TM}}$ software (Axioplan, Zeiss, Göttingen). The lesion area was then quantified with ImageJ software $(\mathrm{NIH}$, Maryland, USA). We found that the animals implanted with the osmotic mini-pump (AAV.BAG1+AST, AAV.EGFP+AST, AAV.BAG1+ASB \& AAV.EGFP+ASB) had a bigger lesion size (2 to 5 fold) compared to the animals without pump implantation (AAV.BAG1 and AAV.EGFP) independent of the applied pharmacological treatment (Figure 19a \&19b). A similar effect had been reported in mice with brain infusions using osmotic mini-pump implantation (Jabłońska et al., 1993). Formation of scar tissue around the catheter of the osmotic mini pump was observed at the lesion site, which might lead to larger lesion sizes in our experimental paradigm.

This data shows that in this experimental paradigm the implantation of osmotic mini-pumps led to an increased lesion size compared to the animal groups without pump implantations. Therefore, the mini-pump paradigm appears not suitable for the analysis of therapeutic drug effects in this particular spinal cord lesion model. 
a

AAV.BAG1

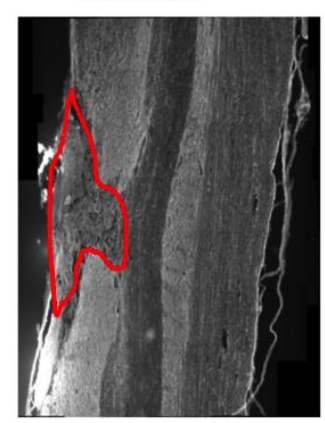

AAV.EGFP

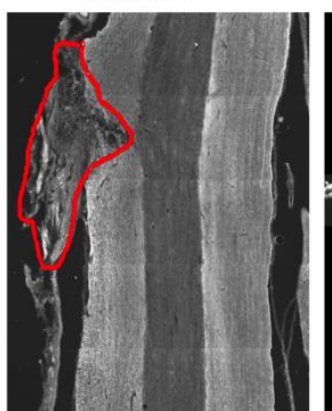

AAV.BAG1+ AST

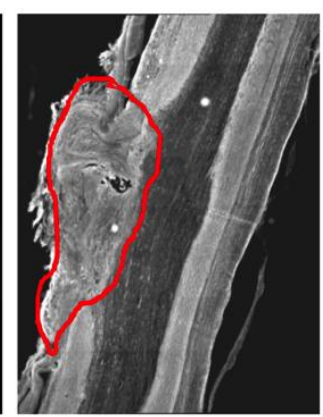

AAV.EGFP+AST

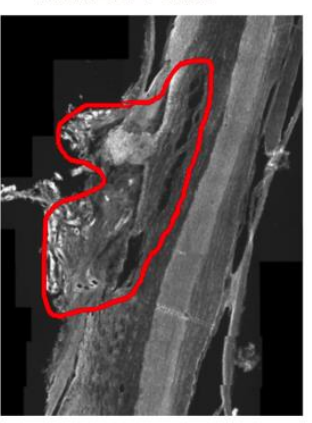

AAV.BAG1+ ASB

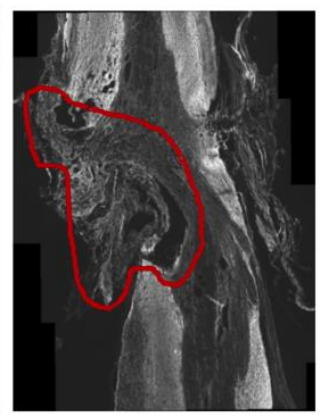

AAV.EGFP+ASB

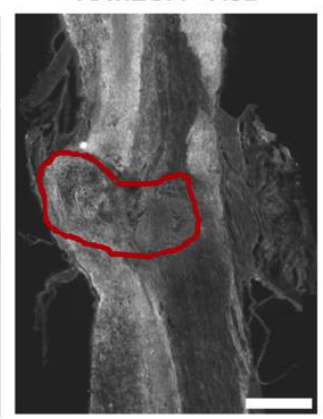

b

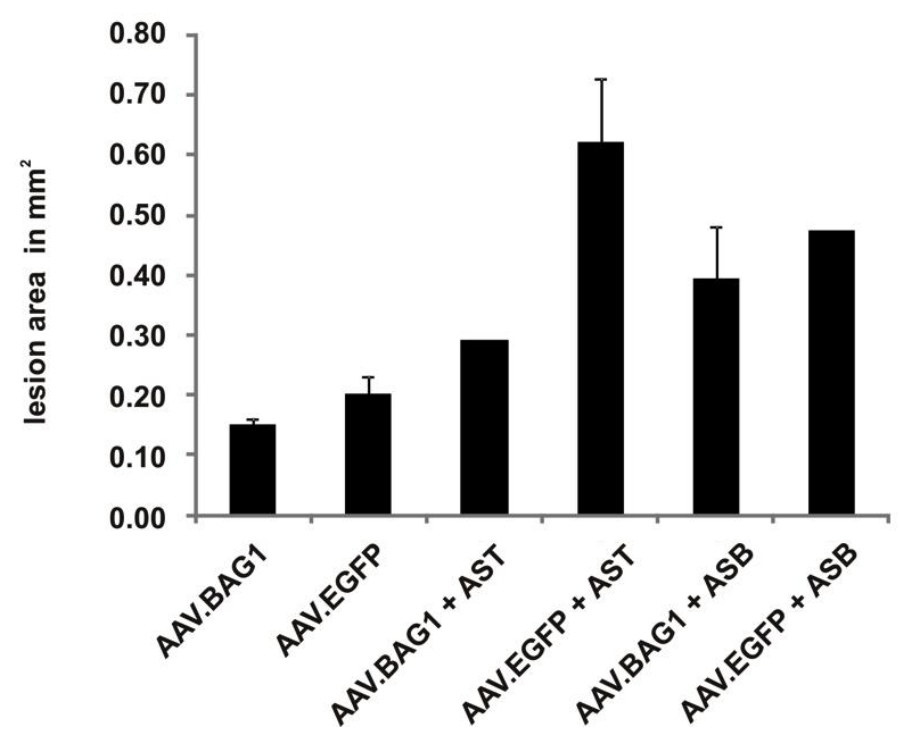

Figure 19. Effects of osmotic mini-pump implantation on lesion size at 16 weeks after dorsal spinal cord hemisection. (a) Representative photomicrographs of longitudinal SC sections of animals treated with AAV.BAG1, AAV.EGFP, AAV.BAG1+AST, AAV.EGFP+AST, $A A V . B A G 1+A S B, A A V . E G F P+A S B$. The red highlighted area represents the lesion. Scale bar $500 \mu \mathrm{m}$. (b) Lesion size quantification. Bars represent the means \pm SEM. AAV.BAG1, $n=3 ;$ AAV.EGFP, $n=2 ; A A V . B A G 1+A S T, n=2$; $A A V . E G F P+A S T, n=1 ; A A V . B A G 1+A S B, n=2 ; A A V . E G F P+A S B, n=1$. 


\subsubsection{Effect of combination treatment with AAV.BAG1 and AST on motor behavior at 16 weeks after dorsal spinal cord hemisection.}

\subsubsection{BBB}

In order to investigate the effect of combination treatment with AAV.BAG1 and AST (BPY-DCA) on motor recovery, a BBB test was done according to (Basso et al., 1995). The animals were allowed to walk in an open field for 4 minutes and the basic score was taken. The score was obtained every alternate week up to 13 weeks after the spinal cord lesion (Figure 7b). At the basic level, all the animals scored a total 21-point BBB score. The score was not different among the groups even after the viral injections, indicating that the viral injections did not have a negative effect on the motor behavior. At

week 1 after right dorsal spinal cord hemisection the score went down to 13 in all groups indicating a successful partial lesion. Over time, the score improved up to 18 by 13 weeks in all groups, but no significant differences among the groups in the BBB total score were observed (Figure 20a).

\subsubsection{Horizontal ladder rung test}

The horizontal ladder rung test was used to qualitatively and quantitatively analyze the gait of rats after dorsal spinal cord hemisection. Rats were trained for 2 weeks on the ladder rung before the basic score was taken. After the spinal cord lesion, a score was taken at week 1 which was followed up to week 13 (Figure 7 b). All tests were videotaped and analyzed manually with a frame-by-frame analysis. An average of 5 runs was taken for each test per animal and the missed steps were quantified for the affected right hind limb $(\mathrm{RHL})$. All the rats were able to cross the ladder rung without any errors before SCI but one week after SCl, significant errors were noted for the RHL. Over the weeks, the number of missed steps and errors was reduced, but no significant differences were seen among the groups (Figure 20b). 
a

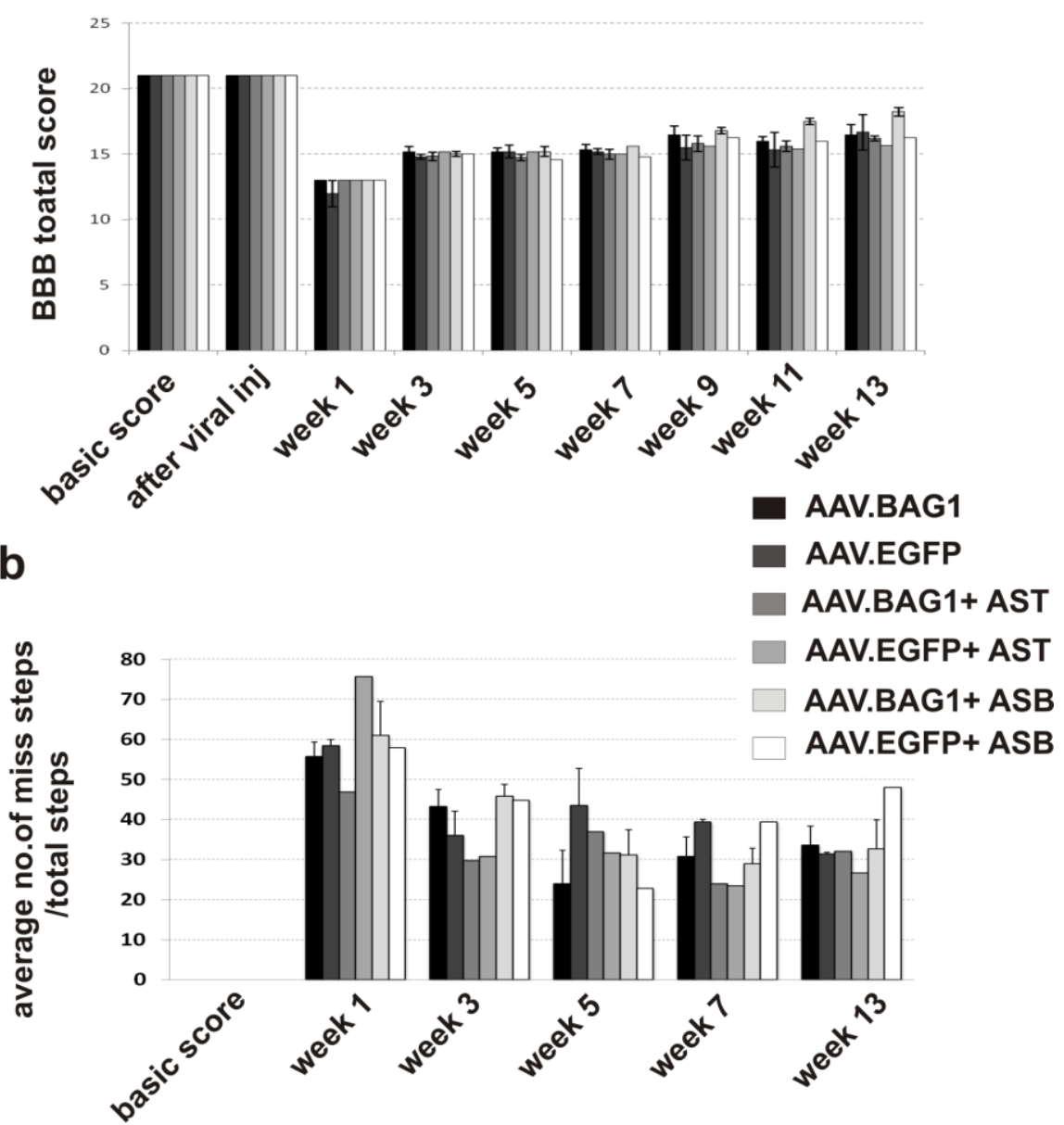

Figure 20. Effect of combination treatment with AAV.BAG1 and AST on motor behavior at 16 weeks after dorsal spinal cord hemisection. (a) Quantification of BBB total score (21 point scale) of the right hind limb measured before the lesion and 1, 3, 5, 7, 9, 11 \& 13 weeks after RST lesion. (b) Quantification of horizontal ladder rung test score as average of miss steps of the right hind limb measured before the lesion and 1, 3, 5, 7 \& 13 weeks after RST lesion. Bars represents the means \pm SEM. AAV.BAG1, $n=3$; AAV.EGFP, $\quad n=2 ; \quad A A V . B A G 1+A S T, \quad n=2 ; \quad A A V . E G F P+A S T, \quad n=1$; $A A V . B A G 1+A S B, n=2 ; A A V . E G F P+A S B, n=1$.

Taken together, both BBB and horizontal ladder rung test did not show any significant differences in motor skill improvement in any of the treatment groups after SCl. 


\subsection{Anterograde tracing of RST after dorsal spinal cord hemisection}

4.2.1 Anterograde tracing of the RST with AAV.EGFP before and BDA or AAV.DsRed after the right dorsal spinal cord hemisection

As described in the results section of our pilot experiments, no regenerating rubrospinal axons after the spinal cord lesion using CTB labeling could be identified. Therefore we investigated other anterograde tracing methods in two different sets of experiments (Table $7 \& 8$, Figure $8 \& 9$ ). In the first set of experiments (Table $7 \&$ Figure 8 ), the animals were first injected into the RN with AAV.EGFP to trace the RST. After week 3 (to allow for sufficient AAV transduction and transgene expression), the animals were subjected to right dorsal spinal cord hemisection. Another 3 weeks after the dorsal hemisection of the spinal cord, a second injection into the $\mathrm{RN}$ was performed with BDA or AAV.DsRed to specifically label the rubrospinal axons after the lesion. Four weeks after the second $\mathrm{RN}$ injection, animals were perfused and the spinal cord and the brain sections were analyzed for BDA, EGFP and DsRed transduction. The first stereotactic RN injections with AAV.EGFP could sufficiently transduce the $\mathrm{RN}$ in the midbrain and subsequently its axonal projections in the RST running contralaterally in the spinal cord. Interestingly, the second stereotactic RN injection with BDA could be seen in the RN, while we could not detect a labeling of the RST even at high cervical levels (Figure 21). The second injection with AAV.DsRed on the other hand, did label RN neurons and a few axonal projections of the RST in the contralateral SC at the cranial and cervical levels but did not sufficiently label the axons more distal of the cervical level until the lesion site (Figure 22). 

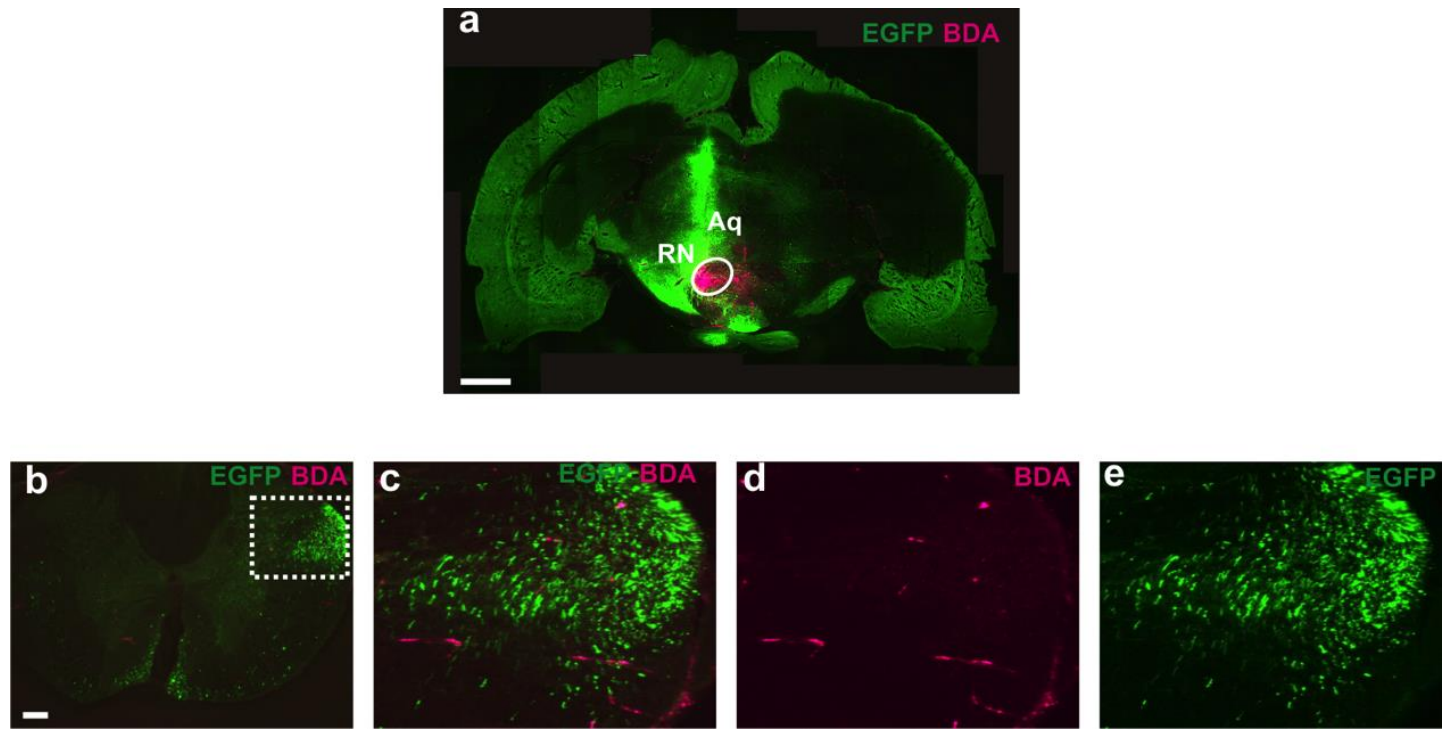

Figure 21. Anterograde tracing of the RST with AAV.EGFP before and BDA after dorsal spinal cord hemisection, respectively. (a) Representative coronal midbrain section showing $R N$ injection with $A A V$ mediated EGFP-expression (green) and BDA (pink). AAV.EGFP was injected before and BDA after dorsal hemisection, respectively. Scale bar $1000 \mu \mathrm{m}$. $R N=$ red nucleus, $A q=A q u e d u c t$. (b) Representative transverse cervical spinal cord section showing RST tracing with EGFP (green) and BDA (pink). Scale bar $200 \mu \mathrm{m}$. Corresponding photomicrographs $\boldsymbol{c}, \boldsymbol{d} \&$ e show a higher magnification of the RST (white dotted line square box in $\boldsymbol{b}$ ). 


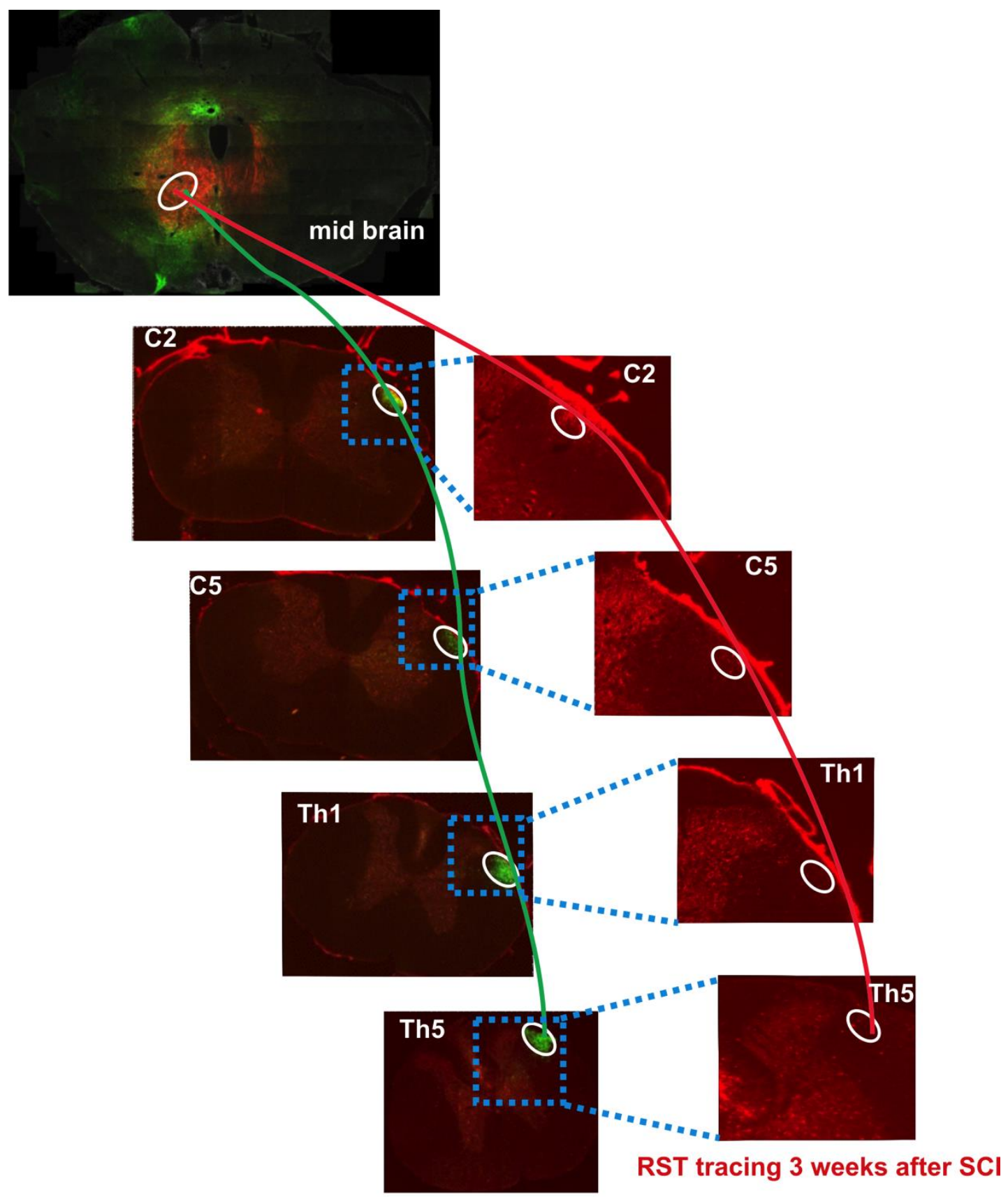

RST tracing before SCI

Figure 22. Anterograde tracing of RST with AAV.EGFP and AAV.DsRed before and after dorsal spinal cord hemisection, respectively. Representative coronal midbrain section showing RN injection with AAVEGFP (green) before and AAV.DsRed (red) after dorsal hemisection, respectively. The green line depicts the path of the RST (white oval shape) visualized with EGFP at C2, C5, Th1 and Th5 proximal to the lesion. The RST is additionally stained by AAV.DsRed (dotted blue square of the corresponding spinal level) shoun in higher magnification, where the red line narrates the path of RST at 3 weeks after dorsal hemisection. $R N=$ red nucleus; RST= rubrospinal tract; $C 2$ \& C5= cervical level 2, 5; Th1 \& Th5= thoracic level 1 \& 5 . 


\subsubsection{Anterograde tracing of RST immediately after dorsal spinal cord hemisection with AAV.EGFP}

In search for a better labeling technique and considering a rapid and significant RN neuronal atrophy following $\mathrm{SCl}$, we investigated the possibility of AAV-mediated labeling immediately following the SCI. In the second set of experiments (Table 8 and Figure 9), the animals were therefore first subjected to a dorsal spinal cord hemisection and this was immediately followed by stereotactic RN injections with AAV.EGFP. After 4 weeks, the
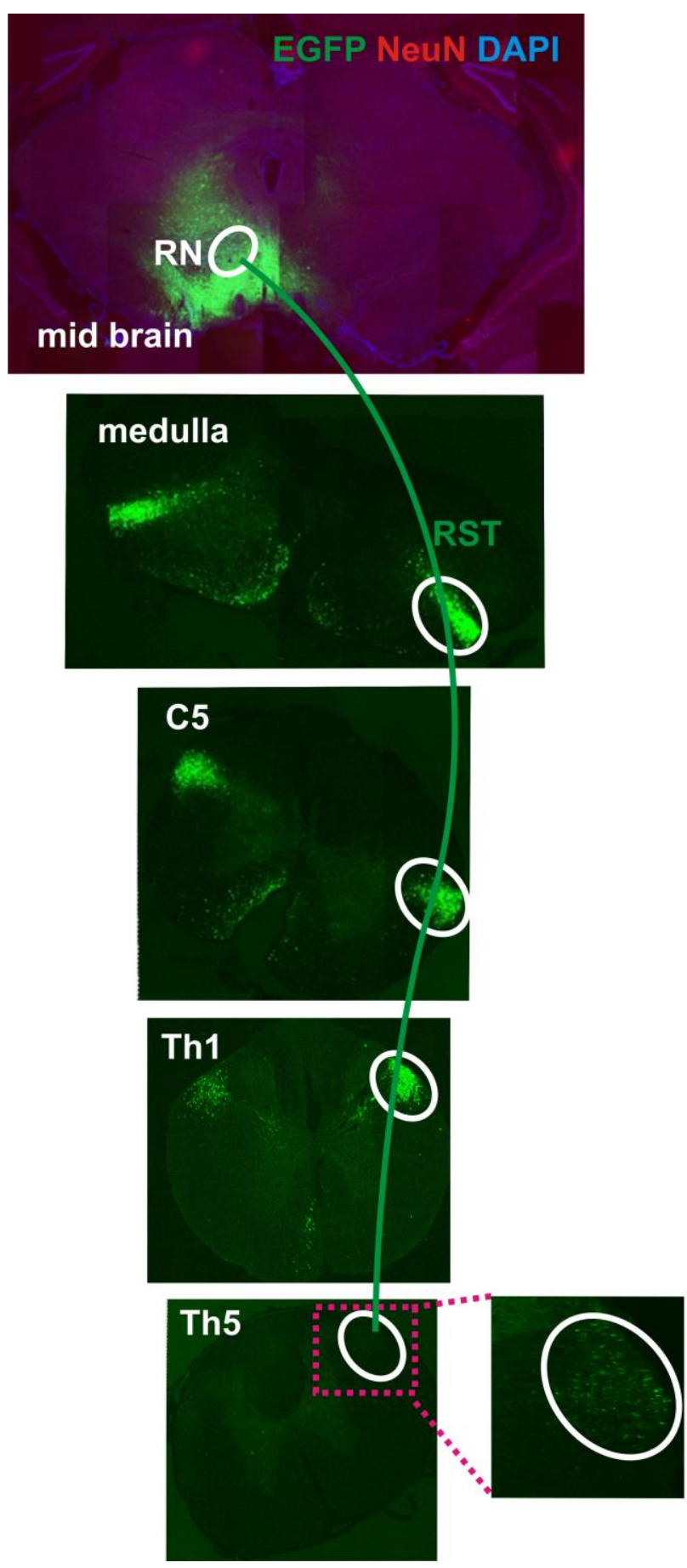
animals were perfused. In this paradigm the AAV vector could successfully label the $\mathrm{RN}$ and subsequently trace the RST both at cervical and thoracic levels until the lesion at Th8 (Figure 23).

Figure 23. Anterograde tracing of RST immediately after dorsal spinal cord hemisection. Representative coronal midbrain section showing $R N$ injection with AAV-mediated EGFP-expression (green), immunolabeling with NeuN (red). The green line depicts the path of the RST (white ovals) labeled with EGFP at the level of the medulla, C5, Th1 and Th5 proximal to the lesion. The dotted pink square at Th5 is shown at a higher magnification. $R N=$ red nucleus; $\quad R S T=$ rubrospinal tract; C5= cervical level 5; Th1 \& Th5 $=$ thoracic level $1 \& 5$. 
These anterograde tracing results were now used for the second experimental paradigm (Figure 9a), where the animals were subjected to dorsal spinal cord hemisection followed by immediate RN AAV-injections. It has to be noted, however, that in this paradigm AAV-vector-mediated tracing of RST took place at a time, where axonal degeneration was just initiated by SCl. However, this paradigm allowed us to clearly identify regenerative changes following lesion, because all axons visualized in this paradigm have obtained their label after spinal cord hemisection.

4.3 Gene therapy with AAV.BAG1, AAV.reggie1, AAV.miRNA-134, and AAV.sh.ROCK2 at 12 weeks after dorsal spinal cord hemisection

4.3.1 Experimental set up for gene therapy with AAV.BAG1, AAV.reggie1, AAV.miRNA-134, and AAV.sh.ROCK2 at 12 weeks after dorsal spinal cord hemisection

To demonstrate sufficient transduction of the RN and the RST after stereotactic injections with AAV vectors and to assure the expected extent of the lesion, preliminary experiments were performed. Animals were subjected to right dorsal spinal cord hemisection at Th8 followed by immediate stereotactic AAV injection in the left RN (Figure 24a). Three weeks after AAV injection the animals were perfused. Co-expression of EGFP in all AAV vectors could visualize that the stereotactic viral injections successfully transduced a defined area of the midbrain including the RN (Figure 24b) and projecting axons in the contralateral RST (Figure 24d). The right dorsal SC hemisection resulted in a complete transection of the RST (Figure 24d). Acute right dorsal hemisection of the RST (3 days after the RST lesion) also resulted complete transection of RST (Figure 24c). We confirmed overexpression of BAG1 and down-regulation of ROCK2 in the RN after viral injections by immunohistochemical analyses of midbrain sections (Figure 25). 
rostral
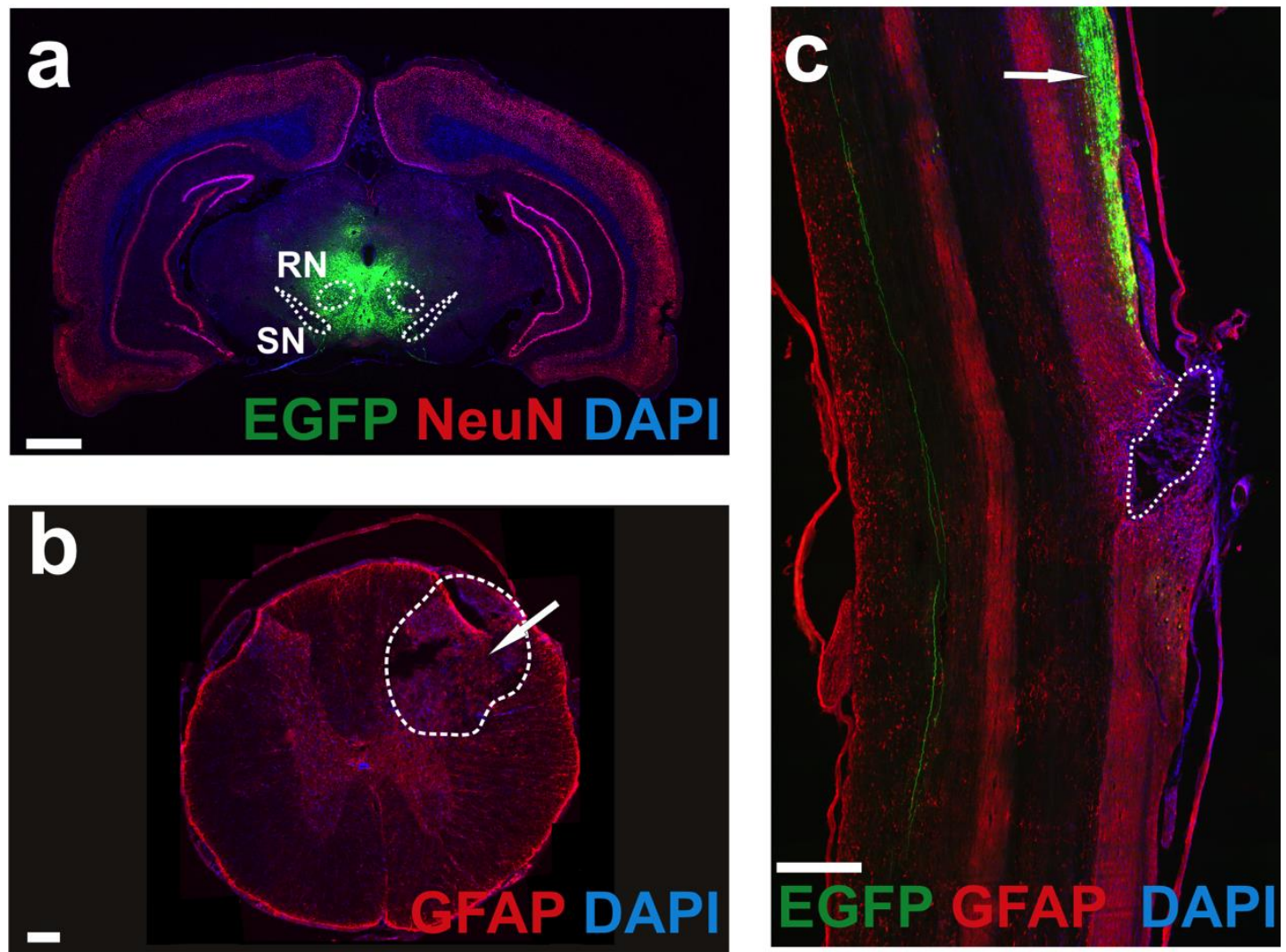

\section{caudal}

Figure 24. Experimental paradigm for AAV.BAG1 and AAV.sh.ROCK2 gene therapy. (a) Representative coronal brain section showing $A A V$ mediated EGFP-expression (green) at 3 weeks after AAV injection to the ipsilateral RN. Immunolabeling for NeuN (red), DAPI (blue). Scale bar 1000 um. (b) Representative transverse spinal cord section showing the dorsal hemisection at three days after lesion. Immunolabeling for GFAP (red), DAPI (blue). White dashed lines mark the lesioned area after hemisection, arrow points to the rubrospinal tract. Scale bar $200 \mu \mathrm{m}$. (c) Representative longitudinal section of the spinal cord showing the anterograde labeling of RST by AAV-mediated EGFP-expression (arrow). Immunolabeling for GFAP (red), DAPI (blue). White dashed lines mark the lesion. Scale bar $500 \mu \mathrm{m}$. $A A V=a d e n o-a s s o c i a t e d$ virus; $R N=$ red nucleus; $R S T=$ rubrospinal tract; $S C I=$ spinal cord injury; $S N=$ substantia nigra. 
a

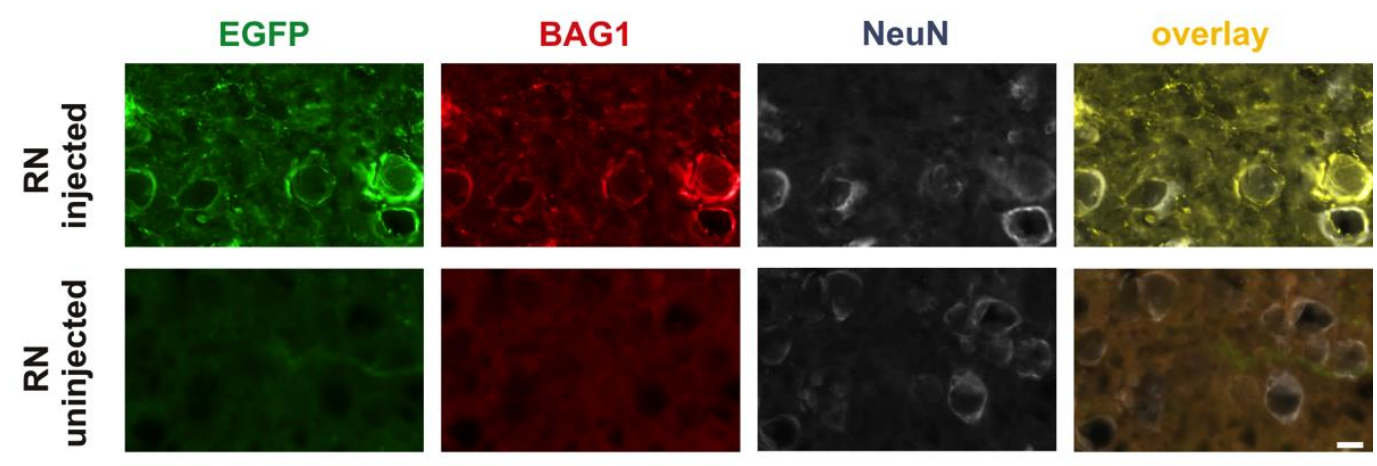

b
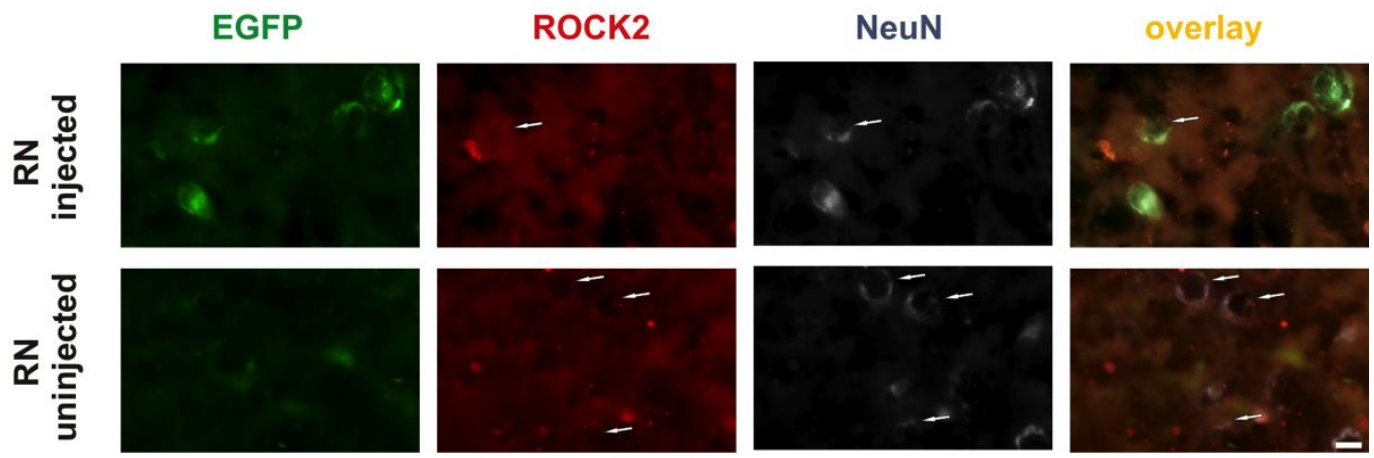

Figure 25. Regulation of BAG1 and ROCK2 expression in the $R N$ by $A A V$ vectors. (a) Representative photomicrographs showing AAV.BAG1transduced RN neurons (EGFP; green), immunolabeling for BAG1 (Cy3; red) and NeuN (Cy5; white). The injected (upper panel) and uninjected (lower panel) sides of the midbrain are shown 12 weeks after dorsal hemisection. (b) Representative photomicrographs showing AAV.sh.ROCK2-transduced $R N$ neurons (EGFP; green), immunolabeling for ROCK2 (Cy3; red) and NeuN (Cy5; white). The injected (upper panel) and uninjected (lower panel) sides of the midbrain are shown 12 weeks after dorsal hemisection. The white arrows point to ROCK2 expressing neurons (cytoplasmic red puncta). Scale bars: $10 \mu \mathrm{m}$.

\subsubsection{Effect of AAV.reggie1 and AAV.miRNA-134 on the red nucleus} after dorsal spinal cord hemisection

In contrast to animals injected with AAV.BAG1, AAV.EGFP, AAV.sh.ROCK2 or AAV.sh.firefly the expression levels of EGFP were found to be very weak for animals injected with AAV.reggie1. In addition, staining with NeuN suggested a toxic effect of AAV.reggie1 expression as the number of surviving RN neurons was reduced (Figure 26d).

Similarly, EGFP expression levels in the RN in AAV.miRNA-134-injected animals were very low at 12 weeks after spinal cord hemisection. The NeuN 
immunostaining also indicated loss of neuronal numbers by 12 weeks after the lesion, suggesting toxic effects of the vector (Figure 26c).

a

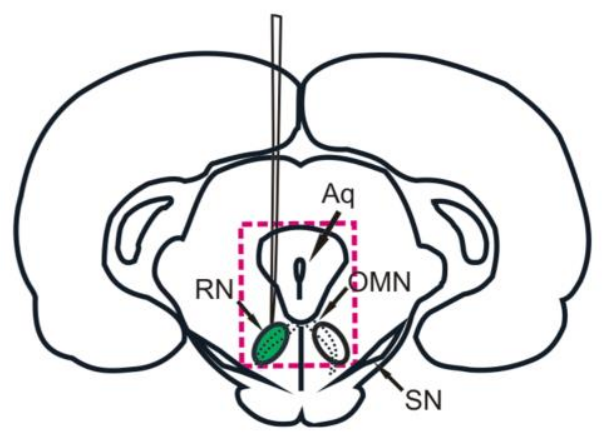

b AAV.miRNA-134
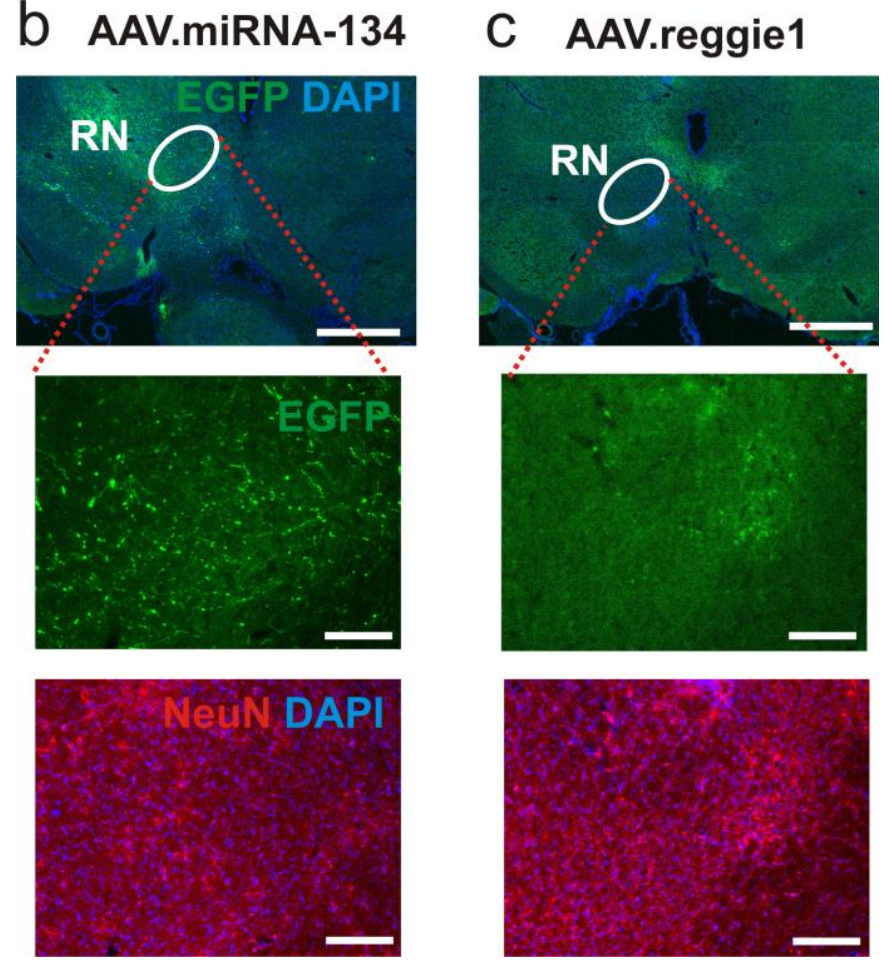

Figure 26. RN transduction by AAV.miRNA-134 and AAV.reggie1. (a) Scheme of a coronal midbrain section showing an overview of the areas depicted in $\boldsymbol{b}$ and $\boldsymbol{c}$. The needle tract for stereotactic AAV injections is marked. The area surrounded by the dashed line red box is shown in the RN overview in $\boldsymbol{b}$ and $\boldsymbol{c}$. Red nucleus (RN), aqueduct $(A q)$, nucleus of oculomotor nerve (OMN), substantia nigra (SN). Scale bars $1000 \mu m(b, c)$ Representative photomicrographs of the midbrain (RN overview) of animals injected with AAV.miRNA-134 (b) and AAV.reggie1 (c). Higher magnification images for the RN labeled by EGFP (green), NeuN (red) and DAPI (blue) at 12 weeks after dorsal spinal hemisection. Scale bars $1000 \mu m$ (RN overview), $500 \mu m$ (higher magnification of $R N$ ).

Due to these obvious toxic effects in the $\mathrm{RN}$ by both AAV.reggie1 and AAV.miRNA-134 vectors, these were excluded from further analysis. 


\subsubsection{AAV.BAG1 and AAV.sh.ROCK2 improve survival of RN neurons 12 weeks after dorsal spinal cord hemisection}

It has been shown previously that $\mathrm{RN}$ neurons degenerate or undergo severe atrophy following RST lesion and this could represent a handicap for regeneration (Kwon et al., 2002a; Tseng et al., 1996). In order to evaluate the neuroprotective effects of AAV.BAG1 and AAV.sh.ROCK2 on RN neurons 12 weeks after RST lesion, midbrain sections at the level of the RN were immunolabeled with antibodies against NeuN. NeuN quantifications were performed both in the injected RN (corresponding to the lesioned RST) and un-injected (corresponding to the unlesioned RST) which served as an internal control for each animal. In all experimental approaches, we compared each AAV vector with its appropriate control only, e.g. AAV.BAG1 vs. AAV.EGFP (both vectors overexpressing a protein of interest) and AAV.sh.ROCK2 vs. AAV.sh.firefly (both vectors expressing a functional shRNA). The number of NeuN-positive cells in the RN was significantly increased in the groups transduced with AAV.BAG1 compared to AAV.EGFP (173.0 \pm 7.0 vs. $80.7 \pm 11.0$ NeuN-positive cells per section). Animals injected with AAV.sh.ROCK2 also showed significantly higher numbers of NeuN-positive neurons in the $\mathrm{RN}$ as compared to AAV.sh.firefly-treated controls (85.5 \pm 6.3 vs. $51.2 \pm 3.2$ NeuN-positive cells per section). The numbers of NeuN-positive cells in the contralateral uninjected RN did not differ among the groups (Figure 27b \& c).

As severe atrophy was reported for the RN neurons after the RST lesion (Ruitenberg et al., 2004),(Kwon et al., 2004), we performed a quantification of RN neuron soma size. Most of the neuronal cell bodies in the AAV.EGFP injected animals had sizes of less than $300 \mu \mathrm{m}^{2}$ and there were none, which were larger than $600 \mu^{2}$. In contrast AAV.BAG1-injected animals showed a larger spectrum of soma sizes with more than $20 \%$ of the RN cell bodies being larger than $600 \mu^{2}$. Differences were less pronounced among the AAV.shRNA-injected animals. However, AAV.sh.ROCK2-injected animals showed more larger-sized cell bodies than AAV.shRNA-firefly-injected controls (Figure $\mathbf{2 7 d}$ ). The somata of the $\mathrm{RN}$ neurons of the uninjected side 
were larger compared to the injected $\mathrm{RN}$ side for AAV.EGFP, AAV.sh.ROCK2 and AAV.sh.firefly groups while soma sizes were similar to the uninjected side in the AAV.BAG1-treated group (Figure 27b).

Overall, this data demonstrates that AAV.BAG1 improved neuronal survival and reversed the lesion-induced atrophy of the RN neurons after RST lesion. AAV.sh.ROCK2 had also improved the survival of RN neurons and partially improved the atrophy of $\mathrm{RN}$ neurons compared to its control. In general, neuronal numbers in AAV.shRNA-injected animals were lower than in animals injected with AAV over expressing a protein. This is likely to be attributed to vector-inherent toxicity due to chronic shRNA-expression and has been previously reported (Ehlert et al., 2010). 
a

b
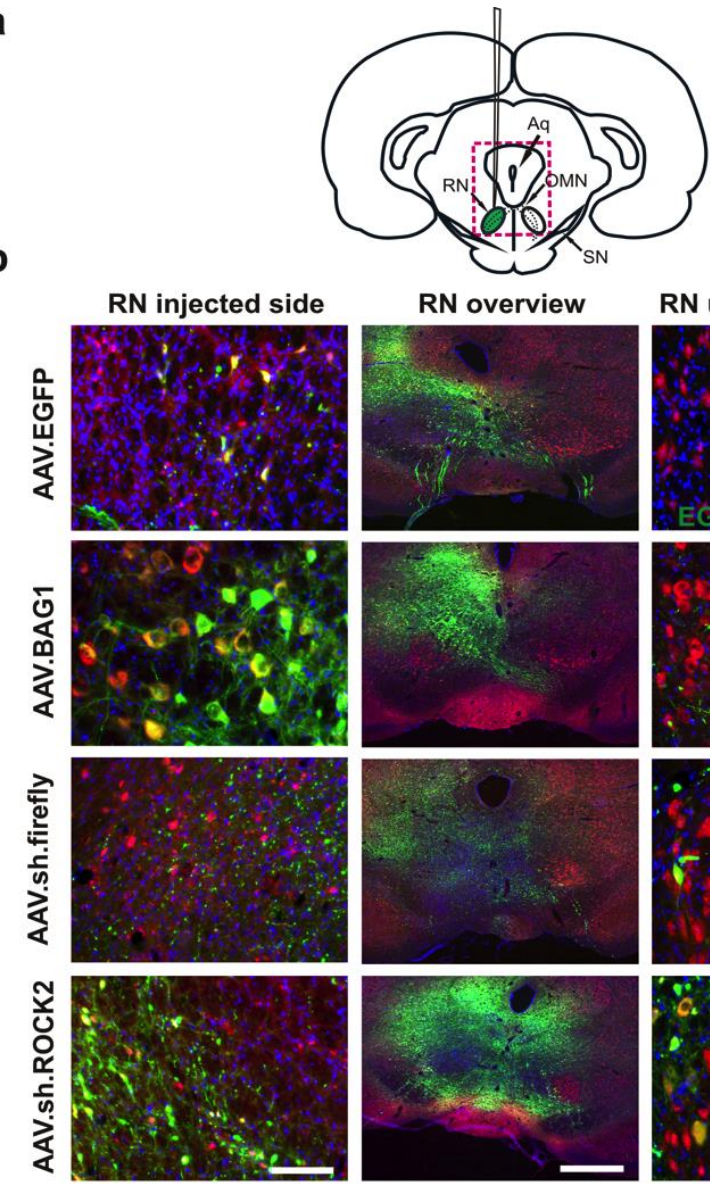

c

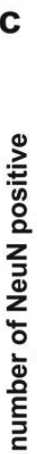

d
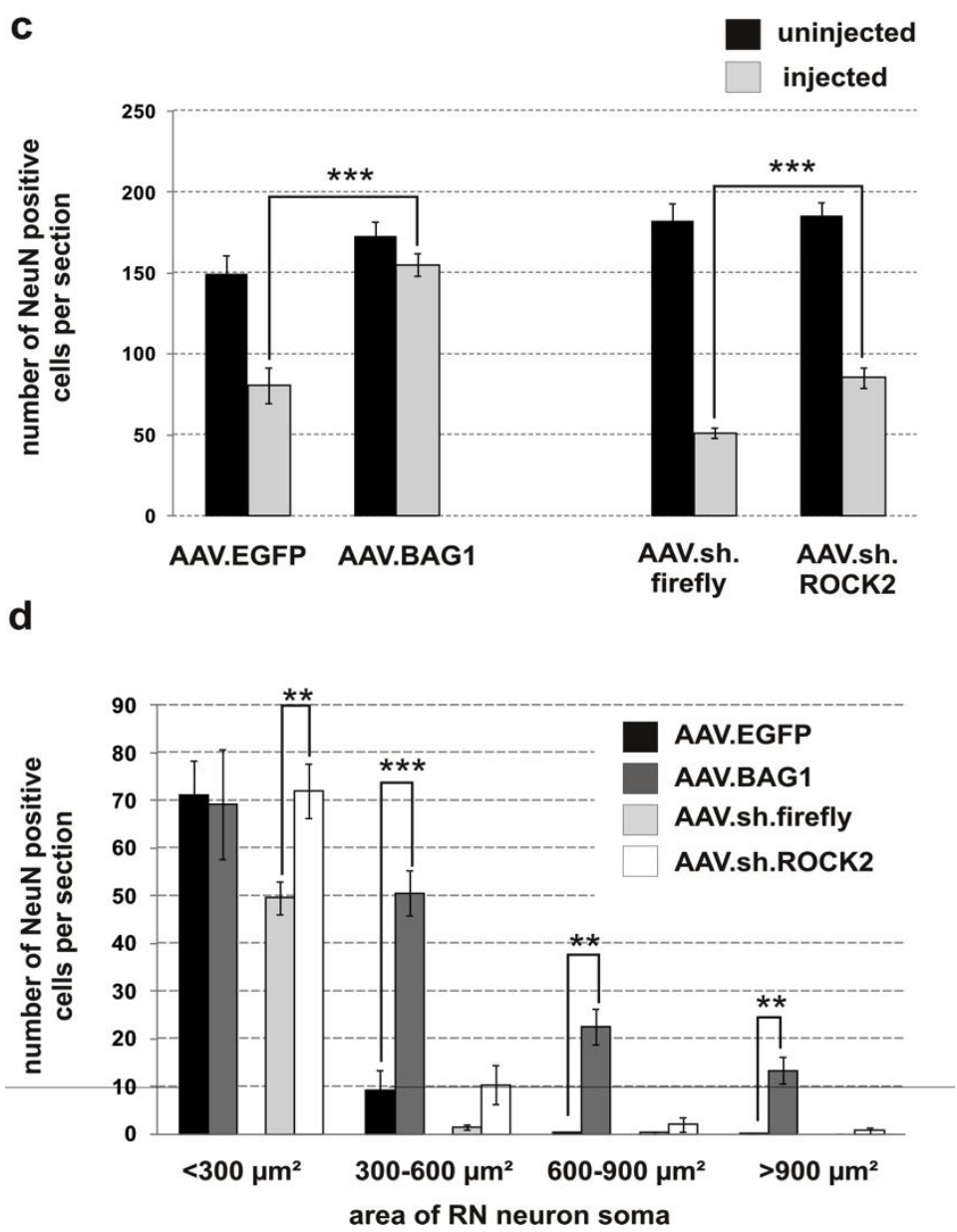

Figure 27. Neuronal survival in the $R N$ after AAV.BAG1 and AAV.sh.ROCK2 gene therapy 12 weeks after lesion. (a) Scheme of a coronal brain section showing an overview of the areas depicted in $\boldsymbol{b}$ : red nucleus (RN), aqueduct $(A q)$, nucleus of oculomotor nerve $(O M N)$, substantia nigra (SN). The needle tract for stereotactic AAV injections is marked. The area surrounded by the dashed line red box is shown in the $R N$ overview in b. (b) Representative

photomicrographs of the midbrain (RN overview, middle panel) and of both injected (left panel) and uninjected $R N$ (right panel) in higher magnification for all $A A V$ vectors used in this study. AAV-mediated EGFP-expression

(green) delineates the transduced area. Immunolabeling for NeuN (red), DAPI (blue). Scale bars $1000 \mu \mathrm{m}$ (RN overview), $500 \mu \mathrm{m}$ (injected $R N$, uninjected $R N)$. (c) Quantification of NeuN-positive cells in the injected (lesioned RST) and uninjected (unlesioned RST) RN. (d) Quantification of the area of the neuronal somata in the $R N$ of the AAV-injected side. Bars in $\boldsymbol{c}$ and $\boldsymbol{d}$ represent means \pm SEM. Differences were considered significant with * $p<0.05$; ${ }^{* *} p<0.01$; ${ }^{* * *} p<0.001$. AAV.EGFP, $n=4 ; \quad$ AAV.BAG1, $n=8$, AAV.sh.firefly, $\quad n=7$; 


\subsubsection{Axonal sprouting at cervical $S C$ is enhanced by treatment with AAV. BAG1 and AAV.sh.ROCK2}

To investigate the effects of AAV.BAG1 and AAV.sh.ROCK2 gene therapies on collateral sprouting, proximal to the lesion at the cervical level, animals were perfused 12 weeks after dorsal spinal cord hemisection and AAV injection. A total of 5 transverse cryosections of the cervical SC were used at the level of C2-C6. In order to assess the distribution of RST sprouts towards the Rexed's laminae $\mathrm{V}$ and $\mathrm{VI}$ and towards the dorsal part of lamina VII (Küchler et al., 2002) after the lesion, the number of EGFP-labeled axonal sprouts were counted at defined distances from RST to the central canal (Figure. 28a \& 28b). The number of sprouts was normalized to EGFPtransduction to avoid bias by the individual variability of transduction efficiency between different animals. A significant increase in axonal sprouting (up to 2-fold) was observed with AAV.BAG1 vs. AAV.EGFP and this effect was especially pronounced at distances of 800-1200 $\mu \mathrm{m}$ from the RST. The effect of AAV.sh.ROCK2 appeared even more pronounced, since AAV.sh.firefly control-injected animals showed a minor sprouting response (Figure 29 b \& 29c). We observed that the extent of sprouting was dependent on the spinal cord levels and mostly pronounced in cranially investigated level $\mathrm{C} 2$. Axonal sprouting decreased towards more caudally located levels with the lowest values at C6 (Figure 30) and this trend was similarly observed in all treatment groups. In addition to this, we also observed that the axonal sprouts of the RST co-localize with synaptophysin in the close vicinity of interneurons of Rexed's laminae $\mathrm{V}$ and $\mathrm{VI}$ in the cervical SC. This suggests that the establishment of synaptic contacts onto interneuron's by the RST sprouts and the density of such synaptic contacts was highest in AAV.BAG1- and AAV.sh.ROCK2-treated groups (Figure 31). 

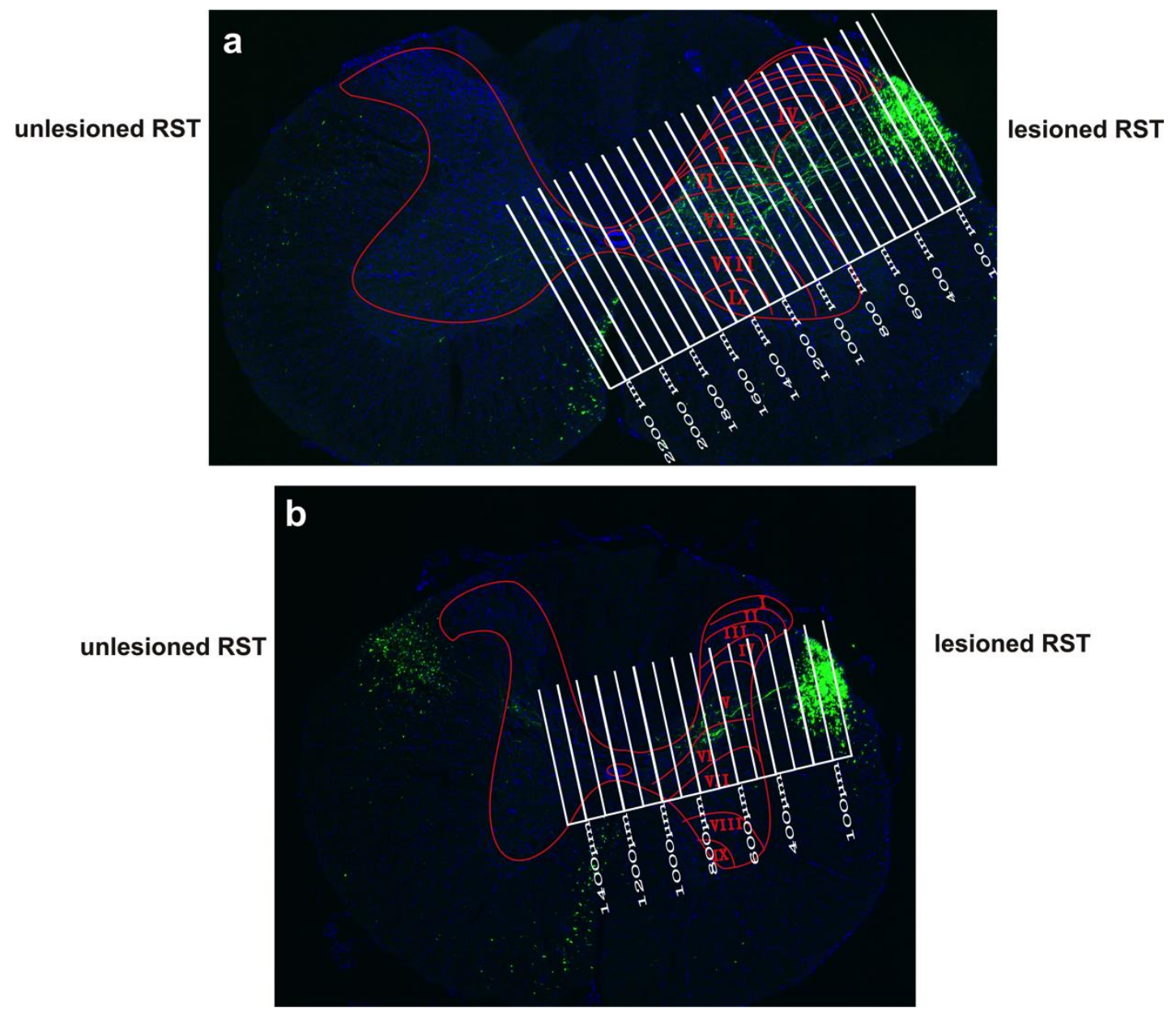

Figure 28. Model of axonal sprouting quantification at the cervical and thoracic levels. Scheme of axonal sprouting quantification of the transverse cervical (a) and thoracic (b) spinal cord depicting the location of RST axons (green), Rexed's laminae of the spinal cord gray matter (pink lines) and the scale (white lines) superimposed for quantification. 
Figure 29. Axonal sprouting in the cervical SC after AAV.BAG1 and AAV.sh.ROCK2 gene therapy at 12 weeks after lesion.

(a) Scheme of a transverse cervical SC section depicting the location of the micrographs

(squares) shown in b. Rexed's laminae (I-IX) of the spinal cord gray matter are marked for orientation.

(b) Representative photomicrographs of transverse cervical spinal cord sections of animals, which have been injected in the $R N$ with AAV.EGFP, AAV.BAG1, $A A V$.sh.firefly and AAV.sh.ROCK2.

Axonal sprouting to the gray matter is

a

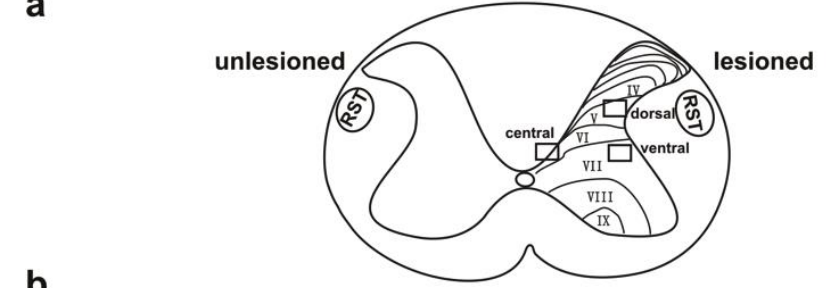

\section{overview}

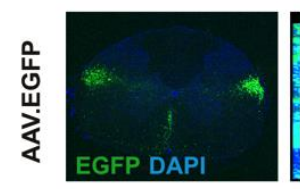

dorsal

ventral
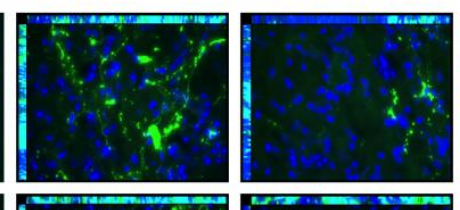

central
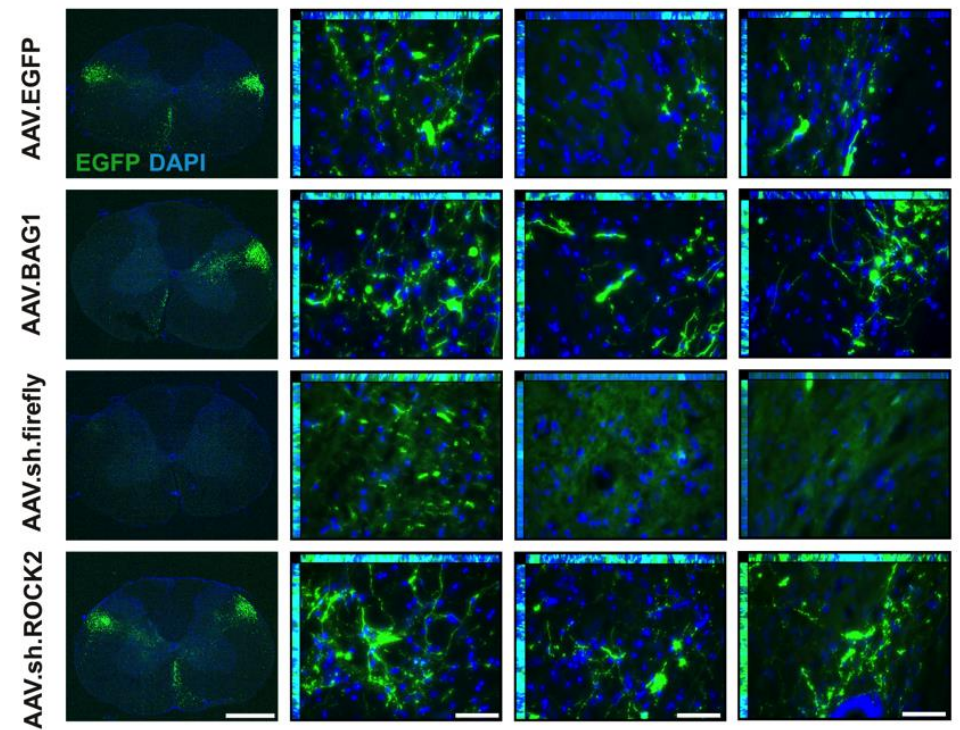

c

AAV.EGFP AAV.BAG1 AAV.sh.firefly AAV.sh.ROCK2

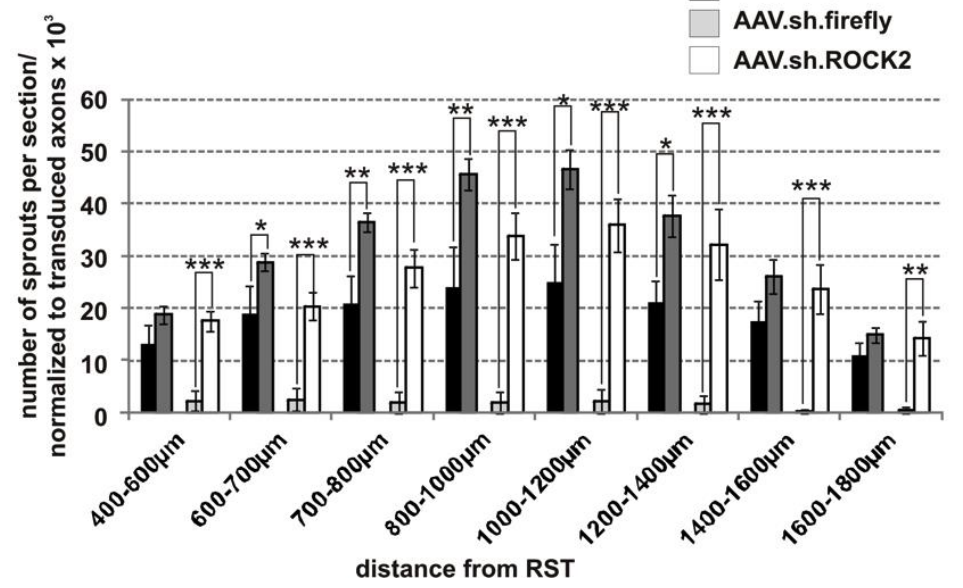

visualized by AAV-mediated EGFP

expression (green), DAPI (blue). Overview and higher magnifications of dorsal, ventral and central areas (see a) are shown. Scale bars: $1000 \mu \mathrm{m}$ (overview), $50 \mu \mathrm{m}$ (dorsal, ventral and central). (c) Quantification of axonal sprouting in the cervical spinal cord (C2-C6) at different distances from the RST normalized to RST transduction efficiency (EGFP-positive axons counted at C2). Bars represent the means \pm SEM. Differences were considered significant with ${ }^{*} p<0.05 ;{ }^{* *} p<0.01 ;{ }^{* *} p<0.001$. AAV.EGFP, $n=4$; AAV.BAG1, $n=8$, AAV.sh.firefly, $n=7$, AAV.sh.ROCK2, $n=8$. 
a

b

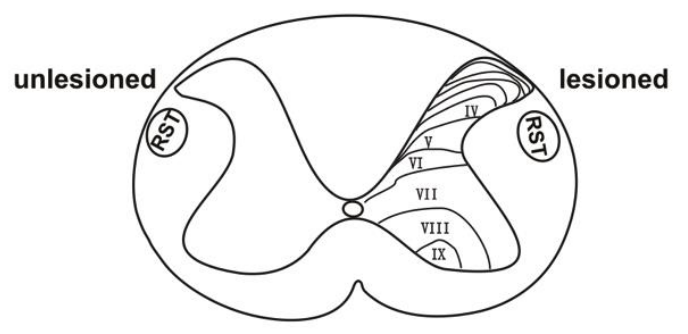

C2

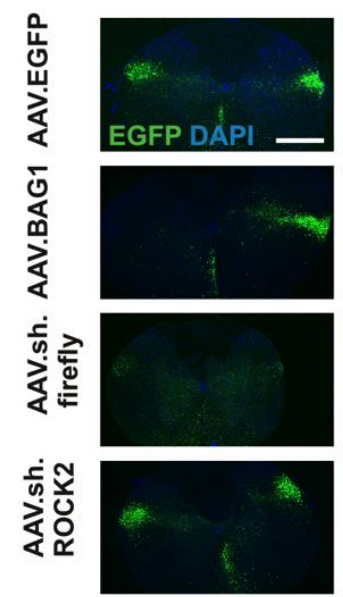

C3
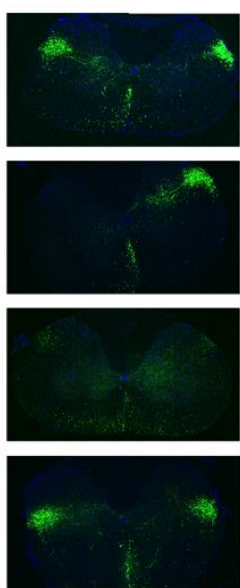

C4
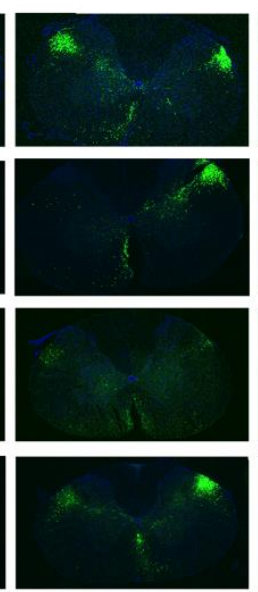

C5
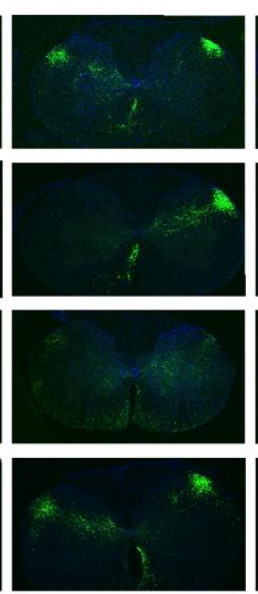

C6

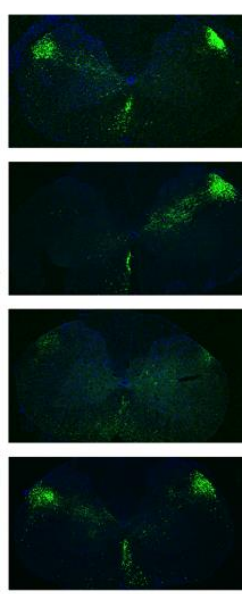

C

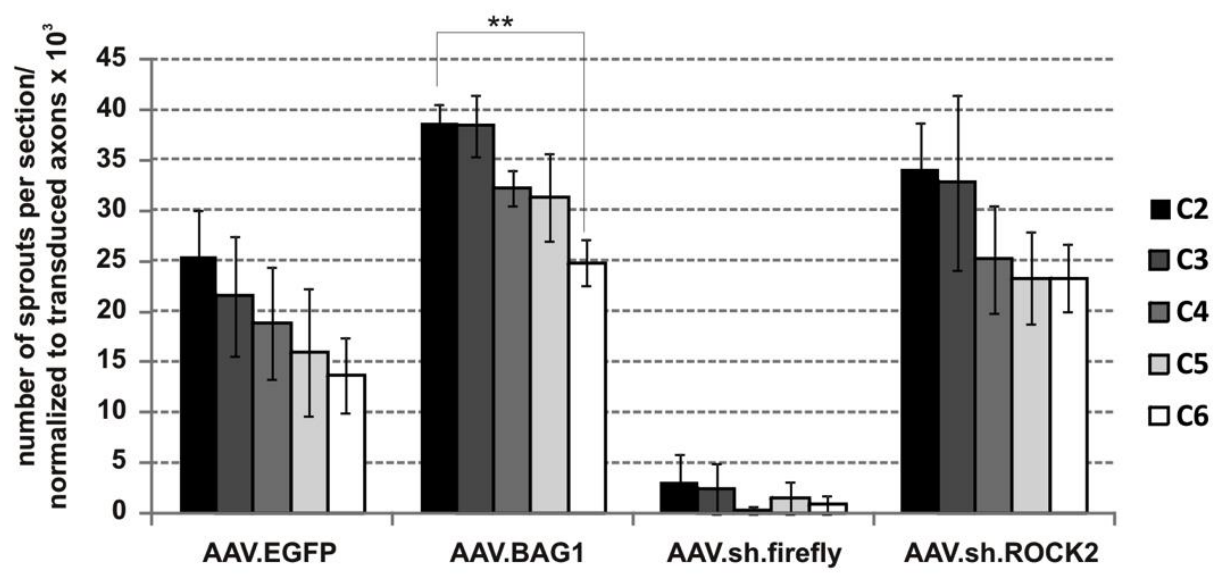

Figure 30. Effect of AAV.BAG1 and AAV.sh.ROCK2 gene therapy on Axonal sprouting at the cervical levels 2 to 6 at 12 weeks after lesion. (a) Scheme of the transverse cervical spinal cord depicting the location of RST and Rexed's laminae (I-IX) of the spinal cord gray matter marked for the orientation. (b) Representative photomicrographs of transverse cervical spinal cord sections $C 2$ to $C 6$ of animals, which have been injected in the $R N$ with AAV.EGFP, AAV.BAG1, AAV.sh.firefly and AAV.sh.ROCK2. Axonal sprouting to the gray matter is visualized by $A A V$-mediated EGFP expression (green), DAPI (blue). Scale bar $1000 \mu \mathrm{m}$. (c) Quantification of differences in axonal sprouting among the cervical spinal cord levels (C2 to C6) normalized to RST transduction efficiency (EGFP positive axons counted at C2) 12 weeks after RST lesion. Bars represent the mean $\pm S E M$. Differences were considered significant with ${ }^{*} p<0.05 ;{ }^{* *} p<0.01 ;{ }^{* *} p<0.001$. AAV.EGFP, $n=4$; AAV.BAG1, $n=8$, AAV.sh.firefly, $n=7 \&$ AAV.sh.ROCK2, $n=8$. 


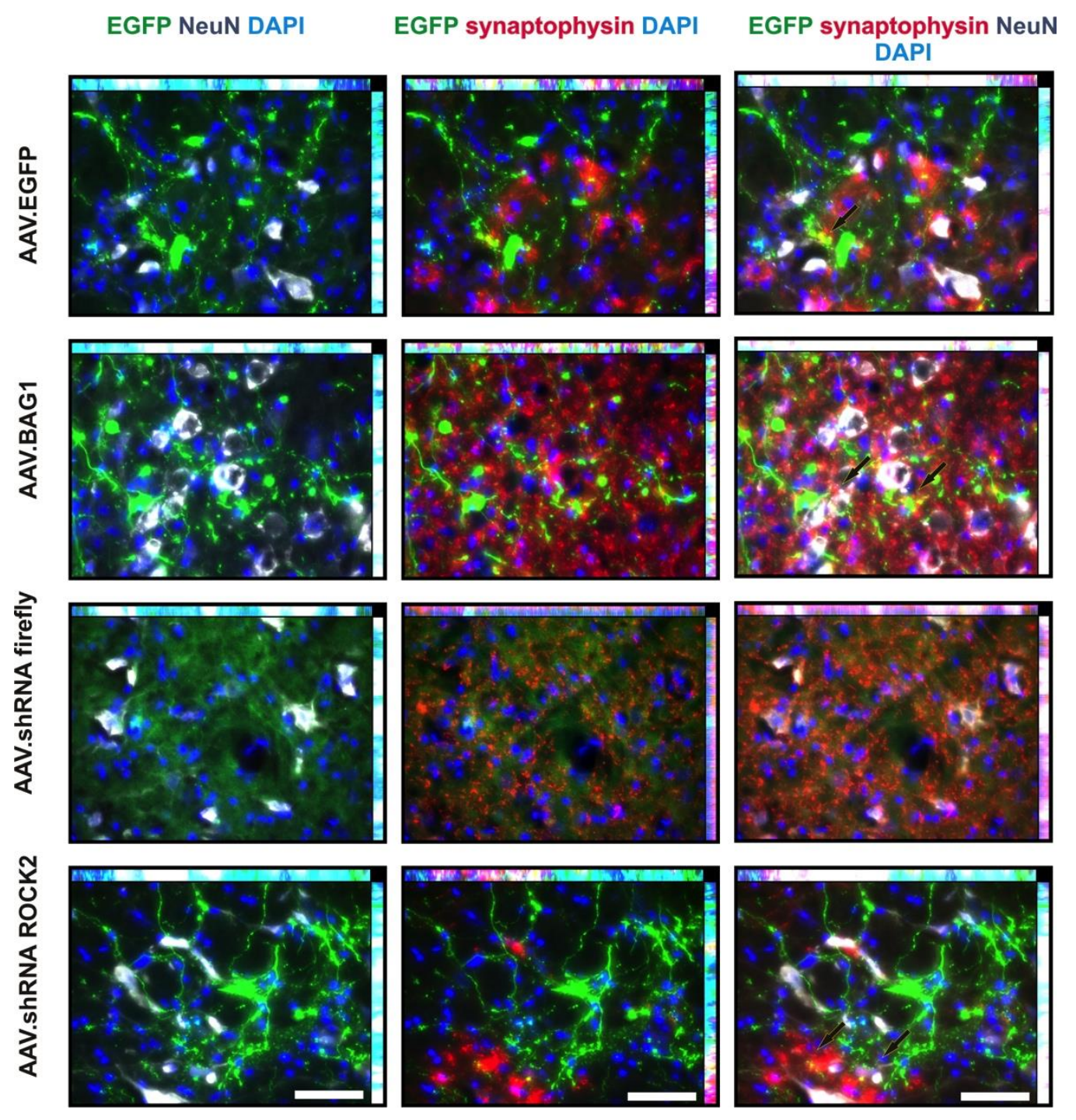

Figure 31. RST sprouts establish contacts with interneurons in the gray matter Rexed's laminae of the cervical spinal cord. Representative photomicrographs of transverse cervical spinal cord sections of animals, which have been injected in the RN with AAV.EGFP, AAV.BAG1, $A A V$.sh.firefly and AAV.sh.ROCK2. Axonal sprouting to the gray matter is visualized by $A A V$-mediated EGFP expression (green), immunolabeling of NeuN (Cy5), synaptophysin (red) and DAPI (blue) and the colocalization of axonal sprouts with synaptophysin and contacts with NeuN (black arrows) in the overlay. Scale bars: $50 \mu \mathrm{m}$. 


\subsubsection{Axonal sprouting at thoracic $S C$ is enhanced by treatment with}

\section{AAV. BAG1 and AAV.sh.ROCK2}

A similar quantification that was obtained at the cervical SC for axonal sprouting was also performed at the thoracic level, i.e. closer to the lesion, but at a larger distance to the affected neuronal somata of the RN. Here, a total of 5 transverse sections of the spinal cord at the levels of Th1-Th5 were examined. Similar to the cervical SC, AAV.BAG1 resulted in stronger axonal sprouting than AVV.EGFP at the thoracic level, although this difference was not significant. In contrast, AAV.sh.ROCK2 significantly improved axonal sprouting as compared to AAV.sh.firefly group. The highest number of sprouting fibers was observed at a distance of $600-800 \mu \mathrm{m}$ from the RST (Figure 32). Consistent to our findings at cervical level, we observed stronger sprouting more at cranial levels than at caudal ones (Figure 33). In general, levels of sprouting at cervical spinal cord were more pronounced than at thoracic levels (Figure 33). Taken together, AAV-mediated overexpression of BAG1 as well as shRNA-mediated downregulation of ROCK2 strongly increased sprouting at cervical and thoracic levels following RST lesion and this sprouting response was inversely correlated with the distance to the neuronal soma. 
Figure 32. Axonal

a sprouting in the thoracic SC after AAV-gene therapy at 12 weeks after lesion. (a) Scheme of a transverse thoracic SC section depicting the location of the micrographs (squares) shown in $\boldsymbol{b}$. Rexed's laminae (I$I X)$ of the spinal cord gray matter are marked orientation.

Representative photomicrographs of transverse thoracic spinal cord sections of animals, which have been injected in the $R N$ with AAV.EGFP, for

(b) b
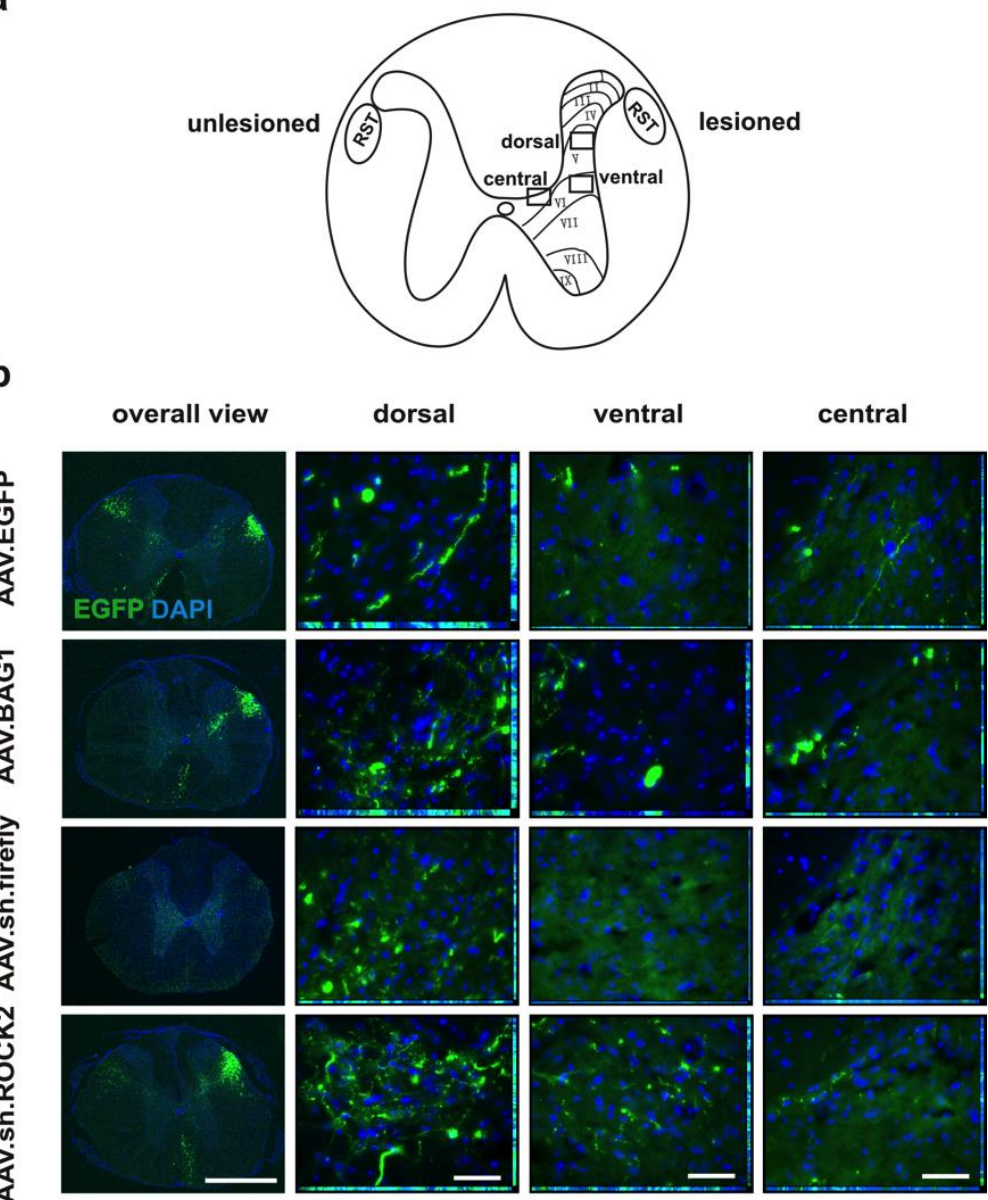
AAV.BAG1, $A A V$.sh.firefly and AAV.sh.ROCK2.

Axonal sprouting to the gray matter is visualized by AAVmediated EGFP expression (green), $D A P I$ (blue).

Overview and higher magnifications of

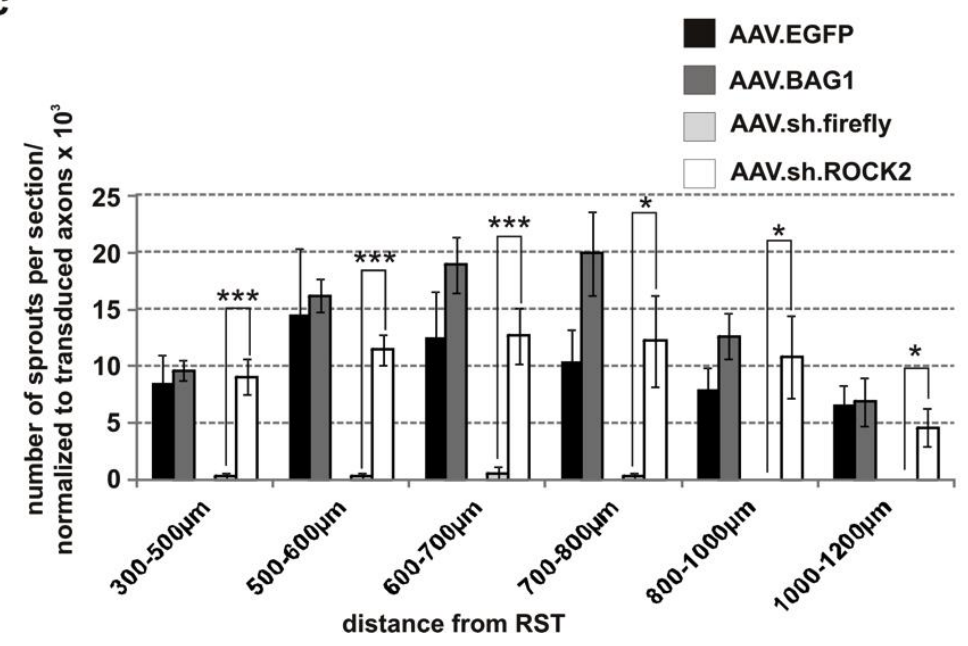
dorsal, ventral and central areas (see a) are shown. Scale bars: $1000 \mu \mathrm{m}$ (overview), $50 \mu \mathrm{m}$ (dorsal, ventral and central). (c) Quantification of axonal sprouting in the thoracic spinal cord (Th1-Th5) at different distances from the RST normalized to RST transduction efficiency (EGFP-positive axons counted at C2). Bars represent the means $\pm S E M$. Differences were considered significant with ${ }^{*} p<0.05 ;{ }^{* *} p<0.001 . \quad$ AAV.EGFP, $n=4$; AAV.BAG1, $n=8$, AAV.sh.firefly, $n=7$, AAV.sh.ROCK2, $n=8$. 
Figure 33. Effect of AAV.BAG1 and AAV.sh.ROCK2 gene therapy on Axonal sprouting at the thoracic levels 1 b to 5 at 12 weeks after lesion. (a) Scheme of the transverse thoracic spinal cord depicting the location of RST and Rexed's laminae (I-IX) of the spinal cord gray matter marked for the orientation.

Representative photomicrographs of transverse thoracic spinal cord sections Th1 to Th5 of animals, which have been injected in the $R N$ with AAV.EGFP, AAV.BAG1, $A A V$.sh.firefly and AAV.sh.ROCK2.

Axonal sprouting to the gray matter is visualized by $A A V$ mediated EGFP expression (green), DAPI (blue). Scale bar $1000 \mu m$. (c) Quantification

differences in axonal sprouting among the thoracic spinal cord levels (Th1 to Th5) normalized to RST transduction efficiency (EGFP positive axons counted at C2) 12 weeks after RST lesion. (d) Axonal sprouting quantification comparison between cervical and thoracic spinal cord 12 weeks after RST lesion. Bars represent the mean $\pm S E M$. Differences were a
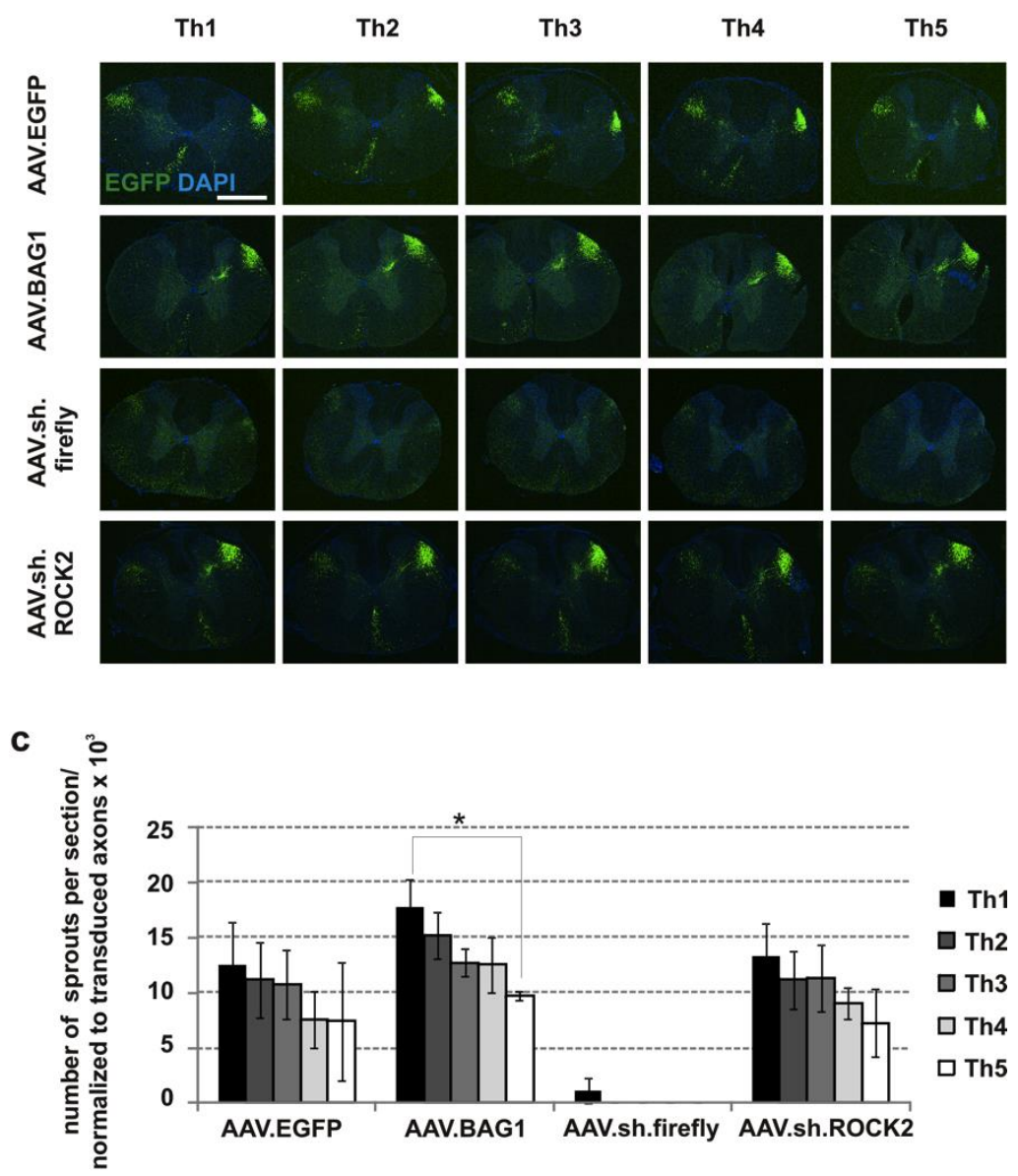

d

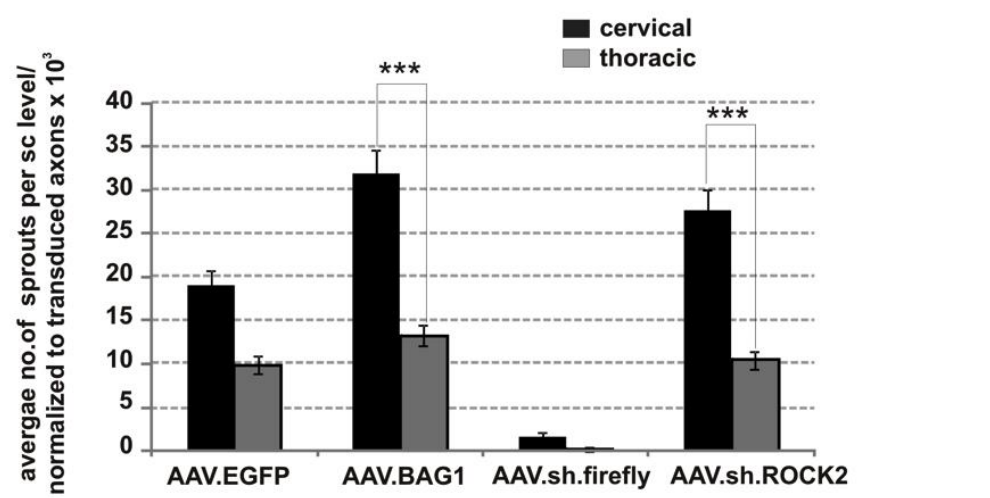


considered significant with ${ }^{*} p<0.05 ;{ }^{* *} p<0.01 ;{ }^{* * *} p<0.001$. AAV.EGFP, $n=4$; AAV.BAG1, $n=8$, AAV.sh.firefly, $n=7 \& A A V$.sh.ROCK2, $n=8$.

\subsubsection{Effects of AAV.BAG1 and AAV.sh.ROCK2 on lesion size and axonal degeneration}

Since lesion size has a strong influence on functional outcome, we quantified the lesion size to exclude any effects of extent of the lesion on AAV gene therapy outcome. For this, horizontal sections of the spinal cord at RST level were immune-labeled with antibodies against GFAP and the lesion area was defined in the absence of GFAP immunostaining. However, no significant differences were observed between treatment groups in regard to the lesion size (Figure 34r). On the other hand stabilization of lesioned axons has been shown to be beneficial for axonal regeneration (Hellal et al., 2011). We thus assessed the impact of gene therapy on lesion-induced axonal degeneration by quantification of the EGFP-labeled RST axons at different distances from the proximal lesion border. Animals treated with AAV.BAG1 showed more EGFP-positive axons at all distances from the lesion than AAV.EGFP-treated animals, which was significant at $100-200 \mu \mathrm{m}$ (Figure 34a-d, e-h \& q). More axons were stabilized in the AAV.sh.ROCK2-treated group compared to the control AAV.sh.firefly group. However, this observation was not statistically significant (Figure 34 i-I, $\mathbf{m}$ \& q). No EGFP-positive fibers passing through or beyond the lesion were observed in any of the treatment groups. 


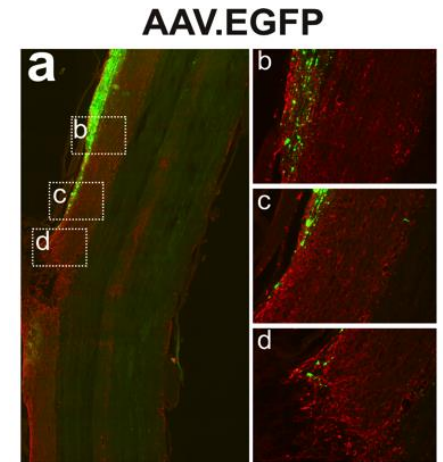

AAV.sh.firefly
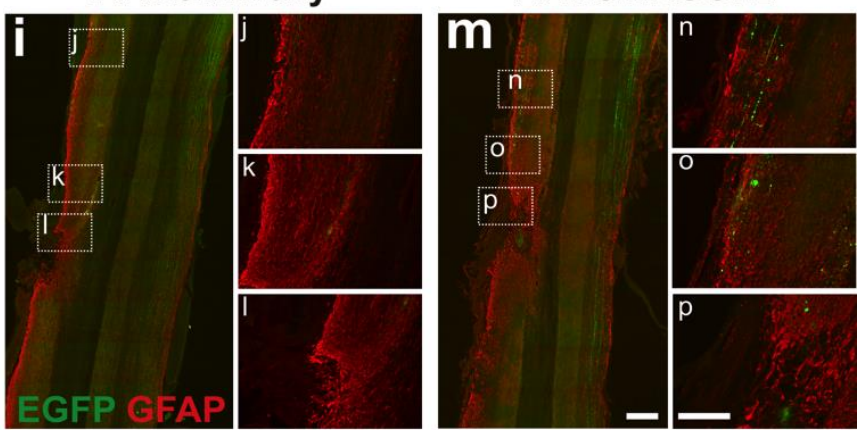

$\mathbf{q}$
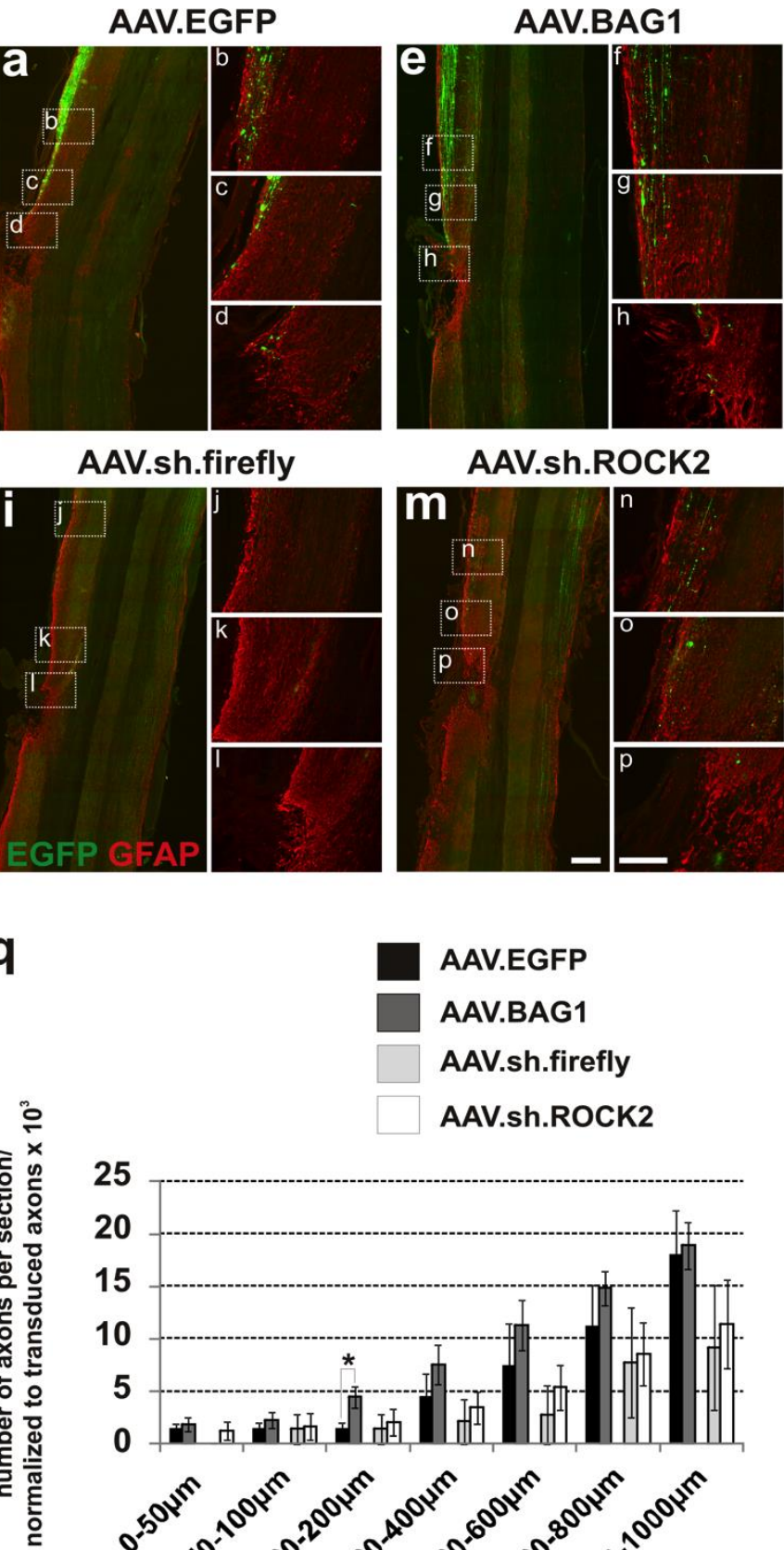

Figure 34. Effects of AAV.BAG1 and AAV.sh.ROCK2 gene therapy on lesion size and axonal degeneration at 12 weeks after dorsal SC hemisection.

Representative

photomicrographs of longitudinal SC sections of animals injected with AAV.EGFP (a-d), AAV.BAG1 (e-h), AAV.sh.firefly (i-I) and AAV.sh.ROCK2 (m-p) into the RN. Overviews (a, e, $\boldsymbol{i}$, m) and higher magnification insets $(\boldsymbol{b}-\boldsymbol{d}$, $\boldsymbol{f}$ $\boldsymbol{h}, \boldsymbol{j}-\boldsymbol{l}, \boldsymbol{n}-\boldsymbol{p})$ show $A A V$ mediated expression of EGFP in the rubrospinal axons proximal to the lesion. Immunolabeling for GFAP (red). Scale bars $500 \mu m(a, e, i, m)$ and 100 $\mu m$ (b-d, $\boldsymbol{f}-\boldsymbol{h}, \boldsymbol{j}-\boldsymbol{l}, \boldsymbol{n}-\boldsymbol{p}) . \quad(\boldsymbol{q})$ Quantification of RST axon degeneration. Axonal profiles counted a different distances proximal to the lesion and normalized to transduction (EGFP positive axons counted at C2). (r) Lesion size quantification. The GFAPnegative area was taken as lesion area. Bars represent the means $\pm S E M$. Differences were considered significant with ${ }^{*} p<0.05$. AAV.EGFP, $n=4$; AAV.BAG1, $n=8$, AAV.sh.firefly, $\quad n=7 \quad$ \& AAV.sh.ROCK2, $n=8$. 


\subsubsection{Effect of AAV.BAG1 and AAV.sh.ROCK2 on motor recovery}

Quantification of neuronal survival, axonal degeneration, sprouting and lesion size precisely characterize effects of $A A V$ gene therapy on a histological level and may suggest subclinical effects by AAV-mediated gene therapy. Functional outcome, however, is of strong clinical relevance and therefore motor function was assessed with the BBB open field test and the CatWalk ${ }^{\mathrm{TM}}$ analysis at week 1, 3, 7 and 11 following the lesion (Basso et al., 1995). All the animals scored a complete score (21 points) in the open field test before the lesion. However, one week after the lesion, the score was reduced to 13 \pm 1 in all groups and over the course of 11 weeks animals showed an improved motor function with an increased score of $15 \pm 1$. AAV.BAG1treated animals showed a significant improvement at week 11 compared to week 1, while this was not observed for groups treated with AAV.EGFP, AAV.sh.firefly or AAV.sh.ROCK2. There was no significant difference in the direct comparison of treatment groups and their appropriate controls (AAV.BAG1 vs. AAV.EGFP and AAV.sh.firefly vs. AAV.sh.ROCK2) (Figure 35a). Since this model is a partial lesion model additional and more subtle motor parameters such as paw placement, toe clearance and trunk stability were assessed as a sub score to identify smaller differences. At week one after the lesion all groups showed a marked reduction in the score with no significant differences between AAV.BAG1 and AAV.EGFP, while the score for AAV.sh.ROCK2 was significantly higher than for AAV.sh.firefly. However, only the AAV.BAG1-treated group showed a significantly increased score as compared to AAV.EGFP at the final quantification at week 11 ( $4.5 \pm 0.32$ vs. $3 \pm 0.70$ points). Animals treated with AAV.sh.ROCK2 showed a trend towards a higher score in comparison to their appropriate control AAV.sh.firefly (Figure 35b). A more detailed gait analysis was also performed using the CatWalk ${ }^{\mathrm{TM}}$ system, which showed significant differences between AAV.sh.firefly and AAV.sh.ROCK2 groups at week 1, however there were no significant differences between the treatment groups at later time points (Figure 36). 
a

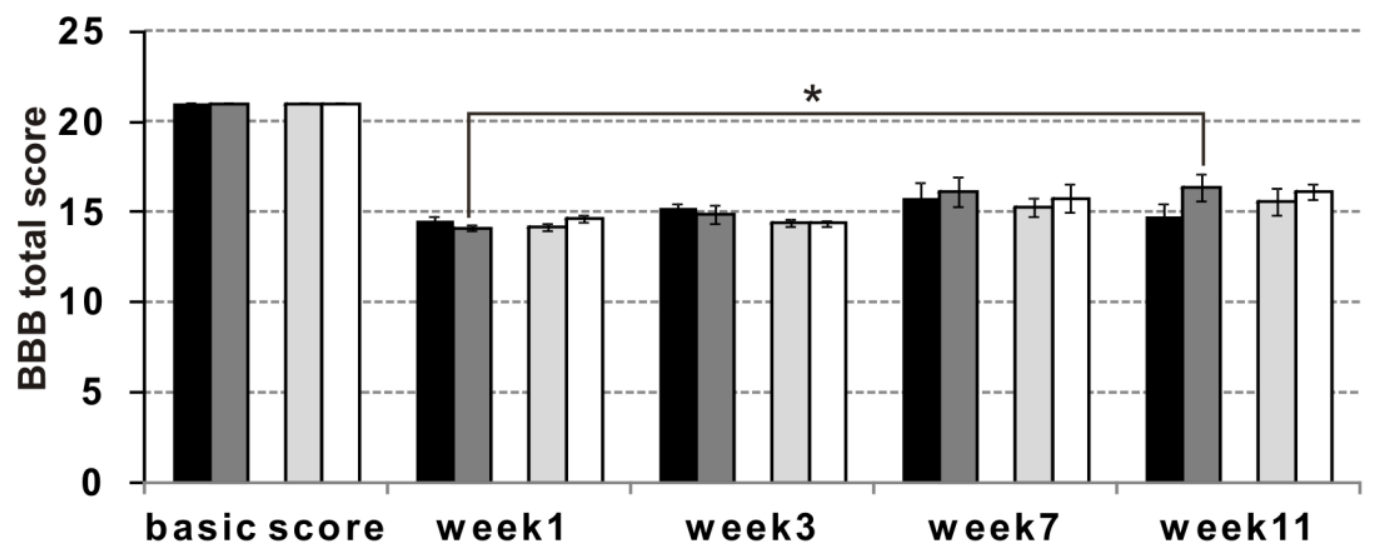

b

AAV.EGFP

AAV.BAG1

AAV.sh.firefly

AAV.sh.ROCK2

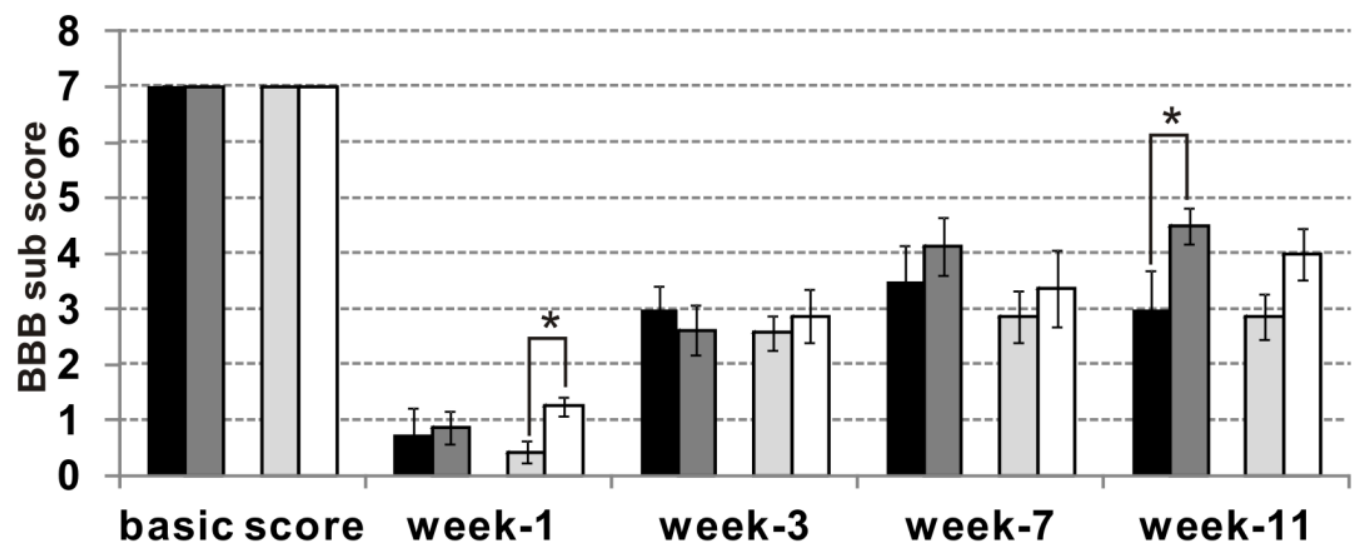

Figure 35. Effect of AAV.BAG1 and AAV.shROCK2 gene therapy on motor behavior quantified by BBB. (a) BBB total score (21 point scale) of the right hind limbmeasured before the lesion and 1, 3, 7 \& 11 weeks after RST lesion. (b) BBB sub score quantification of the right hind limb, considering the parameters toe clearance, predominant paw position and trunk instability. Bars represent the means \pm SEM. Differences were considered significant with ${ }^{*} p<0.05$. AAV.EGFP, $n=4 ; A A V$.BAG1, $n=8$, AAV.sh.firefly, $n=7$ \& AAV.sh.ROCK2, $n=8$. 


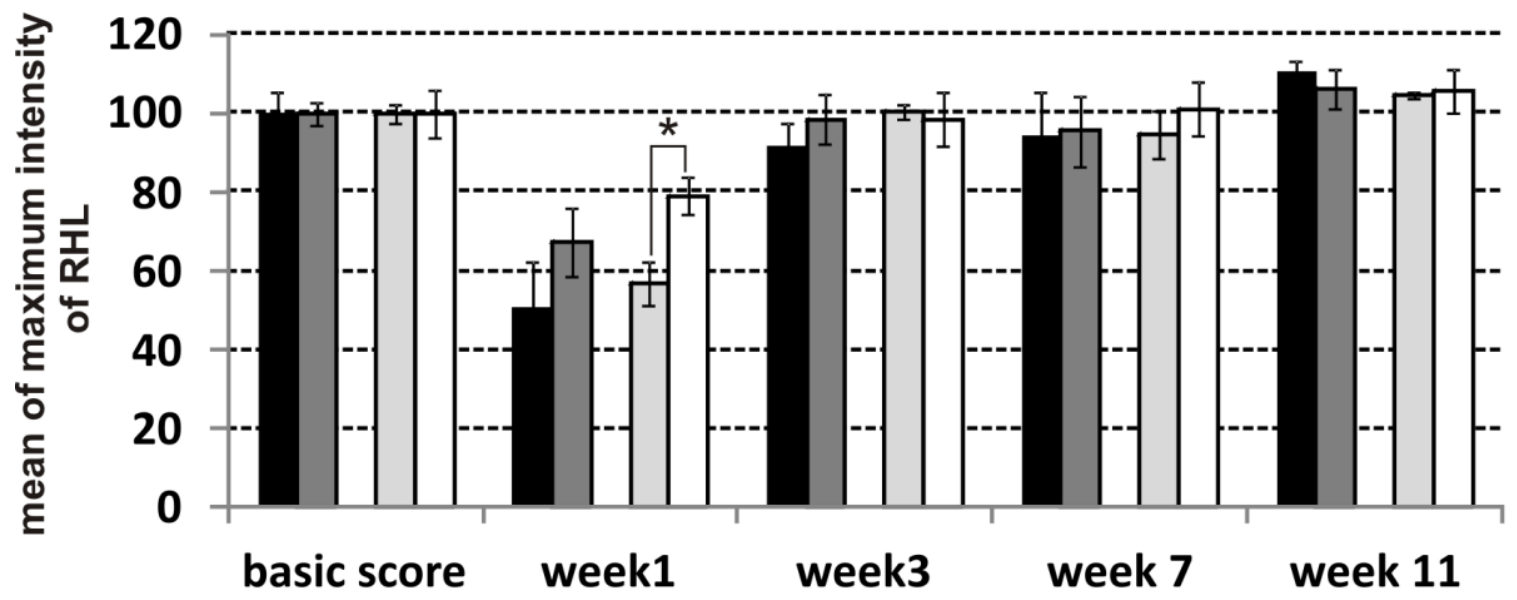

Figure 36. Effect of AAV.BAG1 and AAV.shROCK2 gene therapy on motor behavior quantified by CatWalk ${ }^{\mathrm{TM}}$ system. Results of the gait analysis using the CatWalk ${ }^{\mathrm{TM}}$ system displaying the mean maximum intensity of the foot print of the right hind limb (RHL). Means of three runs are taken for every animal. Bars represents the means \pm SEM. AAV.EGFP, $n=4$; AAV.BAG1, $n=8$, AAV.sh.firefly, $n=7$ \& AAV.sh.ROCK2, $n=8$. 


\section{Discussion}

Activation of growth impairing pathways by inhibitory extrinsic substrates, scar tissue and a markedly reduced intrinsic growth capacity of adult neurons lead to a severely impaired axonal regeneration, sprouting and functional recovery after spinal cord injury (SCl). Previous studies showed that an antiscarring treatment applying an iron chelator 2,2'- dipyridine-5, 5'-dicarboxylic acid (BPY-DCA) or 8-Br-cAMP (8-bromo-cyclic adenosine monophosphate) to the spinal lesion could ameliorate fibrous scarring and improve axonal regeneration in rats (Klapka, Hermanns et al. 2005; Schiwy, Brazda et al. 2009). Other reports have reinforced neuronal survival and axonal re-growth using pharmacological ROCK inhibitors and AAV-vectors over-expressing BAG1 and reggie1 and demonstrated that both, neuronal survival and axonal regeneration were improved in rat optic nerve crush lesion models (Koch et al., 2013; Lingor et al., 2007; Planchamp et al., 2008). The present work assessed the potential of a combination therapy with AAV.BAG1 and antiscarring treatment using BPY-DCA on axonal sprouting and motor function 16 weeks after the dorsal spinal cord hemisection in rats. Next, the potential of AAV-mediated gene therapies overexpressing BAG1, reggie1 or miRNA-134 as well as downregulating ROCK2 (by shRNA) to promote restoration in a clinically relevant CNS lesion model, the dorsal spinal hemisection affecting the rubrospinal tract, was assessed.

\subsection{Effects of a combination therapy with AAV.BAG1 and AST at 16 weeks after dorsal spinal cord hemisection}

\subsubsection{Effects of anti-scarring treatment}

Scar tissue is a major barrier that impedes the growth of regeneration axons in the CNS and in order to facilitate axonal growth after lesion the scar tissue needs to be modulated to become more permissive. BPY-DCA is an ironchelator that inhibits prolyl-4-hydroxylase, which is a key molecule of collagen biosynthesis. Application of BPY-DCA in combination with 8-BrCAMP to the lesion showed a reduced scar and also improved axonal 
regeneration and motor behavior (Klapka, Hermanns et al. 2005; Schiwy, Brazda et al. 2009). The studies of Klapka and Schiwy used 8-Br-cAMP in addition to BPY-DCA where they found significant axonal regeneration and locomotor improvement. Since our study represented a pilot experiment, we only used BPY-DCA as anti-scarring treatment, which might be less effective compared to the BPY-DCA \& 8-Br-cAMP combination. This is in line with previous findings, where BPY-DCA was used as single AST treatment and did not lead to reduced collagen scar in spinal cord lesions (Hermanns et al., 2001). Potentially, an anti-scarring regimen with 8-Br-cAMP and BPY-DCA in combination with potent gene therapies might be a better combination to enhance axonal regeneration.

\subsubsection{Effects of osmotic mini-pump implantation on lesion size}

Osmotic mini-pumps were used for continuous, effective delivery of pharmacological agents for a defined period of time to the target tissue. This technique has been shown to be effective in several studies for the intrathecal delivery of treatment agents to the spinal cord and brain (Klapka et al., 2005; Kobayashi et al., 1997; Wang et al., 2006). Osmotic mini-pump implantation was used to deliver the anti-scarring agent BPY-DCA (or TRIS buffer control) to the lesioned spinal cord in rats. In our study, larger lesion sizes were observed in animals treated with osmotic pump implantation compared to the animals without pump implantation. Similarly, scar formation around the catheter at 16 weeks after the implantation was also observed suggesting a possible reason for increased lesion size following pump implantation. Tissue damage has been previously reported in mice with brain infusions using osmotic mini-pump implantation (Jabłońska et al., 1993). Ruetenberg et al, 2002, also reported severe tissue damage and scar formation with continuous outflow of the fluid from osmotic mini-pumps. In some cases, the long placement of the catheter in our study lead to clogging of the cannula leading to an improper delivery of treatment to the target tissue. This might also explain the ineffectiveness of the BPY-DCA treatment on axonal regeneration along with the bigger lesion sizes affecting the treatment outcome of BPY-DCA on axonal sprouting. 
In spite of the limitations in animal numbers and increased lesion sizes, the pilot experiment suggested an increased sprouting responds for the AAV.BAG1-treated groups. In conclusion, the pilot trial yielded important information on the feasibility and limits of AAV-mediated gene therapy and application of AST via osmotic mini-pumps for the larger trial. Since the reason for pump-related lesion size increase could not be finally determined, we omitted this step in further experimental setups and switched to an adapted protocol with only AAV-mediated treatments.

\subsection{Optimization of anterograde RST tracing}

Anterograde tracing of the axons is a "gold standard" for assessing axonal regeneration in spinal cord lesion models. BDA and CTB are among the most commonly employed anterograde tracers for the descending spinal cord tracts used in many studies on spinal cord injury (Hagg et al., 2005; Hellenbrand et al., 2013; Reiner et al., 2000).

In our paradigm, anterograde tracing with BDA could not sufficiently trace the RST after the lesion. Injections of AAV.dsRed into the RN, however, transduced the RST at least at high cervical levels. Anterograde tracing with RN-injections of AAV.EGFP immediately after the SC hemisection sufficiently labeled the RST at cervical and thoracic levels proximal to the lesion.

RN neurons were reported to undergo atrophy and cell shrinkage after spinal cord lesions (Kwon et al., 2004; Tetzlaff et al., 1991). One explanation for the failure of BDA and CTB labeling could be the limited uptake of these dyes by atrophied RN neurons. On the other hand, the injection of AAV at the time of lesion was much more successful. Here, the RN neurons were not yet atrophied and fluorophore expression was thus not impaired.

For further experiments, we thus chose the strategy of simultaneous injection of AAV-vectors immediately following the spinal cord lesion. That way, we could (1) assure a proper labeling of the lesioned RST and (2) express our 
targets of interest in a postlesional setting, mimicking the real therapeutic challenge present in SCI patients.

\subsection{Effects of gene therapy with AAV.BAG1 and AAV.reggie1, AAV.miRNA-134 and ROCK2.sh.RNA}

\subsubsection{Toxic effects of AAV.miRNA-134 and AAV.reggie1 expression}

RNA interference (RNAi) mediated gene silencing is a powerful tool for knockdown of target genes. In the CNS, the blood brain barrier is a major limitation for systemic delivery of RNAi for chronic treatments (Meister and Tuschl, 2004; Scherer and Rossi, 2003). For the safe and effective delivery of the RNAi, AAV vectors expressing short-hairpin RNA (shRNA) or microRNA (miRNA) have been employed (Hommel et al., 2003; Ulusoy et al., 2009). In this study, we aimed to evaluate the effects of miRNA-134 on neuroprotection and axonal sprouting after the dorsal spinal hemisection in rats. Brain specific miRNA-134 expression increased dendrite spine stability by regulation of LIMK1 via cofilin and BDNF (Numakawa et al., 2011; Schratt et al., 2006). However, in the present study expression of miRNA-134 led to toxic effects in the RN resulting in decreased neuronal numbers. In contrast to paradigms, where miRNAs are expressed for a short period of time, our experimental model required the expression for 12 weeks. One reason for the observed AAV-vector toxicity could thus be the chronic expression of neurons to levels of miRNA-134, which are markedly higher than normal. This can result in a saturation of the endogenous miRNA-processing machinery, which could prevent other miRNA from being processed and thus lead to metabolic dysregulation. Another possibility could be an increased selective vulnerability of RN neurons. Previously, dose-mediate toxic effects were reported with viral-mediated RNAi in liver and brain tissue (Ehlert et al., 2010; Grimm, 2011; Grimm et al., 2006).

Unexpectedly, we observed rather pronounced toxic effects with another viral vector used in this study, the AAV.reggie1-vector. Reggie1 expression was reported to increase axonal regeneration in fish as well as in CNS (Stuermer, 2010). In preparation to this work, data from our lab suggested that over- 
expression of reggie1 increased axonal regeneration in the optic nerve crush model (Koch et al., 2013). In this paradigm, AAV-mediated expression was used to overexpress the target protein for duration of 6 weeks and the targeted population was retinal ganglion cells. No obvious toxicity was observed in this experimental setup, which, however, was significantly shorter and targeted a different neuronal subtype.

In conclusion, due to rather pronounced toxic effects induced by both AAV.miRNA-134 and AAV.reggie1 vectors, we decided to exclude these animals from further analysis. Excess levels of miRNA, which cannot be regulated with the constitutive expression from our vector system, the duration of target expression and the infected neuronal subtype, i.e. RN neurons, could all contribute to increased toxicity, which was observed in our study.

\subsubsection{Effects of AAV.BAG1 and AAV.sh.ROCK2 on RN neuronal survival}

Red nucleus neurons were reported to undergo profound cell atrophy, perikaryotic shrinkage and also retrograde cell death after rubrospinal tract injury (Kwon et al., 2004; Tetzlaff et al., 1991). However, studies on neuronspecific application of growth factors, such as brain derived neurotropic factor (BDNF) also reported successful reversal of neuronal atrophy after dorsal hemisection (Ruitenberg et al., 2004). Since BAG1 conferred neuroprotective effects in models of neurovascular and neurodegenerative diseases (Jana and Nukina, 2005; Sroka et al., 2009), its ability to counteract lesion-induced neuronal atrophy was tested. The neuroprotective effects of BAG1 are known to be mediated not only via Hsp70, Bcl-2, Raf-1 and ERK, but also via inhibition of ROCK, which also a known to be neuroprotective in various lesion paradigms (Planchamp et al., 2008). Thus, both AAV therapies employed in this study were expected to beneficially affect neuronal atrophy. Indeed, a significant $\sim 2$-fold increase of RN neuronal counts was observed with neuron-specific overexpression of AAV.BAG1 and AAV.sh.ROCK2. AAV.BAG1 also increased the number of neurons with larger somata underscoring its neuroprotective effect. Although AAV.sh.ROCK2 also 
increased the number of $\mathrm{RN}$ neurons by $\sim 2$-fold as compared to the appropriate AAV.sh.firefly control, values in both shRNA-expressing vector groups were generally lower than in the protein-overexpressing AAV groups. One possible explanation for this could be the shRNA-mediated toxicity to the $\mathrm{RN}$, possibly by overload of the intracellular miRNA-processing machinery (Ehlert et al., 2010).

\subsubsection{Effects of AAV.BAG1 and AAV.sh.ROCK2 on axonal sprouting and axonal regeneration}

While increased neuronal survival is a prerequisite for successful restoration, functional restitution requires the establishment of axonal projection either by sprouting or by regenerative growth. Collateral axonal sprouting at cervical level proximal to the lesion after thoracic hemisection was reported in earlier studies, e.g. where BDNF was applied to the cell bodies (Fouad et al., 2001; Vavrek et al., 2006). It represents a compensatory mechanism promoting the adaptive response for the recovery of motor function after SCI. Neuronspecific overexpression of BAG1 and shRNA-mediated downregulation of ROCK2 significantly enhanced the axonal sprouting at both cervical and thoracic levels compared to their corresponding controls. Interestingly, AAV.sh.ROCK2-treated animals showed a 10-fold increase in sprouting fibres compared to the control shRNA-vector, while AAV.BAG1 improved the sprouting response by the factor of $\sim 2$. Disinhibition of myelin-induced growth impairment is a major prerequisite for an effective sprouting response (Tanaka et al., 2004) and AAV-mediated ROCK2-inhibition acts at exactly this point. Although BAG1 has been shown to regulate ROCK activity (Planchamp et al., 2008) this effect, in relation, seems less effective than the targeted downregulation of ROCK2.

In contrast to the pronounced neuroprotective effects and increased cervical and thoracic sprouting, none of the animals showed a regeneration of EGFPpositive axons into or even across the lesion site. True axonal regeneration into the lesion site depends not only on the regulation of inhibitory substrates, but requires a strong activation of intrinsic growth signals, e.g. as was recently demonstrated by deletion of PTEN and/or SOCS3 (Sun et al., 2011). 
BAG1 and ROCK2-inhibition enhance intrinsic growth pathways, e.g. MAPK/ERK (Planchamp et al., 2008). However, this alone appears insufficient to induce a true regenerative axonal growth and thus would call for combinatorial therapeutic approaches (Lingor et al., 2008).

\subsubsection{Effects of AAV.BAG1 and AAV.sh.ROCK2 on axonal degeneration and lesion size}

Stabilization of axons proximal to the lesion, e.g. by application of taxol, has been shown to attenuate retraction bulb formation and to promote the regenerative capacity of lesioned axons (Ertürk et al., 2007). Therefore, we assessed the potential of AAV gene therapy to stabilize RST axons. Although, an increase of remaining RST axons proximal to the lesion site in the AAV.BAG1-treated group is a common trend, the effects on axonal stability were marginal. In vitro, ROCK inhibition was previously shown to reduce axonal degeneration following lesion (Gallo, 2004) which can be due to a decreased speed of microtubule depolymerization. However, this been effective on a short term but fails to yield sustained results in a 12-week lesion paradigm as in our case. Thus, the present study suggests that prolonged axonal stabilization requires the targeting of additional mechanisms, e.g. modulation of axonal autophagy (Hao et al., 2013; Kanno et al., 2011), which are only insufficiently addressed by AAV therapy.

The lesion area per se was unaffected by whatever AAV-vector was used and all animals included in the study showed a continuity disruption as well as a cyst formation at the lesion site. This comes with no surprise, since the approach in the final experiment was not directed to target local scar formation. Unfortunately, manipulation of the lesion size by AST, as initially envisioned, failed due to complications of the osmotic mini-pump therapy.

\subsubsection{Effects of AAV.BAG1 and AAV.sh.ROCK2 on motor recovery}

Rubrospinal tract injury results in functional deficits of the limbs affecting the step pattern and weight bearing ability in rats (Webb and Muir, 2003), (Hendriks et al., 2006). The current study investigated the motor 
improvement of the animals with the BBB open field test. To increase the sensitivity, specific parameters such as trunk instability, toe clearance and paw placement were assessed as sub score. In correlation to the most pronounced improvement in neuronal survival and collateral sprouting, a significant improvement in the sub score was observed in the AAV.BAG1 treated group. AAV.sh.ROCK2-treated animals showed a trend for motor improvement, but didn't reach statistical significance and this correlated with less important improvements in the histological parameters in this treatment group.

\subsection{Conclusion and perspectives}

Regeneration and functional restoration after spinal cord injury (SCl) largely depends on neuroprotection and the targeting of inhibitory pathways. In order to restore SC function, it is thus crucial to simultaneously attenuate inhibitory signaling and promote neuroprotection. Here, we investigated AAV-mediated gene therapy with various strategies: over-expression of BAG1, reggie1, and miRNA-134 and downregulation of ROCK2 via shRNA. All of these molecular targets aim at the increase of neuronal survival and/or disinhibition of key inhibitory pathways for axonal growth. Our studies clearly demonstrate that not only the choice of the target molecule, but also its AAV-vector-mediated expression itself can have a significant influence on the biological outcome.

Due to the complexity of SC injury pathophysiology it is very likely that combinational studies with synergistic effects will be used in future approaches. For example, combination treatments with neurotrophic factors, chondroitinase ABC (a bacterial enzyme degrading CSPGs), cell therapies and molecules interacting with inhibitory pathways gained much attention in recent years. For example, Pearse et.al showed that a combination of cAMP and Schwann cells promote axonal regeneration and motor function recovery after spinal cord lesion in rats in (Pearse et al., 2004). A combination of Nogo-A antibody and chondroitinase $A B C$ also showed effects on functional recovery and this was more pronounced than the single treatment (Zhao et al., 2013). Targeting pathways other than Rho-ROCK, such semaphorins, ephrins and autophagy could also broaden the potential treatment armoire. 
Recently, robust axonal regeneration and sprouting was obtained by modulating the PTEN/mTOR pathway after spinal cord injury in mice (Liu et al., 2010). Taken together, combination therapies could be important to achieve more pronounced beneficial effects after the spinal cord injury and our study yields two interesting targets, which are worthwhile to be explored in such therapies: BAG1 and ROCK2.

\section{Summary and conclusions}

The rat rubrospinal tract lesion is an established model for traumatic spinal cord injury, which results in atrophy of the red nucleus neurons, axonal dieback and locomotor deficits.

1. In the pilot experiment, a combination of AAV.BAG1 and BPY-DCA antiscarring treatment in a rat model of unilateral dorsal spinal cord lesion was tested. Animals were followed up for 16 weeks. A trend to increased sprouting was observed in the AAV.BAG1-treated animals, but there was no significant functional improvement by the treatment. On the contrary, we observed increased lesion sizes after implantation of osmotic minipumps in the spinal cord.

2. Anterograde tracing experiments with BDA and CTB suggested that the atrophied RN neurons were unable to take up the dye after the lesion and injections of AAV-vectors immediately after $\mathrm{SCl}$ were more effective for the labelling of the RST.

3. In the final experiment, AAV gene therapy was used to overexpress BAG1, reggie1, miRNA-134 and ROCK2-shRNA in the red nucleus in order to trace (by co-expression of EGFP) and treat the rubrospinal tract after unilateral dorsal hemisection. Toxic effects were observed by expression of AAV.miRNA-134 and AAV.reggie1. However, expression of BAG1 and ROCK2-shRNA promoted neuronal survival of red nucleus neurons and enhanced axonal sprouting proximal to the lesion. Only marginal effects were observed on locomotor function. 
Taken together, this study implies that AAV-mediated gene therapy is a highly interesting future therapeutic tool for the treatment of traumatic CNS injuries. BAG1 represents a valuable target, stimulating neuronal survival and collateral sprouting. Although BAG1 expression is able to down-regulate ROCK activity, additional pathways likely mediate its beneficial effects in the present model, since ROCK2 down-regulation alone was less powerful. Interestingly, shRNA-mediated down-regulation of ROCK2 appears to have a stronger effect on axonal sprouting, which could be explained by only partial ROCK2 inhibition following BAG1 overexpression. Future studies will have to address the value of both targets in combination treatments, which should also include options for the modulation of the scar formation. 


\section{References}

Anderson, C.R., Ashwell, K.W., Collewijn, H., Conta, A., Harvey, A., Heise, C., Hodgetts, S., Holstege, G., Kayalioglu, G., Keast, J.R., et al. (2009). The Spinal Cord: A Christopher and Dana Reeve Foundation Text and Atlas. In The Spinal Cord, C. Watson, G. Paxinos, and G. Kayalioglu, eds. (San Diego: Academic Press), p. v.

Arber, S., Barbayannis, F.A., Hanser, H., Schneider, C., Stanyon, C.A., Bernard, O., and Caroni, P. (1998). Regulation of actin dynamics through phosphorylation of cofilin by LIM-kinase. Nature $393,805-809$.

Azzouz, M., Hottinger, A., Paterna, J.C., Zurn, A.D., Aebischer, P., and Büeler, H. (2000). Increased motoneuron survival and improved neuromuscular function in transgenic ALS mice after intraspinal injection of an adeno-associated virus encoding Bcl-2. Hum. Mol. Genet. 9, 803-811.

Basso, D.M., Beattie, M.S., and Bresnahan, J.C. (1995). A sensitive and reliable locomotor rating scale for open field testing in rats. J. Neurotrauma $12,1-21$.

Benson, M.D., Romero, M.I., Lush, M.E., Lu, Q.R., Henkemeyer, M., and Parada, L.F. (2005). Ephrin-B3 is a myelin-based inhibitor of neurite outgrowth. Proc. Natl. Acad. Sci. U. S. A. 102, 10694-10699.

Bickel, P.E., Scherer, P.E., Schnitzer, J.E., Oh, P., Lisanti, M.P., and Lodish, H.F. (1997). Flotillin and Epidermal Surface Antigen Define a New Family of Caveolae-associated Integral Membrane Proteins. J. Biol. Chem. 272, 13793-13802.

Blits, B., Oudega, M., Boer, G.J., Bartlett Bunge, M., and Verhaagen, J. (2003). Adeno-associated viral vector-mediated neurotrophin gene transfer in the injured adult rat spinal cord improves hind-limb function. Neuroscience $118,271-281$.

Blits, B., Carlstedt, T.P., Ruitenberg, M.J., de Winter, F., Hermens, W.T.J.M.C., Dijkhuizen, P.A., Claasens, J.W.C., Eggers, R., van der Sluis, R., Tenenbaum, L., et al. (2004). Rescue and sprouting of motoneurons following ventral root avulsion and reimplantation combined with intraspinal adeno-associated viral vector-mediated expression of glial cell line-derived neurotrophic factor or brain-derived neurotrophic factor. Exp. Neurol. 189, 303-316.

Blits, B., Derks, S., Twisk, J., Ehlert, E., Prins, J., and Verhaagen, J. (2010). Adeno-associated viral vector (AAV)-mediated gene transfer in the red nucleus of the adult rat brain: comparative analysis of the transduction properties of seven AAV serotypes and lentiviral vectors. J. Neurosci. Methods $185,257-263$.

Brown, L.T. (1974). Rubrospinal projections in the rat. J. Comp. Neurol. 154, 169-187. 
Buchli, A.D., and Schwab, M.E. (2005). Inhibition of Nogo: a key strategy to increase regeneration, plasticity and functional recovery of the lesioned central nervous system. Ann. Med. 37, 556-567.

Buller, R.M., Janik, J.E., Sebring, E.D., and Rose, J.A. (1981). Herpes simplex virus types 1 and 2 completely help adenovirus-associated virus replication. J. Virol. 40, 241-247.

Bunge, R.P., Puckett, W.R., Becerra, J.L., Marcillo, A., and Quencer, R.M. (1993). Observations on the pathology of human spinal cord injury. A review and classification of 22 new cases with details from a case of chronic cord compression with extensive focal demyelination. Adv. Neurol. 59, 75-89.

Carter, L.M., McMahon, S.B., and Bradbury, E.J. (2011). Delayed treatment with chondroitinase $A B C$ reverses chronic atrophy of rubrospinal neurons following spinal cord injury. Exp. Neurol. 228, 149-156.

Coleman, M.P., and Perry, V.H. (2002). Axon pathology in neurological disease: a neglected therapeutic target. Trends Neurosci. 25, 532-537.

Crowe, M.J., Bresnahan, J.C., Shuman, S.L., Masters, J.N., and Crowe, M.S. (1997). Apoptosis and delayed degeneration after spinal cord injury in rats and monkeys. Nat. Med. 3, 73-76.

Daya, S., and Berns, K.I. (2008). Gene Therapy Using Adeno-Associated Virus Vectors. Clin. Microbiol. Rev. 21, 583-593.

Dergham, P., Ellezam, B., Essagian, C., Avedissian, H., Lubell, W.D., and McKerracher, L. (2002). Rho signaling pathway targeted to promote spinal cord repair. J. Neurosci. Off. J. Soc. Neurosci. 22, 6570-6577.

Donnelly, D.J., and Popovich, P.G. (2008). Inflammation and its role in neuroprotection, axonal regeneration and functional recovery after spinal cord injury. Exp. Neurol. 209, 378-388.

Doucette, T.A., Ryan, C.L., and Tasker, R.A. (2000). Use of osmotic minipumps for sustained drug delivery in rat pups: effects on physical and neurobehavioural development. Physiol. Behav. 71, 207-212.

Ehlers, M.D. (2004). Deconstructing the axon: Wallerian degeneration and the ubiquitin-proteasome system. Trends Neurosci. 27, 3-6.

Ehlert, E.M., Eggers, R., Niclou, S.P., and Verhaagen, J. (2010). Cellular toxicity following application of adeno-associated viral vector-mediated RNA interference in the nervous system. BMC Neurosci. 11, 20.

Emery, E., Aldana, P., Bunge, M.B., Puckett, W., Srinivasan, A., Keane, R.W., Bethea, J., and Levi, A.D. (1998). Apoptosis after traumatic human spinal cord injury. J. Neurosurg. 89, 911-920.

Ertürk, A., Hellal, F., Enes, J., and Bradke, F. (2007). Disorganized microtubules underlie the formation of retraction bulbs and the failure of axonal regeneration. J. Neurosci. Off. J. Soc. Neurosci. 27, 9169-9180. 
Fawcett, J.W., Housden, E., Smith-Thomas, L., and Meyer, R.L. (1989). The growth of axons in three-dimensional astrocyte cultures. Dev. Biol. 135, 449458.

Fehlings, M.G., Theodore, N., Harrop, J., Maurais, G., Kuntz, C., Shaffrey, C.I., Kwon, B.K., Chapman, J., Yee, A., Tighe, A., et al. (2011). A phase Vlla clinical trial of a recombinant Rho protein antagonist in acute spinal cord injury. J. Neurotrauma 28, 787-796.

Filli, L., and Schwab, M.E. (2012). The rocky road to translation in spinal cord repair. Ann. Neurol. 72, 491-501.

Forgione, N., and Fehlings, M.G. (2013). Rho-ROCK Inhibition in the Treatment of Spinal Cord Injury. World Neurosurg.

Fouad, K., Pedersen, V., Schwab, M.E., and Brösamle, C. (2001). Cervical sprouting of corticospinal fibers after thoracic spinal cord injury accompanies shifts in evoked motor responses. Curr. Biol. 11, 1766-1770.

Frisén, J., Fried, K., Sjögren, A.M., and Risling, M. (1993). Growth of ascending spinal axons in CNS scar tissue. Int. J. Dev. Neurosci. Off. J. Int. Soc. Dev. Neurosci. 11, 461-475.

Gage, G.J., Kipke, D.R., and Shain, W. (2012). Whole Animal Perfusion Fixation for Rodents. J. Vis. Exp. JoVE.

Gallo, G. (2004). Myosin II activity is required for severing-induced axon retraction in vitro. Exp. Neurol. 189, 112-121.

García-Alías, G., Lin, R., Akrimi, S.F., Story, D., Bradbury, E.J., and Fawcett, J.W. (2008). Therapeutic time window for the application of chondroitinase ABC after spinal cord injury. Exp. Neurol. 210, 331-338.

Geffner, L.F., Santacruz, P., Izurieta, M., Flor, L., Maldonado, B., Auad, A.H., Montenegro, X., Gonzalez, R., and Silva, F. (2008). Administration of Autologous Bone Marrow Stem Cells Into Spinal Cord Injury Patients Via Multiple Routes Is Safe and Improves Their Quality of Life: Comprehensive Case Studies. Cell Transplant. 17, 1277-1293.

Gemma, M., Perego, G.B., Pizzini, G., and Tredici, G. (1987). Distribution of the cortico-spinal fibres in the cervical and lumbar enlargements of the rat spinal cord. J. Für Hirnforsch. 28, 457-462.

Giger, R.J., Hollis, E.R., 2nd, and Tuszynski, M.H. (2010). Guidance molecules in axon regeneration. Cold Spring Harb. Perspect. Biol. 2, a001867.

Goldshmit, Y., Galea, M.P., Wise, G., Bartlett, P.F., and Turnley, A.M. (2004). Axonal regeneration and lack of astrocytic gliosis in EphA4-deficient mice. J. Neurosci. Off. J. Soc. Neurosci. 24, 10064-10073.

Götz, R., Wiese, S., Takayama, S., Camarero, G.C., Rossoll, W., Schweizer, U., Troppmair, J., Jablonka, S., Holtmann, B., Reed, J.C., et al. (2005). Bag1 
is essential for differentiation and survival of hematopoietic and neuronal cells. Nat. Neurosci. 8, 1169-1178.

Grimm, D. (2011). The dose can make the poison: lessons learned from adverse in vivo toxicities caused by RNAi overexpression. Silence 2, 8.

Grimm, D., Streetz, K.L., Jopling, C.L., Storm, T.A., Pandey, K., Davis, C.R., Marion, P., Salazar, F., and Kay, M.A. (2006). Fatality in mice due to oversaturation of cellular microRNA/short hairpin RNA pathways. Nature 441, 537-541.

Guest, J., Santamaria, A.J., and Benavides, F.D. (2013). Clinical translation of autologous Schwann cell transplantation for the treatment of spinal cord injury. Curr. Opin. Organ Transplant. 18, 682-689.

Hagg, T., and Oudega, M. (2006). Degenerative and Spontaneous Regenerative Processes after Spinal Cord Injury. J. Neurotrauma 23, 263280.

Hagg, T., Baker, K.A., Emsley, J.G., and Tetzlaff, W. (2005). Prolonged local neurotrophin-3 infusion reduces ipsilateral collateral sprouting of spared corticospinal axons in adult rats. Neuroscience $130,875-887$.

Hamers, F.P., Lankhorst, A.J., van Laar, T.J., Veldhuis, W.B., and Gispen, W.H. (2001). Automated quantitative gait analysis during overground locomotion in the rat: its application to spinal cord contusion and transection injuries. J. Neurotrauma 18, 187-201.

Hao, H.-H., Wang, L., Guo, Z.-J., Bai, L., Zhang, R.-P., Shuang, W.-B., Jia, Y.-J., Wang, J., Li, X.-Y., and Liu, Q. (2013). Valproic acid reduces autophagy and promotes functional recovery after spinal cord injury in rats. Neurosci. Bull. 29, 484-492.

Hashimoto, R., Nakamura, Y., Kosako, H., Amano, M., Kaibuchi, K., Inagaki, M., and Takeda, M. (1999). Distribution of Rho-kinase in the bovine brain. Biochem. Biophys. Res. Commun. 263, 575-579.

Hayes, K.C., Davies, A.L., Ashki, N., Kramer, J.K., and Close, T.E. (2007). Re: Ditunno JF, Little JW, Tessler A, Burns AS. Spinal shock revisited: a four-phase model. Spinal Cord 2004; 42: 383-395. Spinal Cord 45, 395-396.

Hellal, F., Hurtado, A., Ruschel, J., Flynn, K.C., Laskowski, C.J., Umlauf, M., Kapitein, L.C., Strikis, D., Lemmon, V., Bixby, J., et al. (2011). Microtubule stabilization reduces scarring and causes axon regeneration after spinal cord injury. Science 331, 928-931.

Hellenbrand, D.J., Kaeppler, K.E., Hwang, E., Ehlers, M.E., Toigo, R.D., Giesler, J.D., Vassar-Olsen, E.R., and Hanna, A. (2013). Basic techniques for long distance axon tracing in the spinal cord. Microsc. Res. Tech. 76, 1240-1249.

Hendriks, W.T.J., Eggers, R., Ruitenberg, M.J., Blits, B., Hamers, F.P.T., Verhaagen, J., and Boer, G.J. (2006). Profound differences in spontaneous 
long-term functional recovery after defined spinal tract lesions in the rat. J. Neurotrauma 23, 18-35.

Hermanns, S., Reiprich, P., and Müller, H.W. (2001). A reliable method to reduce collagen scar formation in the lesioned rat spinal cord. J. Neurosci. Methods 110, 141-146.

Hill, C.E., Beattie, M.S., and Bresnahan, J.C. (2001). Degeneration and sprouting of identified descending supraspinal axons after contusive spinal cord injury in the rat. Exp. Neurol. 171, 153-169.

Hommel, J.D., Sears, R.M., Georgescu, D., Simmons, D.L., and DiLeone, R.J. (2003). Local gene knockdown in the brain using viral-mediated RNA interference. Nat. Med. 9, 1539-1544.

Jabłońska, B., Gierdalski, M., Kublik, A., Skangiel-Kramska, J., and Kossut, M. (1993). Effects of implantation of Alzet 1007D osmotic minipumps upon 2deoxyglucose uptake in the cerebral cortex of mice. Acta Neurobiol. Exp. (Warsz.) 53, 577-580.

James W. Rowland, Gregory W. J. Hawryluk, Brian Kwon, and Michael G. Fehlings (2008). Current status of acute spinal cord injury pathophysiology and emerging therapies: promise on the horizon.

Jana, N.R., and Nukina, N. (2005). BAG-1 associates with the polyglutamineexpanded huntingtin aggregates. Neurosci. Lett. 378, 171-175.

Kanno, H., Ozawa, H., Sekiguchi, A., Yamaya, S., and Itoi, E. (2011). Induction of autophagy and autophagic cell death in damaged neural tissue after acute spinal cord injury in mice. Spine 36, E1427-1434.

Kaplitt, M.G., Leone, P., Samulski, R.J., Xiao, X., Pfaff, D.W., O’Malley, K.L., and During, M.J. (1994). Long-term gene expression and phenotypic correction using adeno-associated virus vectors in the mammalian brain. Nat. Genet. 8, 148-154.

Kastin, A.J., and Pan, W. (2005). Targeting neurite growth inhibitors to induce CNS regeneration. Curr. Pharm. Des. 11, 1247-1253.

Kermer, P., Krajewska, M., Zapata, J.M., Takayama, S., Mai, J., Krajewski, S., and Reed, J.C. (2002). Bag1 is a regulator and marker of neuronal differentiation. Cell Death Differ. 9, 405-413.

Kerschensteiner, M., Schwab, M.E., Lichtman, J.W., and Misgeld, T. (2005). In vivo imaging of axonal degeneration and regeneration in the injured spinal cord. Nat. Med. 11, 572-577.

Kiyota, T., Yamamoto, M., Schroder, B., Jacobsen, M.T., Swan, R.J., Lambert, M.P., Klein, W.L., Gendelman, H.E., Ransohoff, R.M., and Ikezu, T. (2009). AAV1/2-mediated CNS gene delivery of dominant-negative CCL2 mutant suppresses gliosis, beta-amyloidosis, and learning impairment of APP/PS1 mice. Mol. Ther. J. Am. Soc. Gene Ther. 17, 803-809. 
Klapka, N., Hermanns, S., Straten, G., Masanneck, C., Duis, S., Hamers, F.P.T., Müller, D., Zuschratter, W., and Müller, H.W. (2005). Suppression of fibrous scarring in spinal cord injury of rat promotes long-distance regeneration of corticospinal tract axons, rescue of primary motoneurons in somatosensory cortex and significant functional recovery. Eur. J. Neurosci. 22, 3047-3058.

Knoller, N., Auerbach, G., Fulga, V., Zelig, G., Attias, J., Bakimer, R., Marder, J.B., Yoles, E., Belkin, M., Schwartz, M., et al. (2005). Clinical experience using incubated autologous macrophages as a treatment for complete spinal cord injury: phase I study results. J. Neurosurg. Spine 3, 173-181.

Kobayashi, N.R., Fan, D.P., Giehl, K.M., Bedard, A.M., Wiegand, S.J., and Tetzlaff, W. (1997). BDNF and NT-4/5 prevent atrophy of rat rubrospinal neurons after cervical axotomy, stimulate GAP-43 and Talpha1-tubulin mRNA expression, and promote axonal regeneration. J. Neurosci. Off. J. Soc. Neurosci. 17, 9583-9595.

Koch, J.C., Barski, E., Lingor, P., Bähr, M., and Michel, U. (2011). Plasmids containing NRSE/RE1 sites enhance neurite outgrowth of retinal ganglion cells via sequestration of REST independent of NRSE dsRNA expression. FEBS J. 278, 3472-3483.

Koch, J.C., Solis, G.P., Bodrikov, V., Michel, U., Haralampieva, D., Shypitsyna, A., Tönges, L., Bähr, M., Lingor, P., and Stuermer, C.A.O. (2013). Upregulation of reggie-1/flotillin-2 promotes axon regeneration in the rat optic nerve in vivo and neurite growth in vitro. Neurobiol. Dis. 51, 168176.

Koda, M., Hashimoto, M., Murakami, M., Yoshinaga, K., Ikeda, O., Yamazaki, M., Koshizuka, S., Kamada, T., Moriya, H., Shirasawa, H., et al. (2004). Adenovirus vector-mediated in vivo gene transfer of brain-derived neurotrophic factor (BDNF) promotes rubrospinal axonal regeneration and functional recovery after complete transection of the adult rat spinal cord. J. Neurotrauma 21, 329-337.

Komagome, R., Kimura, K., and Saito, M. (2000). Postnatal changes in Rho and Rho-related proteins in the mouse brain. Jpn. J. Vet. Res. 47, 127-133.

Küchler, M., Fouad, K., Weinmann, O., Schwab, M.E., and Raineteau, O. (2002). Red nucleus projections to distinct motor neuron pools in the rat spinal cord. J. Comp. Neurol. 448, 349-359.

Kügler, S., Meyn, L., Holzmüller, H., Gerhardt, E., Isenmann, S., Schulz, J.B., and Bähr, M. (2001). Neuron-specific expression of therapeutic proteins: evaluation of different cellular promoters in recombinant adenoviral vectors. Mol. Cell. Neurosci. 17, 78-96.

Kundi (2013). SPINAL CORD INJURY: CURRENT MAMMALIAN MODELS. Am. J. Neurosci. 4, 1-12. 
Kwon, B.K., Liu, J., Messerer, C., Kobayashi, N.R., McGraw, J., Oschipok, L., and Tetzlaff, W. (2002a). Survival and regeneration of rubrospinal neurons 1 year after spinal cord injury. Proc. Natl. Acad. Sci. U. S. A. 99, 3246-3251.

Kwon, B.K., Liu, J., Oschipok, L., and Tetzlaff, W. (2002b). Reaxotomy of Chronically Injured Rubrospinal Neurons Results in Only Modest Cell Loss. Exp. Neurol. 177, 332-337.

Kwon, B.K., Liu, J., Oschipok, L., Teh, J., Liu, Z.W., and Tetzlaff, W. (2004). Rubrospinal neurons fail to respond to brain-derived neurotrophic factor applied to the spinal cord injury site 2 months after cervical axotomy. Exp. Neurol. 189, 45-57.

Kwon, B.K., Liu, J., Lam, C., Plunet, W., Oschipok, L.W., Hauswirth, W., Di Polo, A., Blesch, A., and Tetzlaff, W. (2007). Brain-derived neurotrophic factor gene transfer with adeno-associated viral and lentiviral vectors prevents rubrospinal neuronal atrophy and stimulates regenerationassociated gene expression after acute cervical spinal cord injury. Spine 32, 1164-1173.

Langhorst, M.F., Jaeger, F.A., Mueller, S., Sven Hartmann, L., Luxenhofer, G., and Stuermer, C.A.O. (2008). Reggies/flotillins regulate cytoskeletal remodeling during neuronal differentiation via CAP/ponsin and Rho GTPases. Eur. J. Cell Biol. 87, 921-931.

Lehmann, M., Fournier, A., Selles-Navarro, I., Dergham, P., Sebok, A., Leclerc, N., Tigyi, G., and McKerracher, L. (1999). Inactivation of Rho signaling pathway promotes CNS axon regeneration. J. Neurosci. Off. J. Soc. Neurosci. 19, 7537-7547.

Lingor, P., Teusch, N., Schwarz, K., Mueller, R., Mack, H., Bähr, M., and Mueller, B.K. (2007). Inhibition of Rho kinase (ROCK) increases neurite outgrowth on chondroitin sulphate proteoglycan in vitro and axonal regeneration in the adult optic nerve in vivo. J. Neurochem. 103, 181-189.

Lingor, P., Tönges, L., Pieper, N., Bermel, C., Barski, E., Planchamp, V., and Bähr, M. (2008). ROCK inhibition and CNTF interact on intrinsic signalling pathways and differentially regulate survival and regeneration in retinal ganglion cells. Brain J. Neurol. 131, 250-263.

Liu, K., Lu, Y., Lee, J.K., Samara, R., Willenberg, R., Sears-Kraxberger, I., Tedeschi, A., Park, K.K., Jin, D., Cai, B., et al. (2010). PTEN deletion enhances the regenerative ability of adult corticospinal neurons. Nat. Neurosci. 13, 1075-1081.

Liu, Y., Kim, D., Himes, B.T., Chow, S.Y., Schallert, T., Murray, M., Tessler, A., and Fischer, I. (1999). Transplants of fibroblasts genetically modified to express BDNF promote regeneration of adult rat rubrospinal axons and recovery of forelimb function. J. Neurosci. Off. J. Soc. Neurosci. 19, 43704387. 
Mackay-Sim, A., Féron, F., Cochrane, J., Bassingthwaighte, L., Bayliss, C., Davies, W., Fronek, P., Gray, C., Kerr, G., Licina, P., et al. (2008).

Autologous olfactory ensheathing cell transplantation in human paraplegia: a 3-year clinical trial. Brain J. Neurol. 131, 2376-2386.

Mandel, R.J., Spratt, S.K., Snyder, R.O., and Leff, S.E. (1997). Midbrain injection of recombinant adeno-associated virus encoding rat glial cell linederived neurotrophic factor protects nigral neurons in a progressive 6hydroxydopamine-induced degeneration model of Parkinson's disease in rats. Proc. Natl. Acad. Sci. U. S. A. 94, 14083-14088.

Matsui, T., Amano, M., Yamamoto, T., Chihara, K., Nakafuku, M., Ito, M., Nakano, T., Okawa, K., wamatsu, A., and Kaibuchi, K. (1996). Rhoassociated kinase, a novel serine/threonine kinase, as a putative target for small GTP binding protein Rho. EMBO J. 15, 2208-2216.

Mautes, A.E., Weinzierl, M.R., Donovan, F., and Noble, L.J. (2000). Vascular Events After Spinal Cord Injury: Contribution to Secondary Pathogenesis. Phys. Ther. 80, 673-687.

McCown, T.J. (2005). Adeno-associated virus (AAV) vectors in the CNS. Curr. Gene Ther. 5, 333-338.

McCown, T.J., Xiao, X., Li, J., Breese, G.R., and Samulski, R.J. (1996). Differential and persistent expression patterns of CNS gene transfer by an adeno-associated virus (AAV) vector. Brain Res. 713, 99-107.

McKerracher, L. (2001). Spinal cord repair: strategies to promote axon regeneration. Neurobiol. Dis. 8, 11-18.

De Medinaceli, L. (1986). An anatomical landmark for procedures on rat thoracic spinal cord. Exp. Neurol. 91, 404-408.

Meijering, E., Jacob, M., Sarria, J.-C.F., Steiner, P., Hirling, H., and Unser, M. (2004). Design and validation of a tool for neurite tracing and analys is in fluorescence microscopy images. Cytom. Part J. Int. Soc. Anal. Cytol. 58, 167-176.

Meister, G., and Tuschl, T. (2004). Mechanisms of gene silencing by doublestranded RNA. Nature 431, 343-349.

Metz, G.A., and Whishaw, I.Q. (2002). Cortical and subcortical lesions impair skilled walking in the ladder rung walking test: a new task to evaluate foreand hindlimb stepping, placing, and co-ordination. J. Neurosci. Methods 115, 169-179.

Metz, G.A., and Whishaw, I.Q. (2009). The ladder rung walking task: a scoring system and its practical application. J. Vis. Exp. JoVE.

Michel, U., Malik, I., Ebert, S., Bähr, M., and Kügler, S. (2005). Long-term in vivo and in vitro AAV-2-mediated RNA interference in rat retinal ganglion cells and cultured primary neurons. Biochem. Biophys. Res. Commun. 326, 307-312. 
Mueller, B.K., Mack, H., and Teusch, N. (2005). Rho kinase, a promising drug target for neurological disorders. Nat. Rev. Drug Discov. 4, 387-398.

Munderloh, C., Solis, G.P., Bodrikov, V., Jaeger, F.A., Wiechers, M., MálagaTrillo, E., and Stuermer, C.A.O. (2009). Reggies/Flotillins Regulate Retinal Axon Regeneration in the Zebrafish Optic Nerve and Differentiation of Hippocampal and N2a Neurons. J. Neurosci. 29, 6607-6615.

Nakagawa, O., Fujisawa, K., Ishizaki, T., Saito, Y., Nakao, K., and Narumiya, S. (1996). ROCK-I and ROCK-II, two isoforms of Rho-associated coiled-coil forming protein serine/threonine kinase in mice. FEBS Lett. 392, 189-193.

Norenberg, M.D., Smith, J., and Marcillo, A. (2004). The pathology of human spinal cord injury: defining the problems. J. Neurotrauma 21, 429-440.

Numakawa, T., Richards, M., Adachi, N., Kishi, S., Kunugi, H., and Hashido, K. (2011). MicroRNA function and neurotrophin BDNF. Neurochem. Int. 59, $551-558$.

Ohashi, K., Nagata, K., Maekawa, M., Ishizaki, T., Narumiya, S., and Mizuno, K. (2000). Rho-associated Kinase ROCK Activates LIM-kinase 1 by Phosphorylation at Threonine 508 within the Activation Loop. J. Biol. Chem. 275, 3577-3582.

Paxinos, G., and Watson, C. (1998). The Rat Brain: In Stereotaxic Coordinates (Academic Press, Incorporated).

Paxinos, G., Watson, C., Pennisi, M., and Topple, A. (1985). Bregma, lambda and the interaural midpoint in stereotaxic surgery with rats of different sex, strain and weight. J. Neurosci. Methods 13, 139-143.

Pearse, D.D., Pereira, F.C., Marcillo, A.E., Bates, M.L., Berrocal, Y.A., Filbin, M.T., and Bunge, M.B. (2004). cAMP and Schwann cells promote axonal growth and functional recovery after spinal cord injury. Nat. Med. 10,610616.

Peel, A.L., and Klein, R.L. (2000). Adeno-associated virus vectors: activity and applications in the CNS. J. Neurosci. Methods 98, 95-104.

Planchamp, V., Bermel, C., Tönges, L., Ostendorf, T., Kügler, S., Reed, J.C., Kermer, P., Bähr, M., and Lingor, P. (2008). BAG1 promotes axonal outgrowth and regeneration in vivo via Raf-1 and reduction of ROCK activity. Brain J. Neurol. 131, 2606-2619.

Rahimi-Movaghar, V. Saturated Picric Acid Prevents Autophagia and SelfMutilation in Laboratory Rats.

Reiner, A., Veenman, C.L., Medina, L., Jiao, Y., Del Mar, N., and Honig, M.G. (2000). Pathway tracing using biotinylated dextran amines. J. Neurosci.

Methods 103, 23-37.

Ren, K., Gao, C., Zhang, J., Wang, K., Xu, Y., Wang, S.-B., Wang, H., Tian, C., Shi, Q., and Dong, X.-P. (2013). Flotillin-1 mediates PrPc endocytosis in 
the cultured cells during $\mathrm{Cu}^{2+}$ stimulation through molecular interaction. Mol. Neurobiol. 48, 631-646.

Rolls, A., Shechter, R., and Schwartz, M. (2009). The bright side of the glial scar in CNS repair. Nat. Rev. Neurosci. 10, 235-241.

Rossignol, S., Schwab, M., Schwartz, M., and Fehlings, M.G. (2007). Spinal cord injury: time to move? J. Neurosci. Off. J. Soc. Neurosci. 27, 1178211792.

Rowland, J.W., Hawryluk, G.W.J., Kwon, B., and Fehlings, M.G. (2008). Current status of acute spinal cord injury pathophysiology and emerging therapies: promise on the horizon. Neurosurg. Focus 25, E2.

Ruitenberg, M.J., Eggers, R., Boer, G.J., and Verhaagen, J. (2002). Adenoassociated viral vectors as agents for gene delivery: application in disorders and trauma of the central nervous system. Methods San Diego Calif 28, 182194.

Ruitenberg, M.J., Blits, B., Dijkhuizen, P.A., te Beek, E.T., Bakker, A., van Heerikhuize, J.J., Pool, C.W., Hermens, W.T.J., Boer, G.J., and Verhaagen, J. (2004). Adeno-associated viral vector-mediated gene transfer of brainderived neurotrophic factor reverses atrophy of rubrospinal neurons following both acute and chronic spinal cord injury. Neurobiol. Dis. 15, 394-406.

Samuel, F., and Hynds, D.L. (2010). RHO GTPase Signaling for Axon Extension: Is Prenylation Important? Mol. Neurobiol. 42, 133-142.

Santiago, J.M., Torrado, A.I., Arocho, L.C., Rosas, O.R., Rodríguez, A.E., Toro, F.K., Salgado, I.K., Torres, Y.A., Silva, W.I., and Miranda, J.D. (2013). Expression profile of flotillin-2 and its pathophysiological role after spinal cord injury. J. Mol. Neurosci. MN 49, 347-359.

Scherer, L.J., and Rossi, J.J. (2003). Approaches for the sequence-specific knockdown of mRNA. Nat. Biotechnol. 21, 1457-1465.

Schiwy, N.E. (2010). Axonale Regeneration und Plastizität unterschiedlicher Fasersysteme nach Rückenmarksverletzungen und verschiedenen Behandlungsansätzen in der adulten Ratte.

Schiwy, N., Brazda, N., and Müller, H.W. (2009). Enhanced regenerative axon growth of multiple fibre populations in traumatic spinal cord injury following scar-suppressing treatment. Eur. J. Neurosci. 30, 1544-1553.

Schnell, L., Fearn, S., Schwab, M.E., Perry, V.H., and Anthony, D.C. (1999). Cytokine-induced acute inflammation in the brain and spinal cord. J.

Neuropathol. Exp. Neurol. 58, 245-254.

Schratt, G.M., Tuebing, F., Nigh, E.A., Kane, C.G., Sabatini, M.E., Kiebler, M., and Greenberg, M.E. (2006). A brain-specific microRNA regulates dendritic spine development. Nature 439, 283-289. 
Schrock, Y., Solis, G.P., and Stuermer, C.A.O. (2009). Regulation of focal adhesion formation and filopodia extension by the cellular prion protein (PrPC). FEBS Lett. 583, 389-393.

Schulte, T., Paschke, K.A., Laessing, U., Lottspeich, F., and Stuermer, C.A. (1997). Reggie-1 and reggie-2, two cell surface proteins expressed by retinal ganglion cells during axon regeneration. Dev. Camb. Engl. 124, 577-587.

Schwab, M.E. (1996). Structural plasticity of the adult CNS. Negative control by neurite growth inhibitory signals. Int. J. Dev. Neurosci. Off. J. Int. Soc. Dev. Neurosci. 14, 379-385.

Schwartz, E.D., Cooper, E.T., Chin, C.-L., Wehrli, S., Tessler, A., and Hackney, D.B. (2005). Ex vivo Evaluation of ADC Values within Spinal Cord White Matter Tracts. Am. J. Neuroradiol. 26, 390-397.

Shearer, M.C., and Fawcett, J.W. (2001). The astrocyte/meningeal cell interface--a barrier to successful nerve regeneration? Cell Tissue Res. 305, 267-273.

Silva, N.A., Sousa, N., Reis, R.L., and Salgado, A.J. (2014). From basics to clinical: A comprehensive review on spinal cord injury. Prog. Neurobiol. 114, 25-57.

Solis, G.P., Schrock, Y., Hulsbusch, N., Wiechers, M., Plattner, H., and Stuermer, C.A.O. (2012). Reggies/flotillins regulate E-cadherin-mediated cell contact formation by affecting EGFR trafficking. Mol. Biol. Cell 23, 18121825.

Song, J., Takeda, M., and Morimoto, R.l. (2001). Bag1-Hsp70 mediates a physiological stress signalling pathway that regulates Raf-1/ERK and cell growth. Nat. Cell Biol. 3, 276-282.

Sroka, K., Voigt, A., Deeg, S., Reed, J.C., Schulz, J.B., Bähr, M., and Kermer, P. (2009). BAG1 modulates huntingtin toxicity, aggregation, degradation, and subcellular distribution. J. Neurochem. 111, 801-807.

Starkey, M.L., Bartus, K., Barritt, A.W., and Bradbury, E.J. (2012). Chondroitinase $\mathrm{ABC}$ promotes compensatory sprouting of the intact corticospinal tract and recovery of forelimb function following unilateral pyramidotomy in adult mice. Eur. J. Neurosci. 36, 3665-3678.

Steward, O., Zheng, B., and Tessier-Lavigne, M. (2003). False resurrections: distinguishing regenerated from spared axons in the injured central nervous system. J. Comp. Neurol. 459, 1-8.

Stichel, C.C., and Müller, H.W. (1994). Relationship between injury-induced astrogliosis, laminin expression and axonal sprouting in the adult rat brain. J. Neurocytol. 23, 615-630.

Stichel, C.C., Hermanns, S., Luhmann, H.J., Lausberg, F., Niermann, H., D'Urso, D., Servos, G., Hartwig, H.G., and Müller, H.W. (1999). Inhibition of 
collagen IV deposition promotes regeneration of injured CNS axons. Eur. J. Neurosci. 11, 632-646.

Stuermer, C.A.O. (2010). The reggie/flotillin connection to growth. Trends Cell Biol. 20, 6-13.

Sun, F., Park, K.K., Belin, S., Wang, D., Lu, T., Chen, G., Zhang, K., Yeung, C., Feng, G., Yankner, B.A., et al. (2011). Sustained axon regeneration induced by co-deletion of PTEN and SOCS3. Nature 480, 372-375.

Takayama, S., Sato, T., Krajewski, S., Kochel, K., Irie, S., Milian, J.A., and Reed, J.C. (1995). Cloning and functional analysis of BAG-1: A novel Bcl-2binding protein with anti-cell death activity. Cell 80, 279-284.

Tanaka, H., Yamashita, T., Yachi, K., Fujiwara, T., Yoshikawa, H., and Tohyama, M. (2004). Cytoplasmic p21(Cip1/WAF1) enhances axonal regeneration and functional recovery after spinal cord injury in rats. Neuroscience 127, 155-164.

Tator, C.H., and Fehlings, M.G. (1991). Review of the secondary injury theory of acute spinal cord trauma with emphasis on vascular mechanisms. J. Neurosurg. 75, 15-26.

Tator, C.H., and Koyanagi, I. (1997). Vascular mechanisms in the pathophysiology of human spinal cord injury. J. Neurosurg. 86, 483-492.

Taymans, J.-M., Vandenberghe, L.H., Haute, C.V.D., Thiry, I., Deroose, C.M., Mortelmans, L., Wilson, J.M., Debyser, Z., and Baekelandt, V. (2007). Comparative analysis of adeno-associated viral vector serotypes 1, 2, 5, 7, and 8 in mouse brain. Hum. Gene Ther. 18, 195-206.

Tester, N.J., and Howland, D.R. (2008). Chondroitinase ABC improves basic and skilled locomotion in spinal cord injured cats. Exp. Neurol. 209, 483-496.

Tetzlaff, W., Alexander, S.W., Miller, F.D., and Bisby, M.A. (1991). Response of facial and rubrospinal neurons to axotomy: changes in mRNA expression for cytoskeletal proteins and GAP-43. J. Neurosci. Off. J. Soc. Neurosci. 11, 2528-2544.

Thuret, S., Moon, L.D.F., and Gage, F.H. (2006). Therapeutic interventions after spinal cord injury. Nat. Rev. Neurosci. 7, 628-643.

Tönges, L., Koch, J.-C., Bähr, M., and Lingor, P. (2011). ROCKing Regeneration: Rho Kinase Inhibition as Molecular Target for Neurorestoration. Front. Mol. Neurosci. 4, 39.

Tönges, L., Frank, T., Tatenhorst, L., Saal, K.A., Koch, J.C., Szego, É.M., Bähr, M., Weishaupt, J.H., and Lingor, P. (2012). Inhibition of rho kinase enhances survival of dopaminergic neurons and attenuates axonal loss in a mouse model of Parkinson's disease. Brain J. Neurol. 135, 3355-3370.

Tönges, L., Günther, R., Suhr, M., Jansen, J., Balck, A., Saal, K.-A., Barski, E., Nientied, T., Götz, A.A., Koch, J.-C., et al. (2014). Rho kinase inhibition 
modulates microglia activation and improves survival in a model of amyotrophic lateral sclerosis. Glia 62, 217-232.

Tseng, G.F., Wang, Y.J., and Hu, M.E. (1996). Axotomy affects the retrograde labeling of cervical and lumbar-cord-projecting rubrospinal neurons differently. Anat. Embryol. (Berl.) 194, 457-464.

Ulusoy, A., Sahin, G., Björklund, T., Aebischer, P., and Kirik, D. (2009). Dose Optimization for Long-term rAAV-mediated RNA Interference in the Nigrostriatal Projection Neurons. Mol. Ther. 17, 1574-1584.

Vavrek, R., Girgis, J., Tetzlaff, W., Hiebert, G.W., and Fouad, K. (2006). BDNF promotes connections of corticospinal neurons onto spared descending interneurons in spinal cord injured rats. Brain 129, 1534-1545.

Verma, R.K., Arora, S., and Garg, S. (2004). Osmotic pumps in drug delivery. Crit. Rev. Ther. Drug Carrier Syst. 21, 477-520.

Wang, J.T., Medress, Z.A., and Barres, B.A. (2012). Axon degeneration: Molecular mechanisms of a self-destruction pathway. J. Cell Biol. 196, 7-18.

Wang, X., Baughman, K.W., Basso, D.M., and Strittmatter, S.M. (2006). Delayed Nogo Receptor Therapy Improves Recovery from Spinal Cord Contusion. Ann. Neurol. 60, 540-549.

Watzlawick, R., Sena, E.S., Dirnagl, U., Brommer, B., Kopp, M.A., Macleod, M.R., Howells, D.W., and Schwab, J.M. (2013). Effect and Reporting Bias of RhoA/ROCK-Blockade Intervention on Locomotor Recovery After Spinal Cord Injury: A Systematic Review and Meta-analysis. JAMA Neurol.

Webb, A.A., and Muir, G.D. (2003). Unilateral dorsal column and rubrospinal tract injuries affect overground locomotion in the unrestrained rat. Eur. J. Neurosci. 18, 412-422.

Weidner, N., Ner, A., Salimi, N., and Tuszynski, M.H. (2001). Spontaneous corticospinal axonal plasticity and functional recovery after adult central nervous system injury. Proc. Natl. Acad. Sci. U. S. A. 98, 3513-3518.

Wiessner, C., Bareyre, F.M., Allegrini, P.R., Mir, A.K., Frentzel, S., Zurini, M., Schnell, L., Oertle, T., and Schwab, M.E. (2003). Anti-Nogo-A antibody infusion 24 hours after experimental stroke improved behavioral outcome and corticospinal plasticity in normotensive and spontaneously hypertensive rats. J. Cereb. Blood Flow Metab. Off. J. Int. Soc. Cereb. Blood Flow Metab. 23, 154-165.

Wu, B., Bi, Z., and Qi, Q. (2013). Inactivation of the Rho-ROCK signaling pathway to promote neurologic recovery after spinal cord injuries in rats. Chin. Med. J. (Engl.) 126, 3723-3727.

Wu, D., Yang, P., Zhang, X., Luo, J., Haque, M.E., Yeh, J., Richardson, P.M., Zhang, Y., and Bo, X. (2009). Targeting a dominant negative rho kinase to neurons promotes axonal outgrowth and partial functional recovery after rat rubrospinal tract lesion. Mol. Ther. J. Am. Soc. Gene Ther. 17, 2020-2030. 
Wu, Z., Asokan, A., and Samulski, R.J. (2006). Adeno-associated virus serotypes: vector toolkit for human gene therapy. Mol. Ther. J. Am. Soc. Gene Ther. 14, 316-327.

Yezierski, R.P. (1996). Pain following spinal cord injury: the clinical problem and experimental studies. Pain 68, 185-194.

Yiu, G., and He, Z. (2006). Glial inhibition of CNS axon regeneration. Nat. Rev. Neurosci. 7, 617-627.

Zhang, X., Wang, H., Zhang, S., Song, J., Zhang, Y., Wei, X., and Feng, Z. (2012). MiR-134 functions as a regulator of cell proliferation, apoptosis, and migration involving lung septation. In Vitro Cell. Dev. Biol. Anim. 48, 131136.

Zhang, Y.P., Onifer, S.M., Burke, D.A., and Shields, C.B. (2001). A topical mixture for preventing, abolishing, and treating autophagia and self-mutilation in laboratory rats. Contemp. Top. Lab. Anim. Sci. Am. Assoc. Lab. Anim. Sci. 40, 35-36.

Zhao, R.-R., Andrews, M.R., Wang, D., Warren, P., Gullo, M., Schnell, L., Schwab, M.E., and Fawcett, J.W. (2013). Combination treatment with antiNogo-A and chondroitinase $A B C$ is more effective than single treatments at enhancing functional recovery after spinal cord injury. Eur. J. Neurosci. 38, 2946-2961.

Zolotukhin, I., Luo, D., Gorbatyuk, O., Hoffman, B., Warrington, K., Jr, Herzog, R., Harrison, J., and Cao, O. (2013). Improved Adeno-associated Viral Gene Transfer to Murine Glioma. J. Genet. Syndr. Gene Ther. 4.

Zolotukhin S et al. (1999). Recombinant adeno-associated virus purification using novel methods improves infectious titer and yield. Publ. Online 03 June 1999 Doi101038sjgt3300938 6. 


\section{Annexes}

\section{List of Abbreviations}

\begin{tabular}{|c|c|}
\hline$\sim$ & approximately \\
\hline $1 x, 2 x, 3 x$ & 1 time, 2 times, 3 times \\
\hline$\mu \mathrm{m}$ & micrometer \\
\hline AP & anterior-posterior \\
\hline AAD & acute axonal degeneration \\
\hline AAV & adeno associated virus \\
\hline $\mathrm{ABC}$ & avidin-biotin-complex \\
\hline ASB & antiscaring buffer treatment \\
\hline AST & antiscaring treatment \\
\hline ATP & adenosine triphosphate \\
\hline BAG1 & Bcl-2-associated athanogene-1 \\
\hline $\mathrm{BBB}$ & locomotor test/scale (developed by Basso, Beattie and \\
\hline \multicolumn{2}{|l|}{ Bresnahan) } \\
\hline BDA & biotinylated dextran amine \\
\hline BDNF & brain derived neurotrophic factors \\
\hline BPY-DCA & 2,2 - bipyridine-5,5`dicarboxylic acid (iron chelator) \\
\hline $\mathrm{CaCl}_{2}$ & calcium chloride \\
\hline cAMP & cycloadenosine monophosphate \\
\hline CNS & central nervous system \\
\hline CSF & cerebrospinal fluid \\
\hline CSPG & chondroitin sulfate proteoglycan \\
\hline CST & corticospinal tract \\
\hline CTB & cholera toxin B \\
\hline $\mathrm{CX}$ & cortex \\
\hline DAPI & 4‘,6-Diamidino-2-phenylindole \\
\hline DNA & deoxyribonucleic acid \\
\hline DsRed & Discosoma red fluorescent protein \\
\hline DV & dorsoventral \\
\hline CM & extracellular matrix \\
\hline & ED1 protein of lysosomes (CD68) \\
\hline
\end{tabular}




\begin{tabular}{|c|c|}
\hline Egf-R & epidermal growth factor receptor \\
\hline EGFP & enhanced green fluorescent protein \\
\hline ETOH & ethanol \\
\hline GFAP & glial fibrillary acidic protein \\
\hline$g$ & grams \\
\hline $\mathrm{H}_{2} \mathrm{O}_{2}$ & hydrogen peroxide \\
\hline $\mathrm{HCl}$ & hydrochloric acid \\
\hline hr. & hour \\
\hline hrs. & hours \\
\hline LHL & left hind limb \\
\hline MAG & myelin-associated glycoprotein \\
\hline $\mathrm{n}$ & number \\
\hline NOGO & myelin-associated inhibitors \\
\hline MAPK & mitogen-activated protein kinase \\
\hline $\mathrm{ML}$ & mediolateral \\
\hline miRNA & micro RNA \\
\hline $\mathrm{mm}$ & millimeter \\
\hline $\mathrm{Na}+$ & sodium \\
\hline $\mathrm{NaCl}$ & sodium chloride \\
\hline $\mathrm{NaOH}$ & sodium hydroxide solution \\
\hline $\mathrm{NgR}$ & NOGO receptor \\
\hline $\mathrm{NO}_{2}$ & nitrous oxide \\
\hline NSCs & neural stem cells \\
\hline $\mathrm{O}_{2}$ & oxygen \\
\hline OMGp & oligodendrocyte myelin glycoprotein \\
\hline $\mathrm{OMN}$ & occulo motor nerve fibers \\
\hline PBS & phosphate buffered saline \\
\hline PCR & polymerase chain reaction \\
\hline PFA & paraformaldehyde \\
\hline PNS & peripheral nervous system \\
\hline $\mathrm{RHL}$ & right hind limb \\
\hline $\mathrm{RN}$ & red nucleus \\
\hline RNA & ribonucleic acid \\
\hline ROCK & rho-associated kinase \\
\hline
\end{tabular}




$\begin{array}{ll}\text { rpm } & \text { rounds per minute } \\ \text { RST } & \text { rubrospinal tract } \\ \text { RT } & \text { room temperature } \\ \text { SC } & \text { spinal cord } \\ \text { SCI } & \text { spinal cord injury } \\ \text { shRNA } & \text { short hairpin RNA } \\ \text { SN } & \text { substantia nigra } \\ \text { Th } & \text { thoracic level } \\ \text { TRIS } & \text { TRIS(hydroxymethyl)-aminomethane } \\ \text { TU } & \text { transducing units } \\ \text { v/v } & \text { volume per volume } \\ \text { vh } & \text { ventral horn } \\ \text { vWF } & \text { von Willebrand factor, endothelial marker } \\ \text { w/v } & \text { weight per volume }\end{array}$




\section{Supplementary images}

a pAAV-9(5)-hSynEGFP-miR134

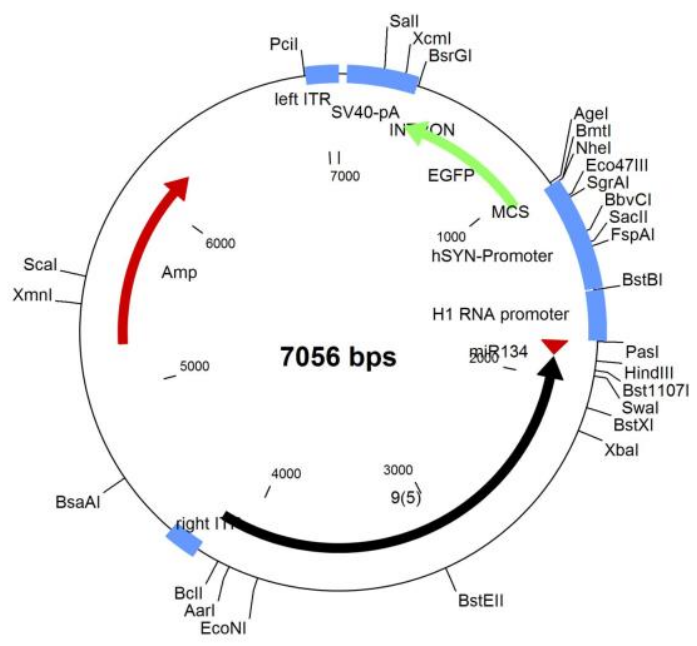

b

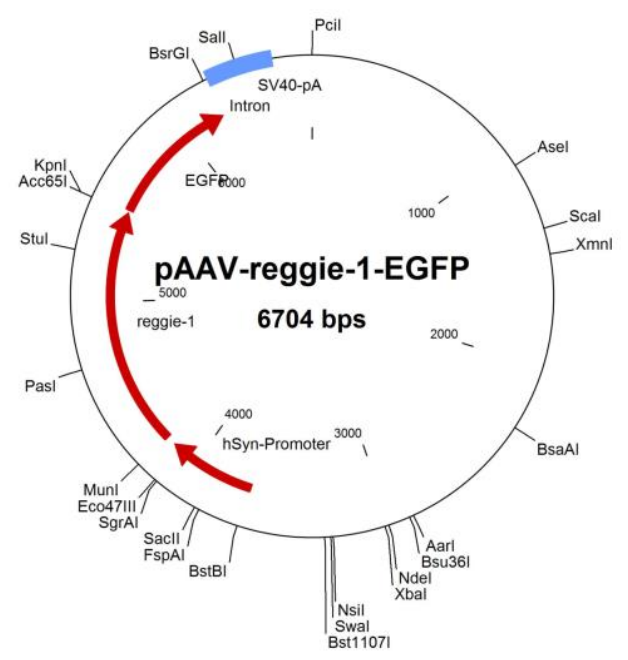

Vector maps of AAV.miRNA-134 and AAV.reggie1. Schematic depiction of vector maps of (a) AAV.miRNA-134, (b) AAV.reggie1. Restriction sites used for cloning were showed on the external side of the map. $\Pi R$, inverted terminal repeats; hSyn, human synapsin 1 gene promoter; Int, intron; SV40, simian virus 40 polyadenylation site; EGFP, enhanced green fluorescent protein reporter gene. Amp=ampicillin resistance gene. 
a

pAAV-9(5)-hSyn-EGFP-CytbAS-ohne Not

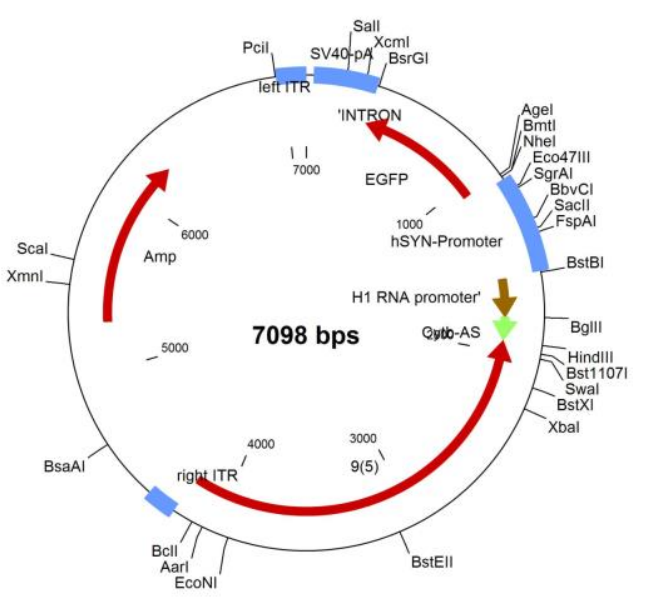

b

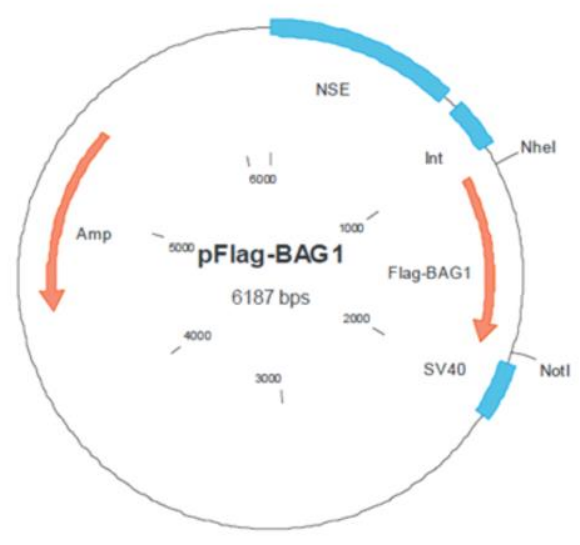

Vector maps of AAV.EGFP and AAV.BAG1 flag. Schematic depiction of vector maps of (a) AAV.EGFP, (b) AAV.BAG1 flag. Restriction sites used for cloning were showed on the external side of the map. ITR, inverted terminal repeats; hSyn, human synapsin 1 gene promoter; Int, intron; SV40, simian virus 40 polyadenylation site; EGFP, enhanced green fluorescent protein reporter gene. Amp=ampicillin resistance gene. 
a

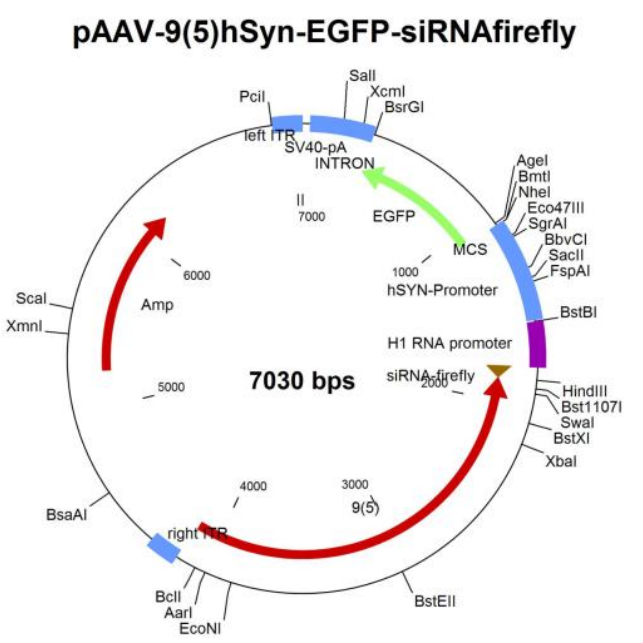

b

pAAV-9(5)-hSyn-EGFP-siRNA-Rock2

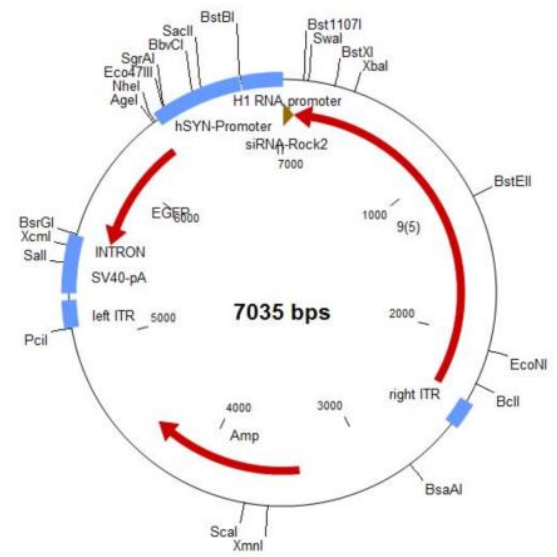

Vector maps of AAV.sh.firefly and AAV.sh.ROCK2. Schematic depiction of vector maps of (a) AAV.sh.firefly (b) AAV.sh.ROCK2, Restriction sites used for cloning were showed on the external side of the map. ITR, inverted terminal repeats; hSyn, human synapsin 1 gene promoter; Int, intron; SV40, simian virus 40 polyadenylation site; EGFP, enhanced green fluorescent protein reporter gene. Amp=ampicillin resistance gene. 


\section{Acknowledgements}

This work was carried out at the department of Neurology at the University of Medicine Göttingen (UMG), Göttingen. I would like to thank Prof. Mathias Bähr for giving me the opportunity to do my Ph.D. in his department and critical discussions, suggestions and supervision.

I would like to extend an enormous amount of gratitude to my close supervisor and mentor Prof. Dr. Paul Lingor. His dedicated guidance, encouragement, inspiration and emotional support were crucial for this thesis.

I would like to thank Prof. Dr. Uwe Michel, Dr. Jan Koch and Dr. Sebastian Kügler for providing the viral vectors for all my studies and fruitful discussions and suggestions.

I would like to acknowledge Prof Dr.Frank Bradke (University of Bonn) for helping in establishing the spinal cord lesion model and Prof Dr. Hans Werner Müller (University of Düsseldorf) group for implantation of osmoticmini pumps and help in establishing behavioral studies for the spinal cord injury paradigm.

I would like to thank specially Thomas Ostendorf for the friendly teaching of the spinal cord lesions and stereotactic brain injections and support for this project.

I would like to thank my thesis committee members Prof. Dr. Wolfgang Brück and Prof. Dr. André Fischer for their useful suggestions for my thesis.

I would like to thank my friends of our group: the girls of Paul's lab, Jianan, Kim, Anna, Eleonora and Zara; and the boys, Lars Tatenhost, Lars Toenges, Vinicius and David for their wonderful help in the daily struggle with words, deeds, fruitful discussions, encouragement and support. Special thanks goes to Lisa and Vivian for the excellent technical assistance, suggestions and 
special care in organizing events and birthday celebrations, which made me feel the lab as family and forget the distance apart from my family.

I would to thank all my fellow scientists of Waldweg, especially Yulia, Andrea, Johan, Kathrin, Monica, Anja for the fun times and interesting discussions.

I would like to thank all the Göttingen Telugu friends for the lovely times and celebrations.

A special thank goes to lovely couple Jan and Wang $Y_{i}$ for the wonderful dumplings, spicy dinners and outings, which helped me to refresh my mood and for the emotional support in needy times.

I would like to thank my best friends Narendra and Sahitya for everything and being my side all the time.

I want to thank my parents and brothers for their love and support. I would like to extend my love and gratitude to my elder brother Ajay whose encouragement, support and love was crucial throughout my education.

Last but not least I would like to thank my husband Sree for his patience and support and caring during my thesis. 
Annexes 